\title{
Spinal cord stimulation in neuropathic pain technical aspects and effectiveness
}

Citation for published version (APA):

Smits, H. (2011). Spinal cord stimulation in neuropathic pain technical aspects and effectiveness.

[Doctoral Thesis, Maastricht University]. Datawyse / Universitaire Pers Maastricht.

https://doi.org/10.26481/dis.20111219hs

Document status and date:

Published: 01/01/2011

DOI:

10.26481/dis.20111219hs

Document Version:

Publisher's PDF, also known as Version of record

\section{Please check the document version of this publication:}

- A submitted manuscript is the version of the article upon submission and before peer-review. There can be important differences between the submitted version and the official published version of record.

People interested in the research are advised to contact the author for the final version of the publication, or visit the DOI to the publisher's website.

- The final author version and the galley proof are versions of the publication after peer review.

- The final published version features the final layout of the paper including the volume, issue and page numbers.

Link to publication

\footnotetext{
General rights rights.

- You may freely distribute the URL identifying the publication in the public portal. please follow below link for the End User Agreement:

www.umlib.nl/taverne-license

Take down policy

If you believe that this document breaches copyright please contact us at:

repository@maastrichtuniversity.nl

providing details and we will investigate your claim.
}

Copyright and moral rights for the publications made accessible in the public portal are retained by the authors and/or other copyright owners and it is a condition of accessing publications that users recognise and abide by the legal requirements associated with these

- Users may download and print one copy of any publication from the public portal for the purpose of private study or research.

- You may not further distribute the material or use it for any profit-making activity or commercial gain

If the publication is distributed under the terms of Article $25 \mathrm{fa}$ of the Dutch Copyright Act, indicated by the "Taverne" license above, 


\section{Spinal cord stimulation in neuropathic pain technical aspects and effectiveness}

$$
\text { Helwin Smits }
$$


We are indebted to Medtronic Europe SA for financial research support. The Bakken Research Center in Maastricht deserves gratitude for supplying the electrodes (special regards to Paul van Venrooij and Victor Duysens).

The studies within this thesis were performed within TREND (Trauma Related Neuronal Dysfunction), a knowledge consortium that integrates research on Complex Regional Pain Syndrome type 1, and is supported by a Dutch government grant (BSIK03016).

(C) Copyright Helwin Smits, Maastricht 2011

ISBN 9789461591081

Production: Datawyse | Universitaire Pers Maastricht 


\title{
Spinal cord stimulation in neuropathic pain technical aspects and effectiveness
}

\author{
PROEFSCHRIFT \\ ter verkrijging van de graad van doctor aan de Universiteit Maastricht, \\ op gezag van de Rector Magnificus, Prof. mr. G.P.M.F. Mols, \\ volgens het besluit van het College van Decanen, \\ in het openbaar te verdedigen, \\ op maandag 19 december 2011 om 12:00 uur \\ door
}

Helwin Smits

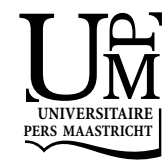




\section{Promotor}

Prof. dr. M. van Kleef

\section{Co-promotores}

Dr. E.A.J. Joosten

Dr. M.A. Kemler

\section{Beoordelingscommissie}

Prof. dr. V. Visser-Vandewalle, voorzitter

Prof. dr. M. de Baets

Prof. dr. J. Holsheimer, Universiteit Twente

Prof. dr. F.J.P.M. Huygen, Erasmus MC Rotterdam

Prof. dr. H. Vles 


\section{Contents}

Abbreviations

$\begin{array}{lll}\text { Chapter } 1 & \text { Introduction } & 7\end{array}$

Chapter 2 Experimental Spinal Cord Stimulation and Neuropathic Pain: 21 mechanism of action, technical aspects and effectiveness

Chapter 3 Effect of Spinal Cord Stimulation in an Animal Model of Neuropathic Pain Relates to Degree of Tactile "Allodynia"

Chapter 4 Spinal Cord Stimulation of dorsal columns in a rat model of neuropathic pain: evidence for a segmental spinal mechanism of pain relief

Chapter 5 Spinal Cord Stimulation induces c-Fos expression in the Dorsal Horn in Rats with Neuropathic Pain after Partial Sciatic Nerve Injury

Chapter 6 Spinal Cord Stimulation for Complex Regional Pain Syndrome type-1: A cohort study with twelve year follow up

Chapter 7 Summary and general discussion

References

Slotoverweging en dankwoord

Curriculum vitae 


\section{Abbreviations}

$\begin{array}{ll}\text { AF } & \text { Activation Function } \\ \text { AMPA } & \text { a-amino-3-hydroxy-5-methyl-4-isoxazolepropionic acid } \\ \text { ANOVA } & \text { Analysis of variance } \\ \text { BDNF } & \text { Brain-Derived Neurotrophic Factor } \\ \text { CCI } & \text { Chronic Constriction Injury } \\ \text { CNS } & \text { Central Nervous System } \\ \text { CRPS } & \text { Complex Regional Pain Syndrome } \\ \text { DPO } & \text { Days Post Operative } \\ \text { DR } & \text { Dorsal Root } \\ \text { DH } & \text { Dorsal Horn } \\ \text { DRG } & \text { Dorsal Root Ganglion } \\ \text { EAA } & \text { Excitatory Amino Acid } \\ \text { FBSS } & \text { Failed Back Surgery Syndrome } \\ \text { GABA } & \text { Gamma-Amino Butyric Acid } \\ \text { Glu } & \text { Glutamate } \\ \text { HRQol } & \text { Health related quality of life } \\ \text { Hz: } & \text { Hertz } \\ \text { i.t. } & \text { intrathecal } \\ \text { IASP } & \text { International Association for the Study of Pain } \\ \text { LTD } & \text { Long Term Depression } \\ \text { LTP } & \text { Long Term Potentiation } \\ \text { MPE } & \text { Maximum Possible Effect } \\ \text { MT: } & \text { Motor Threshold } \\ \text { NMDA } & \text { N-methyl D-aspartate } \\ \text { NPP } & \text { Neuropathic pain } \\ \text { NS } & \text { Nociceptive Specific } \\ \text { PIPWTH } & \text { Pre-Injury Paw Withdrawal Thresholds } \\ \text { PWT } & \text { Paw Withdrawal Threshold } \\ \text { RCT: } & \text { Randomized Clinical Trial } \\ \text { SCS } & \text { Spinal Cord Stimulation } \\ \text { SCS: } & \text { Spinal Cord Stimulation } \\ \text { SD } & \text { Sprague-Dawley } \\ \text { SEM: } & \text { Standard Error of the Mean } \\ \text { SP } & \text { Substance P } \\ \text { SSNRI } & \text { Serotonin-Norepinephrine Reuptake Inhibitor } \\ \text { TCA } & \text { Tricyclic Antidepressants } \\ \text { TNF } & \text { Tumor Necrosis Factor } \\ \text { VAS: } & \text { Visual Analogue Scale } \\ \text { WDR } & \text { Wide Dynamic Range } \\ & \end{array}$


Chapter 1

Introduction

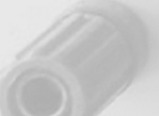

N-

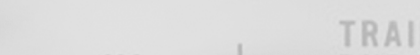

MS

DURATION

TRAIN DUR.

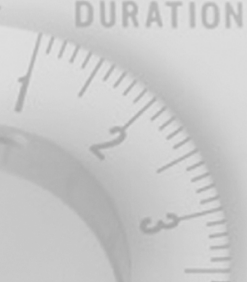

$\mathrm{S}_{2}$

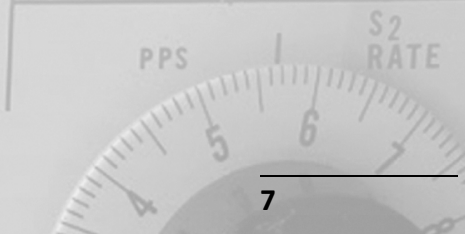





\section{Neuropathic pain: the problem}

"In our early experience of nerve wounds, we met with a small number of men who were suffering from a pain which they described as 'burning,' as 'mustard red hot' or as a 'red hot file rasping the skin.' Its intensity varies from the most trivial burning to a state of torture."

Dr. S.W. Mitchell (1829-1914) Union army physician during the American civil war

The above symptoms presented by injured soldiers during the American civil war clearly describe neuropathic pain, long before any scientific theories or rational treatments of Neuropathic pain (NPP) existed. NPP was defined as pain initiated or caused by a primary lesion or dysfunction in the nervous system in 1994 (2). This definition of NPP however lacks both anatomic specificity and precision and therefore the Neuropathic Pain Special Interest Group (NeuPSIG) reformulated NPP in 2009 as: "Pain arising as a direct consequence of a lesion or disease affecting the somatosensory system either at peripheral or central level"(3). The concept is that in NPP somatosensory processing is aberrant and goes beyond the normal plasticity of the nociceptive system. Both human and animal research indicate that for the development of NPP a lesion of afferent pathways is necessary (4). The actual nerve damage causing NPP can have different aetiologies, for example: infection, trauma, surgery, metabolic disturbances, radiation, chemotherapy, neurotoxins, compression, and inflammation or tumor infiltration. Based on either a peripheral or central anatomical location of a lesion or disease neuropathic pain can be classified as either peripheral or central NPP. It is likely however that in many cases NPP involves both peripheral and central mechanisms. As to the symptoms and signs, NPP presents either as spontaneous pain or as stimulus-evoked pain. Spontaneous pain is present in the absence of any stimulation; it can have a continuous or more intermittent nature and does often have a shooting, stabbing or electrical character. Stimulus-evoked pain consists of allodynia; pain in response to a normally non painful stimulus and hyperalgesia; increased pain in response to a normally painful stimulus. The rule rather than the exception is that most patients with NPP have more than one type of pain (5).

Recent studies indicate that NPP causes a significant decrease in patients health related quality of life (HRQol), including emotional and physical functioning and is associated with substantial societal costs $(6,7)$. The incidence rate of neuropathic pain is 8.2 per 1000 person years (8) and is expected to increase in the near future as the population is aging and NPP is more common in the elderly. The management of NPP should always be placed in context of the underlying disorder (e.g. CRPS, diabetic neuropathy). Treatment of NPP can be challenging as it is often refractory to existing treatments. 


\section{Complex Regional Pain Syndrome (CRPS)}

Complex Regional Pain Syndrome is a neuropathic pain disorder which is most commonly initiated by surgery, fractures, crush injuries or sprains but can also develop spontaneously (9) CRPS is a chronic neuropathic pain disorder that requires early referral to a specialist pain clinic and typically develops in an extremity after tissue trauma. The affected area usually extends beyond the original injury often with a glove- or sock-like distribution. CRPS is characterized by the following symptoms; continuous pain, sensory dysfunction (hyperalgesia, allodynia, hypoalgesia and mechanical hypoesthesia) vasomotor dysfunction (color difference), sudomotor dysfunction (local oedema), motor- (loss of strength, decreased active range of motion and tremor and trophic signs (skin, hair and nail growth changes). CRPS can be subdivided in CRPS-1 and CRPS- 2 reflecting the documented absence or presence of nerve injury respectively (2). The pathophysiology of CRPS-1 is complex and is still under research. In the current perspective multiple mechanisms may be involved that comprise of the following: Changes in cutaneous innervation with a reduced density of $C$ and $A \delta$ fibres in the affected region, central and peripheral sensitization, altered sympathetic nervous system function and local changes in circulating catecholamines, inflammatory responses with an increased local, systemic and cerebrospinal fluid levels of pro-inflammatory cytokines (TNF, and interleukine $1 \beta$, 2 and -6$)$, changes in brain plasticity, genetic factors and psychological factors (1016). A recent study by de Boer et al (17) demonstrated that the signs and symptoms of CRPS-1 differed with the duration of the disease and furthermore reported that the prevalence of allodynia and hyperalgesia was significantly higher in patients with a longer disease duration. In contrast the prevalence of color difference (vasomotor signs) and edema (sudomotor signs) is decreased with a longer duration of disease (17). The treatment of CRPS is challenging as there is no definitive effective treatment up till now (18). Hence invasive and expensive options like SCS are often used in CRPS, especially in CRPS-1 patients who fail to improve with medication, physical therapy or less invasive procedures and who thus require more aggressive or additional pain therapy (19).

\section{Mechanism underlying neuropathic pain}

\section{Peripheral sensitization}

In peripheral sensitization the peripheral nociceptive nerve terminals become hypersensitive after tissue injury and will be driven harder for any given stimulus due to inflammatory changes induced by mechanical, chemical or thermal tissue damage. After tissue injury many inflammatory mediators for example prostaglandin E2, bradykinin, substance $P$ and nerve growth factor are released (20). These mediators 
act on G-protein and tyrosine kinase receptors which are located on nociceptor cells activating intracellular signalling pathways causing a phosphorylation of ion channels and receptors in the nociceptor cell membrane, for example the sensoryneuron specific voltage gated sodium channel $\left(\mathrm{Na}_{v} 1.8\right)$ and the transient receptor potential V1 (TRVP-1 receptor) eventually increasing the excitability and sensitivity of the nociceptor in the region of inflammation (21-23).

\section{Central sensitization}

In 1965 Melzack and Wall introduced the Gate Control Theory (24) an important new insight in (spinal) pain processing with two important characteristics:

1. All sensory information from peripheral thin unmyelinated $\mathrm{C}$-fibres and thicker myelinated $A \beta$-fibres converges onto a dorsal horn $(\mathrm{DH})$ spinal network of excitatory - and inhibitory interneurons.

2. In this DH spinal network modulation of the signal takes place and a processed signal is finally send through to the second order Wide Dynamic Range (WDR) neuron from where it is passed to higher centres in the brain.

After peripheral sensitization has been established ongoing peripheral sensory activity induces central changes (central sensitization) in the DH spinal network. The central terminals of the primary nociceptive afferents release the excitatory neurotransmitter glutamate and often at the same time and same terminal the neurotransmitter substance $P$ as well as the neurotrophic factor Brain Derived Neurotrophic Factor (BDNF) (25). Glutamate activates ionotropic (NMDA, AMPA and kainate) as well as metabotrophic (mGlu1 and mGlu2) receptors which are located on the postsynaptic membrane. Abundant preclinical evidence indicates that the activation of the NMDA receptor mainly through phosphorylation of various subunits, in particular the NR2B subunit, is crucial to pain hypersensitivity (26);(27);(28). This pain hypersensitivity or central sensitization is therefore mainly related to the activation of the NMDA receptor in the DH spinal network (29). In this respect it is important to note that also BDNF, which is released at the same glutamatergic presynaptic terminal modulates the phosporylation of the NMDA receptor via the protein tyrosine kinase Src (30). The phosphorylation of the NMDA-NR subunits results in an increase $\mathrm{Ca}^{2+}$ influx. Increased levels of $\mathrm{Ca}^{2+}$ in the cytosol furthermore results in an increased synaptic activity through: a. the phosphorylation of the NMDA-NR subunits, b. trafficking of new AMPA-receptors to the postsynaptic membrane $(29,31)$ and c. an increased expression of voltage gated sodium channels (32).

This process of central sensitization causes amplification and prolongation of the incoming sensory stimuli which would normally be strongly reduced by the inhibitory GABA-ergic glycinergic and peptidergic interneurons as well as supraspinal descending modulation (33). The pivotal role of GABAergic inhibitory interneurons located in spinal laminae 1-3 (34) interfering with nociceptive activation of 
pain-signalling neurons has already been pointed out decennia ago (24). In line with this, pharmacological data have clearly confirmed that loss of endogenous GABAergic synaptic activity by blocking presynaptic metabotropic $\mathrm{GABA}_{B}$ receptors or postsynaptic ionotropic $\mathrm{GABA}_{A}$ leads to tactile and thermal hypersensitivity $(35,36)$ by enhancing excitatory neurotransmitter release from primary afferents or impairing hyperpolarization of spinal pain transmission neurons, respectively (37). In a recent study from our laboratory a differential role of GABA in development and maintenance of NPP was demonstrated: a dysfunctional GABA production is likely to be involved in early NPP whereas late NPP is characterized by a combined dysfunctional GABA release and decreased $K C C 2$ levels, the latter suggesting an impaired $\mathrm{GABA}_{A}$ receptor-mediated inhibition (38).

In summary: the induced postsynaptic glutamatergic changes combined with a changed role of the inhibitory and modulating GABA-ergic cells in the DH spinal cord enable low threshold mechanosensitive $A \beta$ and $A \delta$ fibres to activate second order nociceptive neurons (WDR) causing allodynia and hyperalgesia. Finally, although neuronal components and neurotransmission definitely are crucial in development and maintenance of NPP the role of glial cells is moving more and more to the forefront of scientific research in understanding NPP(39). At the moment a crucial role of multi-potent microglial cells in the initiation of central sensitization is suggested whereas the astroglial cell is thought to be mainly involved in the maintenance of NPP (40)

\section{Treatment of NPP}

The treatment of neuropathic pain is challenging as it is a disorder with multiple aetiologies, symptoms and underlying mechanisms. Up till now there is still no single successful treatment to prevent or cure neuropathic pain and at present the primary goal of treatment is the reduction of pain. For the initial treatment of NPP there are non-pharmacological and pharmacological options. There is some evidence for Physical Therapy as a non pharmacological treatment option for NPP, especially in CRPS patients $(41,42)$. The Neuropathic Pain Special Interest Group (NeuPSIG) developed an evidence based guideline for the first, second and third-line pharmacological treatment of NPP by combining evidence from randomized controlled trials with expert opinion (43). The mainstay of medications that are used in the first line treatment of neuropathic pain can be categorized as anticonvulsants and antidepressants. The antidepressants such as tricyclic antidepressants (TCA's) are often used in combination with serotonin and norepinephrine re-uptake inhibitors (SSNRI's). Anticonvulsants, basically ligands to the Calcium channel $\alpha 2-\delta$ subunit (gabapentin and pregabalin) are also used as first line treatment in NPP. In addition to TCA's or SSRNI's lidocaine 5\% medicated plaster are used as a first line 
treatment for NPP. Second line treatments, like opioids and tramadol, are used in patients unresponsive to first line.

Then finally, from a pharmacotherapeutic point of view various specialist or third line interventions currently are used:

Capsaicin: licensed for NPP and is used in post-herpetic neuralgia, nerve injury and mixed neuropathic pain conditions, or

- Second-line anticonvulsant drugs (unlicensed for neuropathic pain)e.g. lamotrigine, sodium valproate, clonazepam, or

- Ketamine an NMDA receptor antagonist, or

- Cannabinoids, which act on central and peripheral cannabinoid receptors, or

- Strong opioids: morphine, oxycodone and transdermal fentanyl and buprenorphine, or

- Lidocaine by intravenous infusion, or

- Selective serotonin re-uptake inhibitors and duloxetine.

In spite of the current multitude of pharmacological options many patients are refractory to the best therapies available or experience severe side effects (44).

\section{Interventional treatments for NPP}

Interventional treatments for NPP is reserved for patients whose pain is not adequately treated with first-, second-, and third-line pharmacotherapy or for patients with side-effects from medications. Interventional treatments should be carried out as part of a multidisciplinary treatment plan. A variety of interventional NPP treatments are currently being used including:

- Steroid injection; peripherally or centrally (dorsal root ganglion, epidural space)

- Neuromodulation or the stimulation or inhibition of neural pathways, as there are: Spinal cord stimulation (SCS) or (Pulsed) Radio Frequency (P)RF

- Blockade of the sympathetic nervous system in the cervical, thoracic and lumbar regions with local anaesthetics, through the use of Radiofrequency Lesioning or by nerve destruction.

In this thesis the (neuro)modulation of NPP by means of spinal cord stimulation (SCS) is the main subject of research.

\section{Neuromodulation for NPP}

The ability to generate, store and control electricity eventually led to the application of therapeutic electrical stimulation of nerve tissue or neuromodulation. Neuromodulation for the relief of NPP came into clinical practice during the late nineteen sixties and demonstrated to be a mainly non-destructive technique with reversible effects. Modern day neuromodulatory techniques used in the clinic for the relief of 
NPP consist of: Spinal Cord Stimulation (SCS) especially for complex regional pain syndrome type-1 (CRPS-1) and failed back surgery syndrome (FBSS) (45), transcutaneous electro nerve stimulation (TENS), motor cortex stimulation and (Pulsed) Radio Frequency (P)RF stimulation of the dorsal root ganglia.

\section{Spinal cord stimulation (SCS) for NPP: Clinic}

The clinical use of SCS was first reported by Shealy et al. two years after the introduction of the classical Gate-Control theory by Melzack and Wall $(24,46)$. Although SCS was tried for chronic and acute nociceptive pain, it appeared to have a pain relieving effect in NPP, especially in CRPS-1 and Failed Back Surgery Syndrome (FBSS) $(47,48)$. Although the Gate Control theory provided an initial basic concept, the detailed mechanism of action is not entirely clear. SCS is considered an end line pain therapy for NPP. Complex Regional Pain Syndrome type-1 (CRPS-1) and the Failed Back Surgery Syndrome (FBSS) are the only indications for SCS with a grade 3 level of evidence of a pain relieving effect (45). For CRPS-1 SCS is successful in nearly $60 \%$ of well selected patients as defined by at least $50 \%$ pain relief (a mean $25 \mathrm{~mm}$ reduction in Visual Analogue Score (VAS) score) (47) As to the clinical effectiveness of SCS as a pain therapy for CRPS-1 it may be concluded that SCS therapy has the following limitations as related to the pain relieving effect in itself:

1. SCS has a $40 \%$ non response rate (or "patients who do not reach a $50 \%$ pain reduction")

2. Although nearly $60 \%$ of patients (responders) do have a $50 \%$ reduction in pain they still suffer from light to moderate pain based on their VAS (4-5) (49), and furthermore a limitation based on the duration of the pain relieving effect of SCS:

3. The duration of the pain relieving effect of SCS is only short and ceases soon after SCS is attenuated.

Hence, important present day clinical issues are the improvement of the effectiveness of SCS in the form of enhancement of the pain relieving effect and enhancement of the duration of the pain relieving effect of SCS. With respect to the enhancement of the pain relieving effect it is a major goal first to turn non-responders to SCS into responders and second to provide patients who already respond to SCS to provide a better pain relief as most patients still suffer from moderate pain even with SCS. Furthermore, it needs no further comment that if the duration of the pain relieving effect can be increased this will have important implications for use of the SCS system.

Another major drawback of SCS as a pain therapy is that the non response to SCS, which occurs in $40 \%$ of patients, cannot be reliably predicted. In clinical SCS the general approach is to start with a test stimulation period of about one week with an external SCS device. Only when the test stimulation is successful (in around 60\% 
of patients) a permanent device will be implanted and this exposes the patient to two invasive procedures with common complications for example displacement of the electrode, requiring surgical re-intervention. The complication rate is $31-38 \%$ within the first two years of stimulation (50). The current practise of SCS as a pain therapy exposes the patient to an invasive therapy with a fairly high cost and complication rate and stresses the need to search for prognostic factors that may predict a successful outcome of SCS.

\section{Spinal cord stimulation (SCS) for NPP: experimental}

In 1994 a rat model of SCS in neuropathic pain was developed which allowed to investigate the mechanisms of action of SCS with a focus on the electrophysiological - and biochemical (neurotransmitter) changes of SCS induced pain relief in the dorsal horn (reviewed in chapter 2) (51-55) A frequently used experimental model for studies on SCS in NPP is the partial sciatic nerve ligation model (PSNL) as described by Seltzer et al in 1990 (56). In this model NPP is induced in (Spraque Dawley) rats through a partial (1/3-1/2) ligation of the sciatic nerve at a high thigh level. This ligation induces mechanical allodynia, heat evoked hyperalgesia and spontaneous pain within a few hours after the ligation which are present for as long as seven months (57). Whereas the PSNL model suffers with a large variability in behavioural outcome (see also Chapter 2 ) we aimed at improving the reproducibility of the Seltzer model. In our studies the sciatic nerve ligation was standardized by the use of clear anatomic landmarks at a level where the sciatic nerve is still monofascicular (see Chapter 3).

After development of NPP, as verified by reduced withdrawal thresholds assessed with the von Frey method, neuromodulation or SCS of the ascending fibre located in the dorsal columns is used. Two weeks after the nerve ligation a monopolar SCS system is implanted by means of a small laminectomy at a T13 vertebral level. A small plate electrode (cathode) (see fig.1) into the epidural space. The electrode is immobilized in the epidural space by a tight fixation of the wire to the adjacent spinous process with hysto-acryl tissue glue. A circular anode plate electrode is placed subcutaneously. After this the animal is allowed to recover from the implantation until SCS is performed at day 16 . In order to increase the effectivity and reproducibility of the SCS neuromodulation approach in this rat model we introduced into our studies a systematic control for localization of the electrodes by X-ray (see Chapter 3,4). The animal is connected to a constant current (Grass) stimulator and to ascertain that the SCS system is functional the minimal Motor Threshold (MT) is determined (specific muscle contractions). Then SCS intensity is adjusted to a certain percentage of MT (often 66\%) and stimulation is started. During SCS the level of allodynia was tested using the withdrawal response to tactile stimuli with the von Frey test, at regular intervals. From the results it appeared that the PSNL-SCS rat 
model has a high degree of clinical relevance as a number of experimental findings on the mechanisms of SCS appeared to be translational into the clinical practice. Important translational issues that up till now are addressed in the rat PSNL-SCS model were:

1. enhancement of the pain relieving effect of SCS and the reduction of nonresponders to SCS, and

2. enhancement of the duration of the effect of SCS

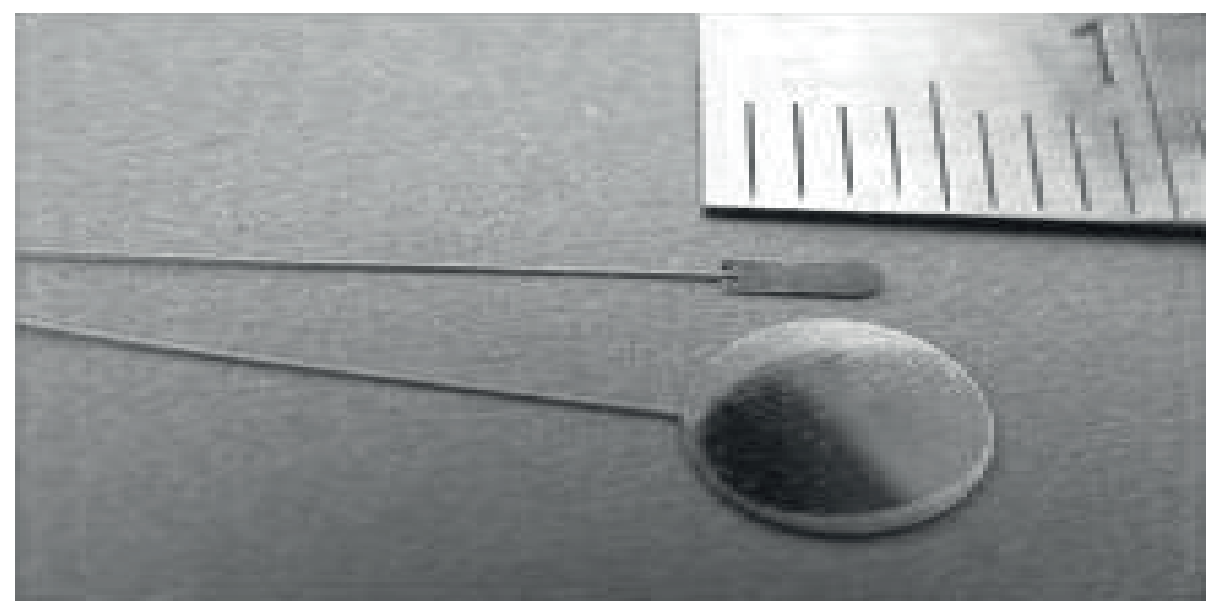

Figure 1: Platina-iridium experimental SCS system Anode: length $3.00 \mathrm{~mm}$, width $1.00 \mathrm{~mm}$ and thickness $0.100 \mathrm{~mm}$. Cathode circular diam. $6 \mathrm{~mm}$, thickness $0.100 \mathrm{~mm}$

1. Enhancement of the pain relieving effect of SCS and the reduction of nonresponders to SCS by means of

a. Pharmacological enhancement of the SCS-effect. The concept of pharmacological enhancement of SCS was first reported by Cui et al (58). Rats with nerve injury induced NPP that did previously not respond to SCS with a decrease in withdrawal thresholds could be turned into responders with the intrathecal administration of the $\mathrm{GABA}_{\mathrm{B}}$ agonist baclofen. These experimental results were confirmed clinically by Lind et al; (59) It should be noted, however, that the enhancement of the pain relieving effects of SCS by intrathecal administration with a baclofen pump were only studied in a very small $(n=7)$ number of patients and thus these very promising results should be interpreted with caution. Another example of pharmacological enhancement of the pain relieving effect of SCS was reported by Song et al (60). In this study a low dose of intrathecal serotonin markedly enhanced the pain relieving effect on tactile allodynia and cold hypersensitivity. A potent anti-nociceptive effect of intrathecally administered serotonin was previously described in diabetic and neuropathic rats $(61,62)$. The analgesic ac- 
tion of serotonin in neuropathic pain is at least partially mediated via spinal GABA-ergic and cholinergic mechanisms and involves different subtypes of $5-\mathrm{HT}$ receptors which are also involved in the pain relieving effect of SCS $(58,63,64)$. The serotonergic enhancement of SCS may act either through descending inhibition by SCS induced activation of higher centres in the brain or through direct activation of fibres in the Dorsolateral Funiculus lateral to the dorsal columns $(60,65)$.

Besides the modulation of either the GABA-ergic system or through interfering with the descending serotonergic input on the Pain Gate in the spinal dorsal horn a direct pharmacological modulation of the process of central sensitization of the glutamatergic synapses may also enhance the SCS effect. It now has been experimentally shown that non-responders to SCS can be turned into responders by the blocking of the NMDA receptor through the use of the non-competitive blocker ketamine (66). Although not yet clinically studied these experimental results provide hope for successful use of NDMA blockers in treatment of NPP patients who do not respond to SCS.

b. Early spinal cord stimulation. In a recent rat experimental study Truin et al (67) it was noted that early timing of SCS at 24 hours after nerve injury versus 16 days after nerve injury is more effective as compared to late SCS treatment. Out of 13 allodynic rats that received early SCS 10 (or 77\%) responded to SCS with significantly increased withdrawal thresholds, compared to $38 \%$ in the late SCS group (67). This may indicate that NPP presents a window of opportunity for more effective pain relief with the use of SCS early on in the disease (67). Up till now, however, the clinical practice with respect to use of SCS has evolved itself into an end stage therapy of intractable NPP. (This may be the result of the fact that the diagnosis of NPP is often delayed) A first attempt to study the effect of early SCS in a clinical situation was undertaken by van Eijs et al (68). Here, SCS was performed in a small number $(n=6)$ of early CRPS-1 patients. As different from the experimental situation these early CRPS-1 patients were characterized by a disease duration less than one year. The results of the study showed no clear benefit in pain relief of SCS in early CRPS-1 versus SCS in chronic CRPS-1 patients. A critical note needs to be mentioned here: in the early SCS experimental study treatment was performed within 24 hours after the nerve injury. Central sensitization which is a key event during the development of NPP is known to develop within days or hours (29). At an early stage the process of central sensitization is characterized by reversible and short term changes (29) and perhaps the enhancement of the pain relieving effects of SCS should be looked after within this very early stage. 


\section{Enhancement of the duration of the effect of SCS}

In experimental as well as in clinical SCS the duration of the pain relieving effects in NPP was relatively short once the stimulator is turned off. In an experimental study where SCS is applied 24 hours after the induction of neuropathic pain a significant increase in the duration of the increase of withdrawal thresholds was noted (67): An increase of the withdrawal threshold in the early SCS group could still be noticed 90 minutes after termination of SCS and in more than half of these animals, pre-stimulation withdrawal thresholds were reached only the next day (67). These results suggest that timing of the SCS intervention related to the development of NPP is probably important for the duration of its pain relieving effect. The success of early timing of SCS in treatment of NPP may be related to an early intervention in development of central sensitization which takes only days and hours to develop, and which is still reversible to some extend at the early stages (29).

\section{Aims, research questions and outline of this thesis}

As SCS has emerged as a last resort effective pain therapy in NPP states that are very resistant to the conventional treatments. Still many clinical questions regarding the effectiveness of the SCS therapy in NPP are unanswered. Clearly further investigation into the underlying mechanisms based on experimental work may give answers to the clinical questions. In our studies we focussed on the effectiveness in view of mechanism and predictability of the pain relieving effect of SCS. Whereas one of the main questions with respect to (clinical) use of SCS in treatment of NPP is how to enhance the effectiveness of this therapy we formulated the following research questions and outline of this thesis:

1. What is the current status and effectiveness of SCS in the treatment of NPP as related to its technical and anatomical aspects?

2. Are there signs or symptoms of neuropathic pain that may predict the outcome of SCS in terms of pain relief?

3. Does SCS of the dorsal columns act via a segmental spinal mechanism and is this important for the effectiveness of the therapy?

4. What is the long term (twelve year follow-up) effectiveness of SCS in CRPS-1 patients in terms of pain relief and health related quality of life. (HRQol)

The first question is addressed in Chapter $\mathbf{2}$ where the current understanding of the technical aspects of SCS is reviewed with recommendations for technical improvements that may enhance the effectiveness of SCS. The search for a possible predictor of success of SCS in treatment of NPP is the main aim of the experimental study described in Chapter 3. More specific: the effect of SCS in an animal model of neuropathic pain is studied in relation to the degree of tactile "allodynia". In Chapters 4 
and 5 the underlying mechanism of SCS induced pain relief is studied. As from a conceptual point of view but also related to the effectiveness of the therapy is important to know if the mechanism of SCS induced pain relief is based on a segmental spinal or a supraspinal mode of action (Chapter 4). In Chapter 5 the activity of cells after SCS treatment in experimental model of NPP was studied based on the expression of the immediate early gene c-Fos.

The effectiveness of pain relief of SCS in treatment of CRPS type 1 patients is described in Chapter 6: Spinal Cord Stimulation for Complex Regional Pain Syndrome type-1: A cohort study with up to twelve years of follow up. 

Chapter 2

Experimental Spinal Cord Stimulation and Neuropathic Pain: mechanism of action, technical aspects and effectiveness

H.Smits, M. van Kleef, J. Holsheimer, E.A.Joosten. 


\begin{abstract}
Spinal cord stimulation (SCS) a is valuable treatment for chronic intractable neuropathic pain. Although SCS has gone through a technological revolution over the last four decades the neurophysiologic- and biochemical mechanisms of action have only been partly elucidated. Animal experimental work has provided some evidence for spinal as well as supraspinal mechanisms of neuropathic pain relief of SCS. A SCS computer model of the electrical properties of the human spinal cord revealed many basic neurophysiologic principles which were clinically validated later on. The main question in clinical SCS is how to further improve the effectiveness of SCS, especially as related to the still significant failure rate of $30 \%$. In this context experimental studies are needed to elucidate which target pain neuron(s) are involved as well as with what exact electrical stimulation this target neuron can be influenced to produce an optimal suppression of neuropathic pain. This article reviews the basic clinical and experimental technical aspects in relation to the effectiveness of SCS in view of recent understanding of the dorsal horn pain circuit involved. These data may then result in experiments needed for an improved understanding of the mechanisms underlying SCS and consequently lead to improvement and increased effectiveness of SCS in neuropathic pain as a clinical therapy.
\end{abstract}




\section{Introduction}

\subsection{Spinal Cord Stimulation (SCS)}

Clinical use of electrical stimulation of the spinal cord was first reported by Shealy et al in 1967 and was a direct result of the new insight in pain and pain modulation provided by the Gate Control theory of Melzack and Wall (24) two years earlier. Today Spinal Cord Stimulation (SCS) is used in the treatment of intractable neuropathic pain in CRPS-1 as well as in a variety of other neuropathic pain conditions. Despite the existence of SCS as a pain therapy for over 40 years up till now only two randomized clinical trials (RCT's) have been performed: one in patients with CRPS-1 and the other one in patients with Failed Back Surgery Syndrome FBSS (48) both of which provide limited (level 3) evidence that SCS relieves neuropathic pain (45). An RCT of SCS in patients with CRPS-1 (49) demonstrated that two thirds of the patients responded to this therapy with a $50 \%$ pain reduction after six months, as monitored by the Visual Analogue Scale (VAS). Unfortunately, still 1/3 of CRPS-1 patients receiving SCS treatment did not respond with a 50\% pain reduction for unknown reasons. The purpose of this review is to present an up to date overview of the basic physical and technical aspects of experimental and clinical SCS. This may be of use in our further understanding of this therapy and can give direction to future research and development. We need to find out what are the target neurons of SCS and how they can be electrically stimulated in order to give an optimal relief of neuropathic pain. In other words increased insights into the underlying mechanisms of SCS and at the same time further optimalization of the technical aspects of SCS may improve the success rate and effectivity of SCS.

\subsection{Proposed mechanisms of action in SCS}

In clinical spinal cord stimulation a longitudinal array of contacts (electrode) is placed into the dorsal epidural space either by a percutaneous technique or by means of a small laminectomy. Large myelinated primary afferent dorsal column fibres are depolarized and excited somewhere along their trajectory in the spinal cord at a Ranvier node (not at a peripheral receptor) near the electrode, leading to an action potential propagating in both directions (69):

1. Orthodromically in rostral direction to supraspinal centres: $A_{\beta}$-fibres directly projecting to the dorsal column nuclei and then further connected to the periaqaductal grey and the thalamus.

2. Antidromically via $A_{\beta}$-collaterals into the spinal cord target region where interneuronal connections exist with C-fibres and wide dynamic range (WDR) neurons in the dorsal horn.

3. Antidromically to the peripheral part of the $A_{\beta}$-dorsal root fibres. 
The activation of the dorsal column axons is thought to be responsible for the paresthesia experienced by patients during SCS. A supraspinal pathway of pain relief in SCS was also shown experimentally by El-Khoury et al (65). Here, dorsal column stimulation, rostral to selective dorsal spinal lesions at upper cervical levels resulted in significant pain relief (65). This shows that inhibitory effects of dorsal column stimulation on neuropathic pain can be attributed to the activation of brainstemmodulating centres via rostral projections of the dorsal column nuclei. However, it should be stressed that these findings cannot automatically be transferred to and form an explanation for the common approach in clinical as well as experimental studies where SCS is applied at lumbar spinal levels for treatment of neuropathic pain. Here experimental data strongly point to a spinal segmental mode of action. In a recent study on the localization of the electrodes on the dorsal column and the effect on pain relief in an experimental neuropathic pain model demonstrated that SCS of the dorsal columns at the level where the injured sciatic nerve fibres enter the spinal cord dorsal horn result in a much better pain relieving effect than SCS at more rostral levels. From this it was concluded that SCS in treatment of neuropathic pain acts almost exclusively through a segmental spinal site of action (70). In line with these findings are anatomical and biochemical observations in the lumbar dorsal horn: the antidromically propagated impulses are thought to induce changes at spinal levels as the balance of inhibitory and excitatory neurotransmitters in the dorsal horn is changed. Indeed, a segmental mode of action is supported by the fact that an increased neuronal activation in the spinal cord dorsal horn after SCS has been noted using c-Fos immuno-staining (71). In addition several experimental studies on the mechanisms of action of SCS showed an alteration of the chemical transmission in the spinal dorsal horn $(52,64,72,73)$ (Figure 1). There is evidence that the neuropathic pain syndrome, described as peripheral hypersensitivity with allodynia and hyperalgesia, is a result of central sensitization. Central sensitization is a result of neurochemical changes in the pain transmission in the dorsal horn mainly due to an increased release of the excitatory neurotransmitters glutamate and aspartate (74) and at the same time a loss of tonic GABA mediated inhibition.

Basically a decreased extracellular concentration of glutamate and the same time an increased extracellular GABA-concentration have been noted $(53,58)$ and this results in the suppression of hyperexcitable WDR neurons (55) (Figure 1). The WDR neurons are located in the dorsal horn laminae I, II, IV, V, VI and X and their main physiological function is to encode for the stimulus intensity of the received afferent input. WDR neurons play a key role as a modulator unit in the Gate Control theory for the relief of pain. In a recent paper (75) the effect of bipolar electrical SCS on the response properties of WDR neurons in the rat after L5 spinal nerve injury were examined. It was concluded that bipolar stimulation at the dorsal column but also after stimulation of the lumbar dorsal root attenuated the WDR hyperexcitability in nerve-injured rats and inhibited short-term neuronal sensitization (75). Other 
neurotransmitters, which might be related to either supraspinal or to spinal segmental mechanisms, that have been suggested to be involved in the pain relieving effect of SCS are serotonin, substance $\mathrm{P}$, adenosine and the muscarine receptor (M4 in particular) $(25,52,60,64,76)$. In view of the mechanism involved in the development of neuropathic pain (as reviewed by Berger et al (77) and/or in the mode of action of SCS recent developments from the experimental field cannot be neglected. The spinal dorsal horn has been reported to contain a 'silent' circuit between low-threshold afferent fibres and Nociceptive Specific (NS) projection neurons located in lamina I. Up to now, the composition of this circuit has been only partly described (78). Within this circuit excitatory interneurons in the innermost part of lamina II, which express the $\gamma$-isoform of protein kinase C (PKC- $\gamma$ ) are suggested to be important (Figure 1). Whereas these excitatory interneurons receive $A_{\beta}$-fibre innervations this implies that innocuous stimuli are thus able to activate PKC $-\gamma$ interneurons via $A_{\beta}$ fibres signalling (79). This information is not gated to NS projection neurons in the more superficial dorsal horn because PKC- $\gamma$ interneurons are under inhibition of glycinergic and gamma-amino-butyric acid (GABA)-ergic neuron (80). Activation of this silent circuit would result in the gating of innocuous stimuli to the NS projection neurons and thus, turn 'touch into pain'. Hence, 'touch can be turned into pain' by means of activation and/or sensitization of a silent dorsal horn circuit containing PKC- $\gamma$ interneurons, thereby gating $A_{\beta}$-fibre input to NS projection neurons.

Clearly future research should be focused at understanding when and how SCS interacts and modulates the pain circuit in the dorsal horn of the spinal cord (see Figure 1) 


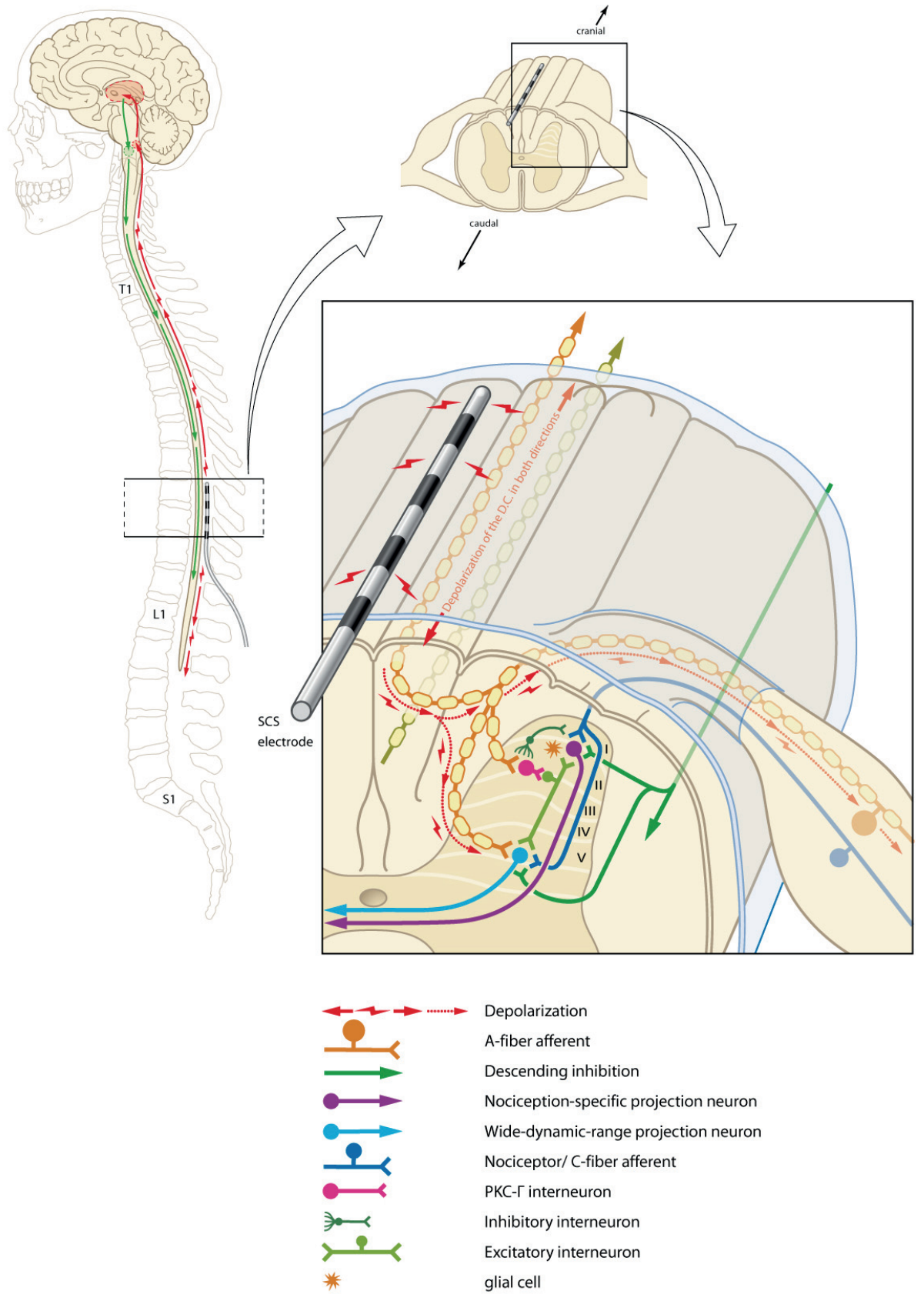


Figure 1: The spinal nociceptive network and mechanism of Spinal Cord Stimulation

The spinal nociceptive network and mechanism of Spinal Cord Stimulation. The spinal dorsal horn contains two major types of projection neurons: the NS located in the superficial laminae I and the WDR neurons located in the deeper dorsal laminae IV, V and VI. These projection neurons receive input from primary afferents, decending (aminergic) pathways, and spinal interneurons. Among the primary afferents are low-thresholds such as highly myelinated $A_{B}$ fibres originating from large-sized DRG neurons and further projecting into the dorsal columns to the dorsal column nuclei. Furthermore the projection neurons receive input from low treshold unmyelinated mechanoceptive C-fibres. The spinal nociceptive network also contains numerous interneurons, both of excitatory and inhibitory (GABA-ergic) nature, which modulate the processing of pain signals at the "gate" to the brain ("Gate-Control theory"). The spinal nociceptive network also contains a silent circuit between low threshold primary afferents and NS projection neurons. This circuit, which contains interneurons expressing PKC- $\gamma$ is normally inactive, but is thought to be activated under neuropathic conditions and, as such, turns 'touch into pain'. Electrical stimulation of the dorsal columns results in an action potential propagating in both directions: orthodromically in rostral directions to supraspinal centres and antidromically via $A_{\beta}$ collaterals into the spinal cord nociceptive network. The antidromic stimulation of the $A_{\beta}$ fibres has been shown to result in changed (decreased) release of glutamate of the primary (and presumably high-treshold C-afferents and at the same time an increased release of the inhibitory neurotransmitter GABA ('Gate Control Theory'). If and how the antidromic stimulation of large $A_{\beta}$ collaterals results in modulation and or sensitization of the silent circuit containing PKC- $\gamma$ interneurons is not yet known.

\subsection{Non-responders to SCS}

Non-responsiveness to SCS has been documented in CRPS type-1 patients as well as in an animal model for CRPS in about $1 / 3$ of the individuals. It should be noted that non-responsiveness in a clinical setting is based on a reduction of the pain as assessed with the VAS score whereas in an experimental settings this is almost exclusively measured with von Frey filaments an thus based on a reduction of the tactile hypersensitivity. Non-responsiveness to SCS may have several origins. A predictor for the absence of a significant response to SCS has recently been documented and shown to be related to the severity of allodynia experimentally (1) (Figure 2) as well as clinically (68). Non-responders to SCS in severe allodynic rats may be related to a severe type of central neuropathic derangement and may imply a disability to produce appropriate amounts of the inhibitory neurotransmitter GABA, either alone or accompanied by an increased loss of inhibitory interneurons (81). In this scenario modulation of dorsal horn neurons could have either little or no effect. In an experimental study non-responders to SCS could be turned into responders after an intrathecal application of low concentrations of the $\mathrm{GABA}_{\mathrm{B}}$ receptor agonist baclofen or other pharmacological agents (gabapentin, pregabalin, clonidine, adenosine) modulating GABA-ergic neurotransmission in the dorsal horn $(72,76,82-84)$.

Another explanation for non-responsiveness to mechanical (tactile) allodynia in the experimental setting or to pain in the clinical one may be of anatomical origin. Mechanical allodynia is reported to be primarily relayed by large myelinated fast $A \beta$ fibres which pass through the dorsal roots and gather in the ipsilateral dorsal col- 
umns and then terminate in the supraspinal nuclei (85) after which the signal proceeds to higher centres. Holsheimer et al demonstrated that during SCS only a small fraction (1\%) of the dorsal column (DC) fibres are depolarized (see section 5). It may be that if only a fraction of $A_{\beta}$ fibres that relay mechanical allodynia are depolarized, the pain signal can freely travel up the dorsal columns and the only possible inhibition of mechanical allodynia is a partial one in the dorsal column nuclei, resulting in also a partial pain relieving effect on mechanical allodynia.

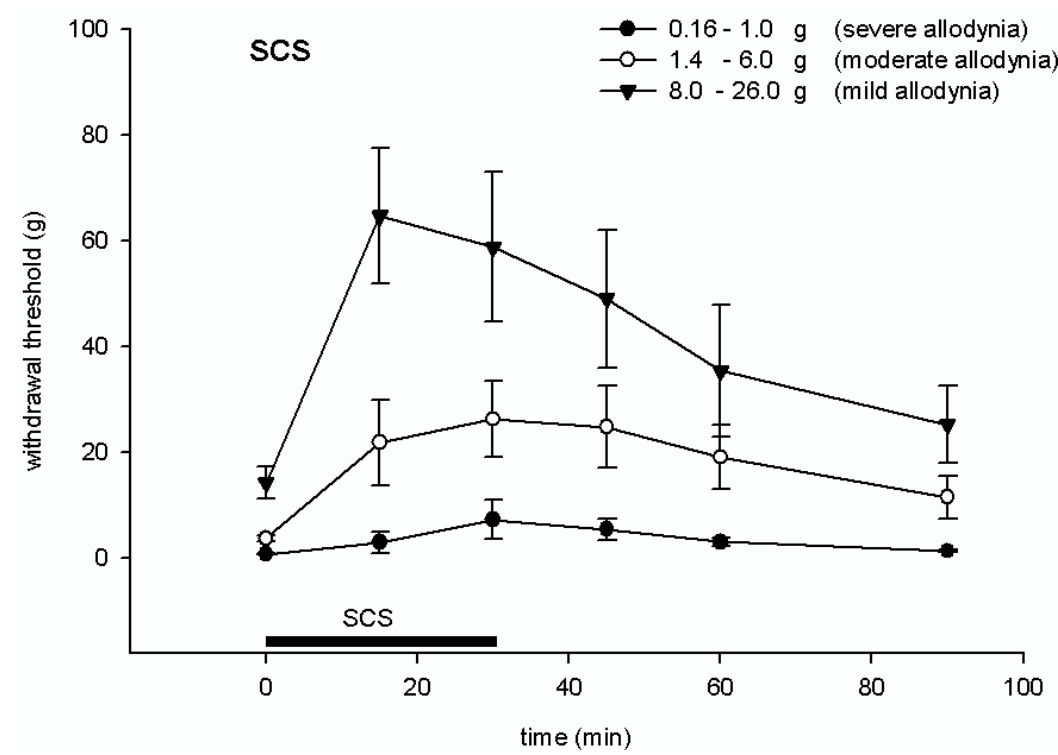

Figure 2: Effect of spinal cord stimulation in an animal model of neuropathic pain relates to degree of tactile "allodynia". SCS leads to a better and faster pain relief in mildly allodynic rats as compared with more severely allodynic rats. Reproduced from Smits et al., (2006) (1) Figure 3, page 544, with permission. Copyright Elsevier Ltd. (2006).

Furthermore, a lack of response to SCS may be related to a non-optimal application of SCS from a physical point of view. Firstly if the thickness of the dorsal CSF layer (dCSF) between the epidural electrode and the spinal cord is too large this will cause a predominant stimulation of dorsal root fibres leading to paresthesias in one or in two adjacent segments only (86). Other technical aspects of SCS as there are ; stimulation parameters, stimulation regime, electrode geometry, -configuration and localization are currently under investigation and may need to be optimized. In this respect it is important to consider and fine-tune technical parameters in the context of the mechanism of SCS. In other words: we need to explore exactly how SCS relieves pain in order to select the best electrophysiological stimuli for an optimal pain relief. Stimulation parameters currently used clinically and experimentally are only partly based on empirical studies whereas a thorough theoretical basis does often 
not exist. In this respect it is interesting to know that many basic neuroelectrophysiological principles of SCS were explored in a computer model by Holsheimer et al (87). These principles are often translatable to the clinic and have already led to improvements in the design of electrodes for clinical use.

In conclusion, the non responsiveness to SCS is still poorly understood. Differences in the stage or primary mechanism of the treated neuropathic pain could differ, possibly leading either to success or failure of SCS. Also differences of anatomy or physiology of the patient could lead to failure and it may well be that the response to SCS can still be improved with more effective stimulation parameters and hardware that can only be developed with a solid understanding of anatomy physiology and biochemistry of neuropathic pain and SCS.

\section{Anatomical and neurophysiological aspects of SCS}

\subsection{Functional anatomy of the dorsal columns}

The fasciculus gracilis and fasciculus cuneatus form a large bundle of axons located on the dorsal side of the spinal cord: the dorsal columns (DC). These axons carry information about fine touch, vibration and conscious proprioception from the body to the brainstem. The fasciculi consist of axons within a wide range of diameters including $A \beta$ fibres, all having their cell bodies in the ipsilateral dorsal root ganglia. Twenty-five percent of the $A \beta$ fibres run through the medial division of the posterior nerve roots and ascend as far as the medulla oblongata where they end in the cuneatus and gracilis nuclei (88). From the brainstem nerve fibres continue to the thalamus and cortex. This part of the pathway is often referred as the medial lemniscus pathway.

The fasciculus cuneatus consists of fibres from T6 up to $\mathrm{C} 1$ and is situated laterally in the DCs. The fasciculus gracilis containing fibres from spinal segment T7 down to S5 runs medially in the DCs. The DC fibres stay ipsilateral in the spinal cord and are distributed somatotopically. Fibres from higher spinal levels are positioned more lateral and lower spinal levels more medial in the DCs in a pallet formed fashion. Furthermore, it is known that the rat dorsal column is characterized by a very organized somatotopic arrangement: the primary afferents which enter the spinal cord and bundle into the dorsal column are initially located at the surface but rearrange to more ventro-medial areas at rostral spinal levels (89). This implies that in most neuropathic pain models which are based on sciatic nerve lesions $(56,57,90)$ the dorsal column afferents involved are located at the dorso-lateral surface of the dorsal column at vertebral level T13, whereas these afferents are located much deeper or ventro-medially into the dorsal column only a few spinal segments rostral. 
Apart from the primary afferent fibres the DCs also contain a major sensory pathway of thousands of second order post-synaptic projection neurons from the dorsal horn. These neurons originate from the nucleus proprius and from lamina III, IV, V and VI. The (SCS) stimulus evoked action potentials in the DCs are also propagated to the dorsal horn, where interneuronal modulation can take place. The dendrites of some of those neurons located into deeper dorsal layers extend even into lamina I and II and the axons often have local collaterals (91). The dorsal columns are separated from the SCS electrode by the dura mater and a layer of cerebrospinal fluid with a thickness varying between approximately 2.4 to $5.6 \mathrm{~mm}$ (92).

\subsection{Neurophysiology of SCS}

In SCS a relatively large electrode injects current into the extracellular space at a multi-cellular level. This ionic current passes neuronal membranes and causes either polarization or depolarization of the neuronal membrane. The myelinated nerve fibre consists of a cylindrical axon covered by an insulating myelin sheet which is interrupted by Ranvier nodes at regular intervals where ionic currents can pass the nerve fibre membrane. The myelinated axons have the electrical characteristics of a cable network including resistors and capacitators. This network can be used to calculate the influence of the stimulation induced electrical field on the nodal transmembrane voltages (93). The driving force of the change of the nodal transmembrane voltages leading to de- or hyperpolarization of the axon is called the activation function (AF). This AF is primarily determined by the second order difference of the nodal field potentials (94). A positive value of AF results in membrane depolarization whereas a negative value of AF results in membrane hyperpolarization. In SCS axons are depolarized by cathodic (electrode with negative charge) stimulation. When a nerve fibre approaches a cathode the AF value will rise and and the threshold stimulus needed for excitation will be reduced (95). The Ranvier node closest to the cathode will be excited first. The recruitment of nerve fibres by the SCS electrode is determined by the distance of the electrode from the nerve fibre as well as the nerve fibre diameter. In the conductive medium around the SCS electrode the current density is inversely proportional to the $2^{\text {nd }}-3^{\text {rd }}$ power of the distance, rapidly increasing the stimulation threshold as we move away from the electrode (96). The nerve fibre diameter has an effect on the value of AF of the nerve fibre as the potential differences between adjacent Ranvier nodes in large fibres are bigger, resulting in a greater peak value of AF, thus lowering the stimulation threshold. Hence, in SCS the first fibres to be recruited are large fibres located close to the electrode (at a few $\mathrm{mm}$ ). Increasing the stimulation amplitude leads to recruitment of larger fibres at some distance and smaller fibres close to the electrode. However, in SCS the amplitude is limited to approximately $40-70 \%$ of the paresthesia perception threshold, thus limiting recruitment of large axons further away from the electrode. In bipolar SCS DC- fibres with a diameter less than 9 micrometer are not be- 
ing recruited at all. With the largest DC-fibres having only 12 micrometers diameter, the total estimated amount of DC fibres recruited in SCS is only about $1 \%$ (97) of the total population.

In conclusion, SCS leads to bidirectional propagation of stimulus evoked APs of myelinated $A \beta$ fibres that are located in the highly organized structure of the dorsal column. This presumably leads to activation of the spinal dorsal horn pain network and consequently to pain relief. The electrical driving force of SCS rapidly declines as the distance of the SCS electrode to the nerve fibre increases and also depends on the size of the nerve fibre, causing a strong limitation in the actual number of DC fibres that can be depolarized.

\section{Technical aspects of SCS}

\section{1.a. Types of SCS stimulation electrodes}

SCS electrodes used in the clinic are either minimal invasive cylindrical catheter electrodes to be implanted percutaneously or surgical (paddle or plate) electrodes to be implanted by open surgery. Percutaneous catheter electrodes can be threaded many segments above the point of insertion and also allows displacement in a medial or lateral direction within the epidural space, allowing electrode placement near the target area of the dorsal columns (98). Percutaneous electrodes were originally used for a short stimulation trial only but over time they have evolved to devices that can also be anchored for permanent implantation. Today SCS electrodes whether percutaneous or laminectomy plate electrodes have multiple longitudinal contact arrays and can be programmed to connect the proper contacts as anodes and cathodes which allows adjustment of the focus of the electric field of stimulation.

\section{1.b. Anodic versus cathodic stimulation}

Cathodic stimulation is the most efficient way of stimulation as the cathodic (negatively charged) threshold for nerve fibre excitation is 3-7 times lower than the anodic threshold current (98). The much lower threshold for cathodic stimulation implies that the exact location of stimulation of the axons is determined by the position of the cathode.

\section{1.c. Preferred anode-cathode configurations}

In SCS the most common anode-cathode configurations are: monopolar (cathode), bipolar (cathode + anode) and tripolar stimulation $(+-+$, named guarded cathode or split anode). In monopolar stimulation only the cathode is close to the axons that are to be stimulated. The anode is located at a large distance and often the metal 
container of the stimulator is used for this purpose in such a way that the anodal field has no influence on the stimulation (98). Monopolar stimulation is characterized by a current injection from the cathode. If the anode is placed close to the cathode, thus creating a bipolar field, the largest current density is in the direction of the bipole axis. Consequently, the threshold current to stimulate nerve fibres parallel to the cathode-anode axis is selectively decreased. In a tripolar or guarded cathode configuration a central cathode is flanked by two anodes (Figure 3). If the two anodes and the cathode are close enough this will lead to superposition of the cathodic and anodic AFs and consequently the resultant increased positive cathodic $A F$ results in an increased depolarization and a decreased stimulation threshold.

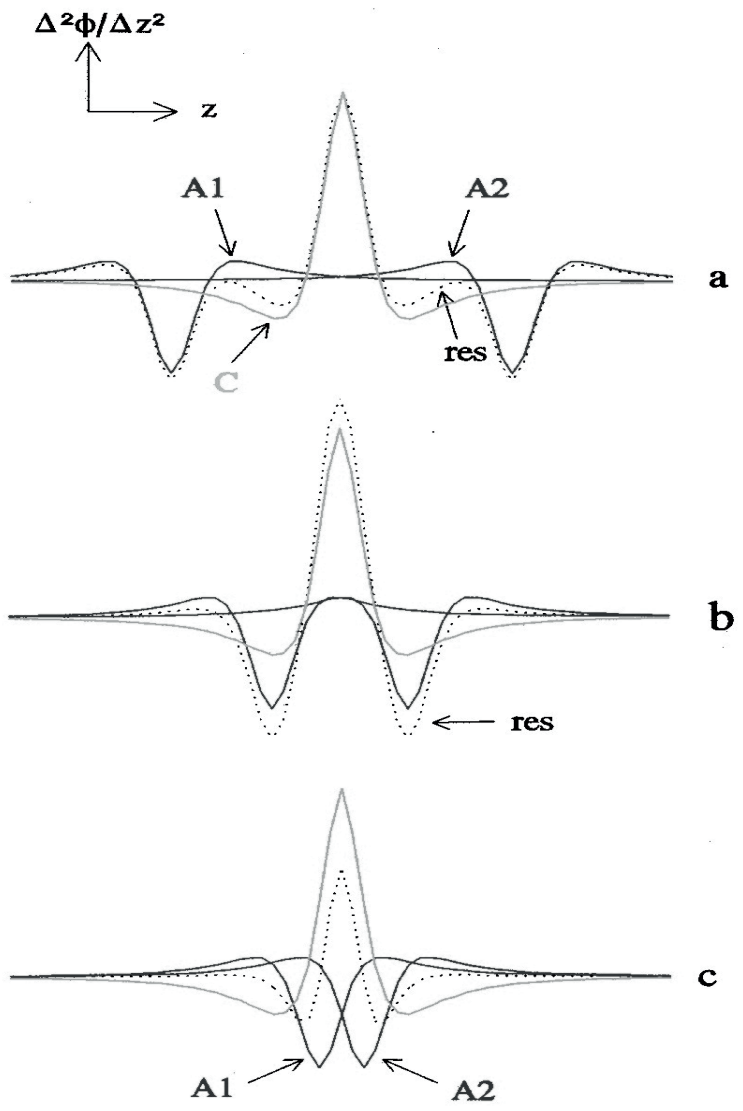

Figure 3: Superposition of the AFs corresponding to the cathode (C) and the two anodes (A1, A2) of a tripole ('guarded cathode'), dotted line (sum) is the superimposed AF; (a) large anode-cathode distance, cathodic peak value is not affected by the anodic AFs; (b) smaller electrode distance, summed cathodic peak value is larger (c) small electrode distance cathodic peak value is smaller. Reproduced with permission from Simpson BA (ed) Pain Research and Clinical Management, isbn 0-444-51258-6 Electrical Stimulation and the Relief of Pain, Volume 15, Figure 5, page 24. Copyright Elsevier Ltd (2003) (With permission) 


\section{1.d. Therapeutic range of stimulation}

For optimal therapeutic effect of SCS on neuropathic pain, the painful area has to be covered largely by the paresthesias elicited. In SCS the electrode is placed in the epidural fat on the dorsal side of the dura. The distance between the electrode and the spinal cord is similar to the dorsal CSF thickness (dCSF). This thickness varies among subjects. When dCSF is small the threshold current of dorsal root (DR) fibres exceeds the threshold current of dorsal column (DC) fibres. The perception threshold is low and the discomfort threshold is high, thus resulting in a high therapeutic range and a large paresthesia area. When dCSF is larger the perception threshold rises more steeply than the discomfort threshold (99), resulting in a reduced therapeutic range and a smaller paresthesia area. At mid and low thoracic spinal cord levels the relatively thick CSF layer results in an elevation in perception threshold while the discomfort threshold remains low. This leads to a decrease in the therapeutic range and increases patient side effects of discomfort or motor threshold at this level (100). This phenomenon also limits the management of widespread pain patterns as seen in CRPS-1, as only a limited portion of DC-fibres can be stimulated. Moreover if the distance between electrode and spinal cord is decreased the paresthesia coverage increases with a reduction in energy consumption. The mean value of the therapeutic range is about 1.4 .

The use of laminectomy electrodes instead of percutaneous electrodes in the low thoracic region of patients with Failed Back Surgery Syndrome leads to an improved pain relief; $90 \%$ versus $21 \%$ pain relief at 34 months respectively (101). The superiority of laminectomy electrodes can be explained by their larger mass causing a displacement of CSF, deceasing dCSF, lowering DC stimulation threshold and increasing therapeutic range and paresthesia coverage.

An additional advantage of laminectomy electrodes over percutaneous electrodes is their better fixation to the surrounding tissue thereby reducing the chances for electrode dislocation. Finally, less injected current is needed to activate DC fibres, because current is only injected on the anterior side of the plate electrode.

In addition to the effect of dCSF on paresthesia coverage other favourable conditions are: a bipole or guarded cathode, and a small centre distance between the contacts of the SCS leads ( $4 \mathrm{~mm}$ instead of 7-10 mm).

\section{1.e. Electrodes applied in animal experiments}

Experimental SCS studies are either fundamental neurophysiologic studies focusing on the neuronal pathways of SCS or clinically oriented translational studies on the mechanisms and the effects of SCS on neuropathic pain (see Table 1). Almost every translational experimental SCS study has been performed with a monopolar plate (platinum-iridium or solid silver) electrode with a rich variety in shapes and dimen- 
sions. In particular the hand-made silver electrodes used in the early experimental SCS studies were irregular in size and shape with a large thickness and often sharp edges. Thickness of the electrode may considerably effect the outcome: Meada et al using an electrode with a thickness of $0.35 \mathrm{~mm}$ and $2 \mathrm{~mm}$ wide reported spinal cord compression as a significant complication (102). Even when there is no sign of motor disturbance spinal cord compression is very likely to interfere in the sensory system and cause a bias in study results. It is far from unlikely that a considerable risk in dural damage exists using large or irregularly shaped electrodes causing CSF leakage and alteration of SCS electrophysiology thereby decreasing the accuracy of the SCS rat model. To decrease the probability of spinal cord compression, dorsal root/column stimulation or dural damage a $0.10 \mathrm{~mm}$ thin platinum-iridium rectangular plate electrodes with a width of $1 \mathrm{~mm}$ and very smooth edges was developed and tested in a rat experimental SCS model (1). Also the insulation of the wire connected to the electrode is crucial as even small damage to this wire can cause current leakage and ultimately result in non response. Recently, an even smaller version of this platinum-iridium electrode with a length and a width of 2.25 and 0.76 $\mathrm{mm}$, respectively, was introduced for SCS in a mouse model (103). In addition to the use of monopolar electrodes the first bipolar and quadrupolar electrodes have been developed for experimental work.

\subsection{Stimulation parameters}

Basically SCS results in the stimulation at a multi-cellular level because of the injection of current into the extra-cellular space around the neurons. Generally a nerve fibre generates an action potential if its membrane is depolarized by more than 15 $\mathrm{mV}$. A number of years ago all pulse generators for SCS were voltage-controlled devices that produce a potential difference between cathode (-) and anode (+). The resulting current depends on the applied voltage and the impedance between the two poles according to Ohms law: I (current) = V (voltage) / R (impedance). The relation between stimulation voltage and current is non-linear, which is caused by the complex impedance of the electrode-tissue interface (104). Therefore, a voltage pulse does not have a rectangular shape. During the last few years constant current devices have been introduced, which produce constant current between the cathode and the anode(s) which is not influenced by the impedance. The current injected creates a 3-dimensional electric field which can be represented graphically by isopotential and isocurrent density lines. The electrical stimulation applied to the target neurons is controlled by 3 parameters.

First, the amplitude (magnitude) of the pulse in Volt or Ampere. Secondly, the pulse width (the duration of the pulse in microseconds). Thirdly, the rate of the applied pulses in pulses per second (pps). Together the amplitude, the pulse width and the pulse rate determine the charge per second. The frequency used in most experi- 
mental and clinical studies is around 50-100 pps. Two clinical studies reported that the majority of patients preferred stimulation frequencies around $120 \mathrm{~Hz}$ while some preferred frequencies as high as $250 \mathrm{~Hz}(105,106)$. It has to be taken into account that after every action potential the sodium channels of a neuron are temporarily inactivated. This so-called refractory period is inversely related to the fibre diameter. Therefore, large diameter fibres will be able to follow higher stimulation rates, whereas the activation of smaller fibres will be desynchronized. Stimulation at very high pulse rates 300-500 pps may cause a depolarization block or neurotransmitter depletion. In this respect it is interesting to note that in an experimental SCS study on rats with neuropathic pain lower pulse rates (4- $60 \mathrm{pps}$ ) were reported to result into a better pain relief than using higher pulse rates (100- $250 \mathrm{pps)} \mathrm{(102).} \mathrm{In}$ experimental SCS a variety of stimulation parameters have been used (Table 1). So far in all experimental studies a pulse width of $0.2 \mathrm{~ms}$ was used. A constant current stimulator and stimulation amplitudes varying from $66 \%$ motor threshold (MT) (71 $\%$ of studies) to $90 \% \mathrm{MT}$ (in $24 \%$ of the studies) were used in nearly all studies, whereas only one study reported the use of an amplitude of $20-60 \% \mathrm{MT}$. From the few experimental SCS studies where motor thresholds were measured it seemed that the current amplitude necessary to elicit a motor response with an electrode implanted at T11 (implantation level verified by X-ray, (Figure 4) is larger as compared to T13. This may be due to differences in CSF space (dCSF). (see paragraph 3.1.d.)

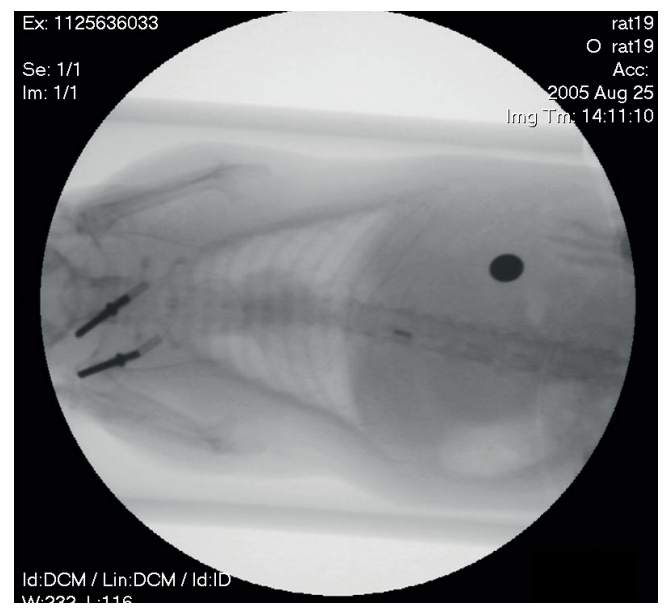

Figure 4: X-ray verification of electrode position at T13. Localization of the electrode was based on the position of the $13^{\text {th }}$ floating rib which is attached to the corpus of the $13^{\text {th }}$ vertebrae. Reproduced from Smits et al., (2006) (1) Figure 1, page 543, with permission. Copyright Elsevier (2006). 


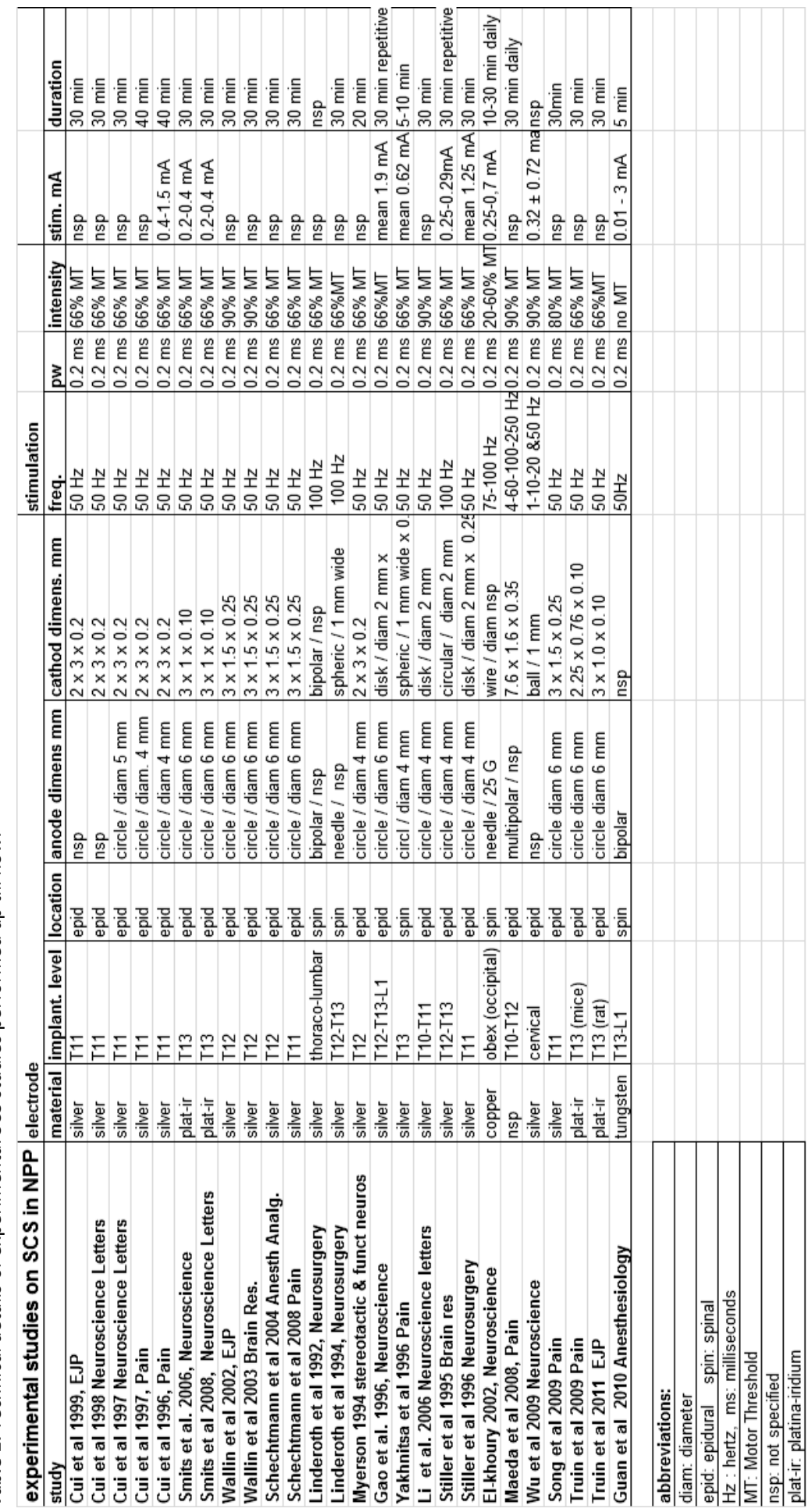




\subsection{Stimulation regimes: tonic stimulation and burst stimulation}

So far clinical SCS as well as SCS in experimental studies (see Table 1) was mainly performed by tonic stimulation. Tonic stimulation consists of electrical pulses, each one having the same pulse width, pulse rate and amplitude. Recent studies show that many central synapses are hardly or not at all signalling any single action potentials that arrives presynaptically. In fact most neurons require multiple synaptic input in order to respond and single action potentials are regarded by the central nervous system as noise. However, in central neurons depolarization by a short cluster (burst) of spikes results in an increase in presynaptic intracellular $\mathrm{Ca}^{2+}$ concentration. $\mathrm{Ca}^{2+}$ binding has not returned to baseline on arrival of the second stimulus and this so called facilitation will reliably lead to synaptic signaling (107). Bursts in the CNS are now regarded as intrinsic functional units of information and single bursts can produce Long Term Potentiation (LTP) or Long Term Depression (LTD). In a recent study tonic SCS (40 pps) was interspersed by short bursts of 5 spikes at 500 pps. This protocol was used in a small group of 12 patients and resulted in a paresthesia free pain suppression (108). These findings may substantially add to the efficacy of SCS in neuropathic pain, but the effectivity of this paresthesia free SCS remains to be proven in a controlled study.

\section{Other aspects}

\subsection{Development of neuropathic pain and timing of SCS}

Timing of SCS may considerably affect the outcome: SCS performed at an early stage of neuropathic pain may be clinically beneficial. Complete relief of CRPS-1 with SCS at 4 months after its onset was described in a recent case report (109). In an experiment on a rat model of neuropathic pain by Truin et al, early SCS 24 hours after Seltzer (56) partial sciatic nerve ligation resulted in an increased number of responders to SCS (positive response defined as greater than $50 \%$ pain relief) of $77 \%$ as compared to $38 \%$ responders in late SCS 16 days after nerve injury. Moreover in early SCS the duration of the effect of early SCS was also increased. On the other hand a study from our laboratory by van Eijs et al (paper submitted for publication) indicated that test stimulation in CRPS-1 patients at a mean disease duration of 7.5 months did not increase the response percentage to SCS: in $50 \%$ of patients test stimulation resulted in $50 \%$ pain relief which is comparable to earlier data of SCS in CRPS-1 patients (49). The experimental data on early timing of SCS in a rat model of neuropatic pain could not yet be confirmed by clinical studies. The time frame for development of neuropathic pain and successful early treatment with SCS in the rat model is days or hours. It is highly likely that, even if there is an increased response to early SCS in man, the stimulation probably has to be started much earlier than 
seven months after the onset of neuropathic pain as reported. A study by Schwartzman et al demonstrated that one year after onset of the disease the signs and symptoms of CRPS-1 are well developed and after this point progression is only moderate (110). The process of central sensitization in neuropathic pain may well have different phases that generate distinct therapeutic windows for different therapeutic options as related to their mechanism of action. Cui et el (76) demonstrated that an important mechanism in the relief of neuropathic pain by SCS is a partial resolvement of decreased GABA-ergic inhibition and increased excitation by glutamate (NMDA receptor) induced in the process of central sensitization. Eaton et al demonstrated that suppletion of GABA shortly after a $\mathrm{CCl}$ nerve injury significantly reversed mechanical hyperalgesia while late application failed to do so (111). In the experimental setting, timing of SCS earlier in the process of a still developing neuropathic pain possibly increases the chances of interfering into a more intact GABA-ergic system, leading to an increased number of responders. As central sensitization progresses more permanent changes in the GABA-ergic system may reduce the chance of SCS induced GABA-ergic inhibition.

Over the years clinical SCS has evolved more to an end stage therapy that is used in well selected patients with intractable neuropathic pain, primarily due to the invasive nature and the high costs of SCS. It is however far from unlikely that SCS earlier on in the process of neuropathic disease results into an increased number of responders.

\subsection{Repetitive Stimulation}

In general experimental studies are based on one single SCS treatment applied either early or late after the development of neuropathic pain In this context it is important to note that recent experimental studies have shown that repetition of the stimulation in itself results in a better pain reducing effect (102). In this study a repetitive 30 minutes per day SCS is applied for four day. A critical note concerning the latter publication is that the pain relieving effect of repetitive SCS in this paper is expressed as area under the curve which makes the absolute effect uncertain or at least less comparable to other experimental work.

\section{The development of a computer model for SCS}

Holsheimer and colleagues developed a computer model to simulate the SCSinduced electric field and the response of myelinated nerve fibres $(87,93)$. From this model it was calculated that the stimulus amplitude ratio for DC and Dorsal Root (DR) fibres is strongly influenced by the anode-cathode configuration (mono-, bi- or tripolar) and the geometry of the electrode (size and longitudinal separation of the contacts). The computer model represents both the geometry and the electrical 
conductivities of the constituting anatomical structures at 3 different spine levels. The intravertebral geometries were based on earlier human MRI studies. From these computer models it was calculated that the thickness of the dorsal CSF layer, varied between 2.4 and $5.6 \mathrm{~mm}$, and is is the main factor determining the perception threshold and paresthesia coverage in spinal cord stimulation (86). Increasing the CSF thickness raises the threshold and reduces the paresthesia coverage. Furthermore it was documented that a lateral asymmetry of less than $1 \mathrm{~mm}$ with respect to the spinal cord midline gives a significant reduction of perception threshold because the cathode is close to a right or left side DR and may result in unilateral (segmental) paresthesiae.

1. The computer model by Holsheimer and colleagues allows the design of an optimal electrode geometry, contact separation, contact size,) and configuration (mono-, bi-or multipolar) for SCS under various stimulation conditions with a longitudinal and/or transverse contact array, both surgical and percutaneous) (100). The development of a computer model has led to the following recommendations and clinical validation for human longitudinal contact array electrodes. Tripolar (guarded cathode or split anode) stimulation with one central cathode placed at the physiological midline provides the most efficient stimulation of the dorsal columns.

2. The contact centre separation is the most critical parameter and should be between 4 and $4.5 \mathrm{~mm}$.

3. Minimal electrode contact surface should be $6 \mathrm{~mm}^{2}$, according to FDA regulations regarding max. current density and max. charge per pulse (112),

4. The contact length should be between 1,5 and $3.0 \mathrm{~mm}$.

5. Using a laminectomy electrode the contact should be approximately $4 \mathrm{~mm}$ wide.

The calculated optimal electrode geometry from the model was later confirmed by clinical data $(113,114)$ Many currently available electrodes have larger contact surfaces (approximately $12 \mathrm{~mm}^{2}$ ) and contact centre separation $(7 \mathrm{~mm})$ (115). Electrodes with a reduced contact separation $(5 \mathrm{~mm})$ appeared to have a threefold increase in therapeutic range when compared to conventional electrodes contact separation $(9-10 \mathrm{~mm})(87)$. In patients a statistically significant preference for the guarded tripole electrode was reported (114).

\section{How to increase the success rate of SCS at the expense of non- response}

One aspect in the non response to SCS therapy may be the timing of SCS. New experimental data point out that SCS should possibly be performed earlier which then might interfere with the process of central sensitization of pain neurons in the spi- 
nal dorsal horn. Current SCS-treatment in CRPS and/or FBSS patients occurs at late stages in the disease when central sensitization is much more maturated, longlasting and even irreversible (see section 4.1).

Another aspect of non response in SCS is the presence of mechanical allodynia, which has some predictive value for the outcome of SCS (see section 1.3). In the future the identification of more possible signs, symptoms or markers that predict the outcome of SCS in advance is crucial as this may decrease non response to SCS by improved selection criteria. The increased non-response in the presence of mechanical allodynia could also be explained in terms of basic mechanisms and subscribes the need for more experimental work on this subject as understanding of the non-response to SCS might form the key in the development of innovations to improve the response to SCS.

From this review it is suggested that another major improvement in the non response to SCS in the treatment of neuropathic pain can almost certainly be expected from optimization of the technical aspects of SCS. The SCS electrode is the interface between the electrical signal of the stimulator and the neuronal tissue of the dorsal columns/roots, many electrode specific basic technical aspects have already been calculated from the computer model of Holsheimer and colleagues and were often validated clinically afterwards. For the success and reproducibility of experimental SCS certain basic recommendations can be made. As in the clinical setting the material, size, shape, thickness and uniformity of the experimental SCS electrode is crucial to the effectivity of SCS as well as the reproducibility of results and the comparability of the different studies. So far in experimental studies a variation of different electrodes (see Table 1) were used in the past, often irregular in shape and with a considerable variation in size. Other electrodes had a thickness of more than $0.3 \mathrm{~mm}$ resulting in spinal cord compression. Using thin $0.10 \mathrm{~mm}$ platinum-iridium electrodes we obtained highly reproducible results in a rat model of SCS (1). As to the stimulation parameters much research still lies ahead, in particular when we take into account that the traditional clinical tonic stimulation parameters ( $f=50 \mathrm{~Hz}$, pulse width $0.2 \mathrm{~ms}$ ) were copied to the experimental setting and remained unchanged for almost two decades (see Table 1). We can conclude that it is now time to start exploring the effects of other SCS parameters and SCS stimulation regimes that may enhance the understanding and effectivity of SCS (112).

From a basic scientific point of view it is important to detect the main target neuron(s) in SCS and how and which fibres are actually stimulated. From Figure 1 it can be deduced that besides the well known GABA-ergic (83) and Glutamatergic cells involved in the mechanism underlying SCS in treatment of neuropathic pain $(25,77)$, also other cells like the PKCy cells, the excitatory interneurons as well as various types of glial cells located in the dorsal horn of the spinal cord probably will play an important role in the modulation of the SCS evoked signal A broad range of tonic and burst stimulation regimes should be tested in the future. However, the 
concept of a SCS target pain neuron that needs to be stimulated in an optimal way may call for the approach of 'listening' more to the nervous system instead of offering 'noise' to it in order to find out physiological parameters of the electrical communication in neuronal cells and come up with a more goal directed approach to SCS parameters.

\section{Conclusion}

SCS undoubtedly is a valuable therapy for intractable neuropathic pain in CRPS-1 and

FBBS. However the fact that still $40 \%$ of the patients do not respond to SCS remains unexplained. Based on our review we conclude that the following steps, in a combined translational research effort, need to be taken:

1. Predictors: A search for predictors of a successful outcome of SCS. In this respect recent experimental and clinical data on the relation between the severity (or presence) of mechanical allodynia and success of SCS are hopeful.

2. Computer modelling: As SCS of CNS fibres is a very complex system future computer modelling will be needed in our effort to increase the effect or the number of responders in SCS. New findings on the basic neurophysiology, biochemistry and circuitry of SCS may be inserted into a computer model to calculate optimal SCS settings(116).

3. Experimental research: So far the SCS rodent models have proven to be clinically relevant and therefore more experimental research should be performed in order to elucidate the mechanisms behind response and non-response. From our limited understanding of the mechanisms involved important progress has already been made as it became clear that both experimentally and clinically the therapeutic effect of SCS can be potentiated by a simultaneous pharmacological intervention. 



\section{Chapter 3}

\section{Effect of Spinal Cord Stimulation in an Animal Model of Neuropathic Pain Relates to Degree of Tactile "Allodynia"}

Helwin Smits, Camilla Ultenius, Ronald Deumens, Guido C. Koopmans, Wiel M.M. Honig, Maarten van Kleef, Bengt Linderoth, Elbert A. J. Joosten 


\begin{abstract}
Spinal cord stimulation (SCS) is an established treatment for chronic neuropathic pain. However, in recent studies conflicting results regarding the effect of SCS were noted in a selected group of patients suffering from Complex Regional Pain Syndrome and mechanical allodynia. In the present study we investigated the pain relieving effect of SCS in a rat experimental model of neuropathic pain as related to the severity of mechanical allodynia. Adult male rats $(n=45)$ were submitted to a unilateral sciatic nerve ligation. The level of allodynia was tested using the withdrawal response to tactile stimuli with the von Frey test. A portion of these rats developed marked tactile hypersensitivity in the nerve-lesioned paw (von Frey test), similar to "tactile allodynia" observed after nerve injury in humans. Prior to SCS treatment the rats were subdivided into three groups based on the level of allodynia: mild, moderate and severe. All allodynic rats were treated with SCS $(n=27)$ for $30 \mathrm{~min}(\mathrm{f}=5 \mathrm{HHz}$; pulse width $0.2 \mathrm{~ms}$ and stimulation at $2 / 3$ of motor threshold) at 16 days post-injury. Our data demonstrate a differential effect of SCS related to the severity of the mechanical allodynia. SCS leads to a faster and better pain relief in mildly allodynic rats as compared to the more severely allodynic rats. Thus, we suggest that the selection and subdivision of patient groups similar to those defined in our experimental setting (mild, moderate and severe allodynic) may provide better pre-treatment prediction of possible therapeutic benefits of SCS.
\end{abstract}




\section{Introduction}

Spinal cord stimulation (SCS) as a treatment for neuropathic pain in patients was first described by Shealy et al in 1967 and has since become an established treatment for chronic intractable neuropathic pain (49,50,117-120). At present, more than 22.000 stimulation devices are implanted worldwide for this purpose each year. Interestingly, no large randomized clinical multi-centre trial (RCT) describing the effects of SCS on identified populations of patients with neuropathic pain has been published. Only for patients with CRPS (Complex Regional Pain Syndrome) it was demonstrated that SCS reduced pain and improved health-related quality of life $(49,50)$. CRPS patients are, in addition to spontaneous pain, not seldom affected by increased responses to noxious stimuli, allodynia to touch and hyperalgesia (121123). In a follow-up study by Kemler et al. (2001), no long-term effects of SCS on detection and pain thresholds of severe allodynic CRPS patients were noted. In contrast, allodynia relief was reported to be correlated with relief of the spontaneous pain in CRPS type I patients (123). Therefore, the clinically highly relevant question on the role of SCS in pain relief as related to the degree of allodynia still remains unsettled. Hence, as a first step we aimed to study the role of SCS in pain relief as related to the severity of mechanical allodynia in an experimental model of neuropathic pain. In the present study neuropathic pain was induced through the unilateral ligation of about half of the sciatic nerve at high thigh level (i.e.Seltzer model) (56). The unilateral ligation is known to result in touch-evoked allodynia and hyperalgesia as assessed using von Frey filaments (56). Then, neuropathic rats were treated with a miniature system for SCS $(51,124)$. Several experimental studies have been performed in this model focusing on the understanding of possible mechanisms underlying SCS $(53,55,58,76,84,125-128)$. The present data demonstrate a differential effect of SCS as related to the severity of the mechanical allodynia in a neuropathic model of pain.

\section{Experimental Procedures}

\section{Animals}

In this study a total of 45 male Sprague-Dawley rats (300-400 grams, 12 weeks) were used. The animals were individually housed from week 12, under standard conditions with a 12/12-hour light/dark cycle (lights off from 18.00 to $6.00 \mathrm{~h}$ ) and free access to food and water. The study was approved by the ethical committee for animal research of the University of Maastricht (DEC protocol no.2005/034). 


\section{Surgery and Anesthesia}

All operations were carried out under sterile conditions and performed under general anesthesia. Induction was performed in a plexiglass induction chamber using 5 $\%$ isoflurane. Anesthesia was maintained with an inventor $400{ }^{\circledR}$ injection system vaporizer (Zevenaar, Holland) with an open mask system using 2,5\% isoflurane in a $1: 1$ mixture of air and $100 \%$ oxygen at a flow of $250 \mathrm{ml} / \mathrm{min}$. Body temperature was maintained at 37,5 $\mathrm{C}+/-0.5 \mathrm{C}$ with an automatic heating pad (custom made Maastricht University technical service using an IR Carell ${ }^{\circledR}$ thermostate (USA)). Analgesic drugs were not administered to minimize the risk of interference with the development of allodynia (129).

\section{Unilateral Sciatic Nerve Ligation (Seltzer model)}

A partial nerve ligation was made in the left hindpaw according to a the Seltzermodel previously described by Seltzer et al (56). Briefly, under optical magnification $(25 x)$ the left sciatic nerve was carefully freed from surrounding connective tissue. At a site where the sciatic nerve is still monofascicular (130), just distal to the posterior biceps semitendinosis, but proximal to the little fat pad that lies a few millimetres distal to this site, the nerve was fixed in place by carefully pinching the dorsal epineurium with microforceps. An 8.0 silk suture with a reverse cutting and $3 / 8$ curve micro needle (Braun, Germany) was put through it and tied in such a way that $1 / 3$ to $1 / 2$ of the nerve diameter was ligated tightly at its dorsal side.

\section{Assesment of neuropathic pain}

The level of allodynia was determined by testing the withdrawal response to tactile stimuli with von Frey filaments of varying thickness.

Before the test the animal was kept in an elevated plexiglass cage with a wire mesh floor and allowed to adapt for 10 minutes. Subsequently, von Frey filaments were applied through the wire mesh floor to the mid plantar surface of the hind paws until the filament bent slightly. We used 14 Touch-Test von Frey filaments, number 5 to 16 (North Coast Medical, Inc.Ca. USA) with a regularly calibrated stiffness corresponding to $0.16,0.40,0.60,1.0,1.4,2.0,4.0,6.0,8.0,10,15,26,60$, and 100 grams. The filaments are presumed to roughly represent a logarithmic scale of applied force and a linear scale of perceived force (information provided by the manufacturer: North Coast Medical Inc Ca, USA). The filaments were applied to each hind paw in an order of increasing stiffness and the withdrawal threshold of each individual hind paw was defined as the force (in grams) of the filament that induced 3 out of 5 positive responses (brisk withdrawal). The non-lesioned side served as a control, displaying little if any effect of the nerve injury, subsequently showing no effect of the SCS. 
The withdrawal thresholds were tested at baseline (pre-nerve lesion) and at 2 Days Post-operative (2 DPO), 7 DPO and 14 DPO after a unilateral partial sciatic nerve ligation of the left hind paw. After testing at day 14 the SCS system was implanted and the animals were allowed to recover for two days before start of stimulation.

\section{Definition of allodynia}

We divided the rats which displayed increased withdrawal reactions in the lesioned paw - "allodynic animals", in three different groups based on cut-off points in a consecutive order of filament stiffness. Severe allodynia was defined as a positive response to the filaments with a stiffness corresponding to 0.16 to 1.0 (g) (4 filaments $0.16 ; 0.40,0.60$ and $1.0 \mathrm{~g}$ ). Moderate allodynia was defined as a positive response to the filaments with a stiffness corresponding to 1.4 to 6.0 (g) (4 filaments 1.4, 2.0, 4.0 and 6.0) and mild allodynia was defined as a positive response to the filaments with a stiffness corresponding to 8.0 to 26 (g) (4 filaments 8.0, 10, 15 and 26). Withdrawal threshold before nerve lesion or in the non-lesioned paw was mostly $>60-70 \mathrm{~g}$ with the present von Frey equipment.

\section{Implantation of the spinal cord stimulation system}

The SCS system we used has earlier been described in detail $(52,53,58,73,84,128)$. Our system is similar but contains some minor modifications. In short, we used a monopolar stimulation system (custom made by Medtronic ${ }^{\circledR}$ ) with a platinumiridium rectangular plate micro cathode (dimensions $3.00 \times 1.00 \times 0.10 \mathrm{~mm}$ ), which was placed in the dorsal epidural space. The anode consists of a circular platinairidium circular plate with a diameter of $6.00 \mathrm{~mm}$ and a thickness of $0.10 \mathrm{~mm}$ and was placed in a subcutaneous pocket on the animals back. Both electrodes were connected to micro plugs with an insulated multi-strand stainless steel wire.

Under general anesthesia a small laminectomy was made at T13 and the cathode was inserted in the epidural space at this level with the wire secured to the spinous process of T12 to prevent movement of the electrode. The anode was placed subcutaneously and both wires were tunnelled to the animal's neck where the plugs were attached to the skin. Electrode positions were verified by means of X-ray (Pulsera mobile C-arm, Philips medical systems BV Holland) to control for the accuracy of the implantation procedure.

Two days after the implantation the implanted electrodes were connected to a Grass S 88 stimulator (Astro-med Grass Warwick USA) fitted with a Grass SIU-5 stimulus insulator and a Grass constant current unit.

Motor thresholds were assessed by gradually increasing the current amplitudes at the following stimulator settings: Frequency $2 \mathrm{~Hz}$, pulsewidth 0.2 milliseconds. When the motor threshold (MT), was reached symmetrical lower trunk muscle twitches were observed and the current was measured in $\mathrm{mA}$. The SCS current was 
then set at $2 / 3$ of the MT, with a frequency of $50 \mathrm{~Hz}$. and pulsewidth was maintained at $0.2 \mathrm{~ms}$. These stimulation parameters correspond closely to the parameters that are used clinically. When the rats were subjected to SCS they showed a minor short (freezing) response, but soon resumed normal behaviour. Spinal cord stimulation was applied for 30 minutes and the withdrawal thresholds to touch of both hind paws were assessed at $t=0,15,30,45,60$ and 90 minutes.

\section{Inclusion criteria}

During the experiment various criteria for inclusion of animals were used. Animals with a pre-nerve lesion baseline of repeated von Frey withdrawal thresholds below 60 grams were not included. Furthermore, if the X-ray analysis showed an incorrect position of the electrode (i.e. not at T13) the animal was also not included in the study.

Finally, those animals without any elevation in von Frey withdrawal threshold above pre-stimulation threshold after SCS were defined as non-responders $(n=3)$ to the stimulation protocol and therefore excluded from the analysis.

\section{Statistics}

For each group we compared the withdrawal thresholds during and after SCS with the pre-stimulus withdrawal threshold ( $t=0$ minutes) using the dependent students$t$ test. Normally, $p<0.05$ is regarded as the level of significance. However, because multiple (5) comparisons were made, the level of significance was adjusted (posthoc correction) to $p<0.05 / 5=p<0.01$. In addition, we performed in each group a dependent students- $t$ test to see whether the maximal therapeutic effect in each group was significantly different from baseline in that same group. Here again, $p<$ 0.05 was regarded as the level of significance.

\section{Results}

\section{Total population characteristics}

From our 45 animals a total of 15 animals were excluded; 10 animals had pre-nerve lesion withdrawal thresholds below $60 \mathrm{~g}$, two animals were non-allodynic after the Seltzer procedure and three animals had an incorrect position of the electrode after X-ray verification (Fig.1). Hence a total of 30 "allodynic" animals received SCS. Before the Seltzer operation the withdrawal thresholds of these rats $(n=30)$ were 74.8 $\pm 3.6 \mathrm{~g}$ (mean $\pm \mathrm{SEM}$ ). Already at DPO 2 withdrawal thresholds showed a major significant decrease to $9.9 \pm 3.6 \mathrm{~g}(\mathrm{p}<0.0001)$ (mean $\pm \mathrm{SEM})$. At DPO 7 mean withdrawal thresholds were further reduced to $5.8 \pm 1.2 \mathrm{~g}$ (mean \pm SEM) and remained stable at this level up to SCS at day 16 (Fig 2A). 
In all a total of 30 allodynic animals received SCS. Three animals (10\%) did not show any increase in withdrawal thresholds above pre-stimulation levels and were thus regarded as non-responders to SCS.

In this population of SCS responders $(\mathbf{n}=\mathbf{2 7})$ mean pre-nerve lesion withdrawal threshold level was $74.8 \pm 3.8 \mathrm{~g}$. The mean pre-stimulation withdrawal threshold at DPO 16 was $5.7 \pm 1.3 \mathrm{~g}$. SCS resulted in a significantly increased mean withdrawal threshold of $26.4 \pm 6.6 \mathrm{~g}$ (mean $\pm \mathrm{SEM}$ ) ( $\mathrm{t}$-test compared to $\mathrm{t}=0, \mathrm{p}<0.003$ ) at $\mathrm{t}=15$ minutes. A further increase to $28.6 \pm 6.1 \mathrm{~g}$ (mean $\pm \mathrm{SEM}$ ) was observed at $\mathrm{t}=30 \mathrm{~min}$ (t-test compared to $t=0, p<0.0005)$. Thirty minutes after the initiation, SCS was terminated. The mean withdrawal thresholds decreased slowly to $24.3 \pm 5.2 \mathrm{~g}$ (mean \pm SEM) at 45 minutes ( $t$-test compared to $t=0, p<0.002$ ) and $16.6 \pm 4.3 \mathrm{~g}$ (mean \pm SEM) at 60 minutes ( $p<0.012$ ) (not-significant). (Fig 2B)

\section{SCS: Severe allodynia group}

In the severe allodynia group $(\mathbf{n}=7$ ) (defined as pre-stimulation withdrawal thresholds of $0.16-1.0 \mathrm{~g}$ ) the average pre-nerve lesion withdrawal threshold level was 82.8 $\pm 8.0 \mathrm{~g}$ (mean $+\mathrm{SEM})$. The mean pre-stimulation withdrawal threshold of the severely allodynic animals at DPO $16(\mathrm{t}=0)$ was $0.71 \pm 0.11 \mathrm{~g}$ (mean $\pm \mathrm{SEM})$. It should be noticed that in this group SCS did not lead to a statistically significant increase of withdrawal thresholds. A maximum of $7.2 \pm 3.6 \mathrm{~g}$ (mean \pm SEM) was observed at the end of SCS at $t=30$ minutes. (Fig 3)

\section{SCS: Moderate allodynia group}

In the moderate allodynia group $(\mathbf{n}=\mathbf{1 4}$ ) (defined by pre stimulation withdrawal thresholds of $1.4-6.0 \mathrm{~g}$ ) mean pre-nerve lesion withdrawal threshold level was $71.4 \pm 5.0 \mathrm{~g}$ (mean \pm SEM). The mean pre-stimulation withdrawal threshold at DPO 16 was $3.6 \pm 0.5 \mathrm{~g}$ (mean $\pm \mathrm{SEM}$ ). At $\mathrm{t}=15$ minutes after SCS was initiated the mean withdrawal threshold increased to $21.8 \pm 8.0 \mathrm{~g}$ (mean $\pm \mathrm{SEM}$ ) ( $\mathrm{t}$-test compared to $\mathrm{t}=$ $0 ; p<0.03)$. At $t=30$ minutes the mean withdrawal threshold further increased to a maximum of $26.2 \pm 7.1 \mathrm{~g}$ (mean $\pm \mathrm{SEM}$ ) ( $\mathrm{t}$-test compared to $\mathrm{t}=0 ; \mathrm{p}<0.004$ ). After 45 and 60 minutes, the withdrawal threshold were not statistically significant elevated compared to pre-stimulation; $24.7+7.7 . \mathrm{g}$ (mean \pm SEM) and $19.0 \pm 6.0 \mathrm{~g}$ (mean \pm SEM), respectively (Fig 3). 


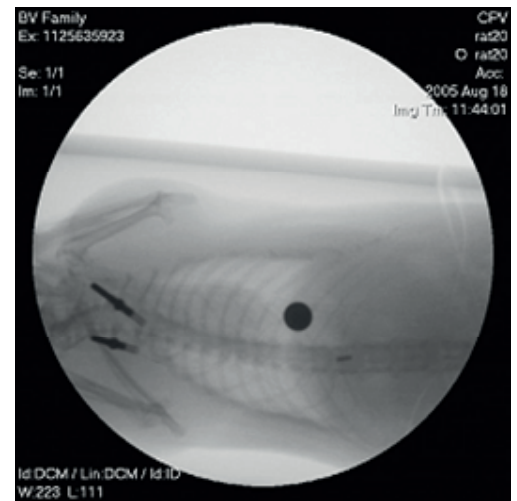

Fig 2A

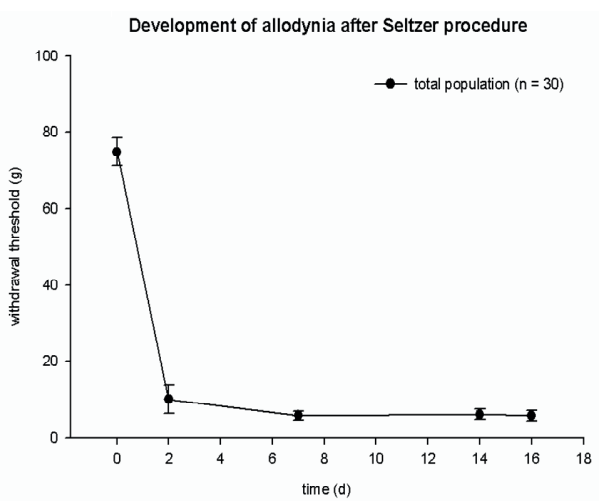

Fig 2B

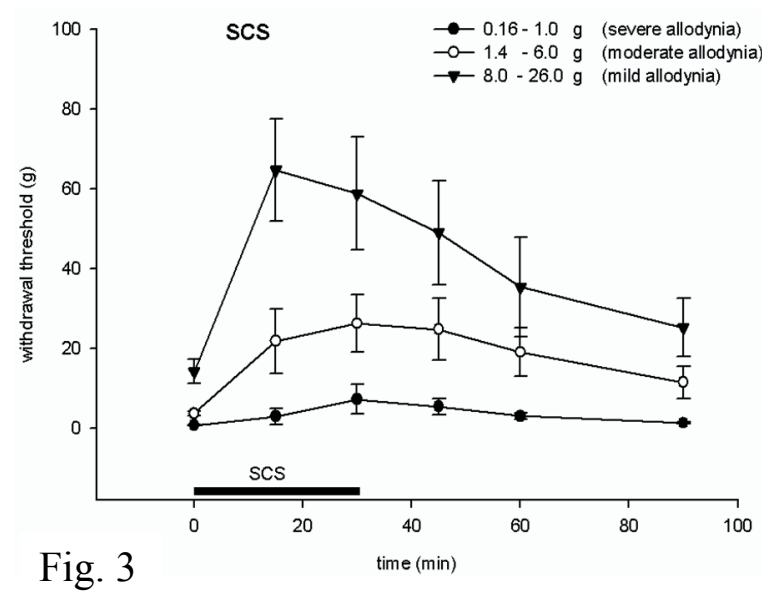

Figure 1: X-ray verification of electrode position at T13. Localization of the electrode was based on the position of the $13^{\text {th }}$ 'floating' rib which is attached to the corpus of the $13^{\text {th }}$ vertebrae.

Figure 2: Total group results. A. Changes in foot withdrawal threshold to von Frey hair stimulation after unilateral sciatic nerve ligation $(n=30)$. B. SCS affects the mean foot withdrawal threshold to von Frey hair stimulation in allodynic rats $(n=27)$. Mean pre-stimulation withdrawal threshold 16 days after unilateral sciatic nerve ligation and at start of the SCS $(t=0)$ were $5.7 \pm 1.3 \mathrm{~g}$; this threshold increased significantly $\left(^{*}\right.$ $\mathrm{p}<0.05$ ) during SCS being elevated post-SCS for more than an hour.

Figure 3: The change in foot withdrawal threshold response to von Frey stimulation after SCS differs in relation to severity of mechanical allodynia.

\section{SCS: Mild allodynia group}

In the mild allodynia group $(n=6)$ (defined by pre-stimulation withdrawal thresholds $8.0-26.0 \mathrm{~g}$ ) mean pre-nerve lesion baseline withdrawal threshold level was $73.3 \pm 7.8 \mathrm{~g}$ (mean \pm SEM). The mean pre-stimulation withdrawal threshold at DPO 16 was $14.2 \pm 2.9 \mathrm{~g}$ (mean \pm SEM). Already 15 minutes after initiating SCS a major 
and statistically significant increase in this threshold was observed, which even returned to pre-neuropathy levels; mean withdrawal threshold $64.6 \pm 12.8 \mathrm{~g}$ (mean $\pm \mathrm{SEM}$ ) (t-test compared to $\mathrm{t}=0, \mathrm{p}<0.0067)$. After this the mean withdrawal threshold declined to $58.8 \pm 14.1 \mathrm{~g}$

(mean \pm SEM) at $\mathrm{t}=30$ and $49.0 \pm 13.0 \mathrm{~g}$ (mean \pm SEM) at $\mathrm{t}=45$ and $35.2 \pm 12.5 \mathrm{~g}$ (mean \pm SEM) at $\mathrm{t}=60$ minutes, which was not statistically significant compared to $\mathrm{t}=0$. (Fig 3)

\section{Discussion}

In this study we have made two important observations with respect to the effect of SCS and neuropathic pain related behaviour in an experimental model where animals were subdivided according to their grades of (mechanical) allodynia. First, the response to SCS differs with severity of mechanical allodynia: in the mild allodynia group a maximal SCS-effect with complete return to baseline withdrawal thresholds was noted. Animals defined as moderately allodynic also responded to SCS but the withdrawal thresholds only increased to one-third of the baseline levels. Severely allodynic rats, however, did not respond to the SCS. Secondly, the response to SCS differed in time in the various groups of allodynic rats: an optimal response in the mild allodynic rats was noted after 15 minutes whereas the response in the moderately allodynic rats peaked at 30 minutes after the initiation of the SCS. In conclusion: SCS leads to a faster and better pain relief in mildly allodynic rats as compared to the more severely allodynic rats.

In the context of SCS studies in experimental pain models the issue of responders versus non-responders is often raised $(73,125)$ but in a recent study variants of the Spared Nerve Injury (SNI) model (131) were examined and in these studies outcomes of SCS indicated a relation to the extent of axonal damage (132). As can be deduced from the present studies we also faced substantial numbers of nonresponding rats. It should be emphasized that in this setting a response can be regarded in two ways: 1 . the response to the unilateral sciatic nerve ligation, and 2 . the response to SCS.

\section{Responders to unilateral sciatic nerve ligation}

From the initial 45 animals that underwent ligation of the sciatic nerve, 10 were excluded because of an initial low baseline withdrawal threshold. From the remaining 35 animals, 26 (74\%) responded appropriately with a withdrawal threshold $<7$ (g) at DPO 16. In previous experimental studies using the same unilateral sciatic nerve ligation according to Seltzer (1990) the percentage of animals with a marked decrease in withdrawal thresholds $(\leq 7.5 \mathrm{~g})$ was often reported substantially lower, often down to $20 \%(53,73)$. However, in the initial study by Seltzer and co-workers, 
a higher frequency of low-range withdrawal thresholds (0-2 (g) $74 \%$ at DPO 27) was reported. Although it was suggested that phytoestrogens in a soy rich diet reduces tactile allodynia and mechanical hypersensitivity and thereby may affect the percentages of responders versus non-responders after nerve injury $(133,134)$, the exact localization of the ligation may as well be of pivotal importance. The sciatic nerve ligation should be placed at a height where the sciatic nerve is still monofascicular, between the position where the posterior biceps semitendinosis nerve branches off the common sciatic nerve and the little fat pad that lies a few millimetres distal next to the sciatic nerve $(56,130)$. Then ligation according to the procedure described by Seltzer and co-workers may result in an increased number of responders as demonstrated by the present experiments. Another factor of importance is the testing procedure and the equipment use, conditions that differ considerably between studies (131); see discussion in (132).

\section{Responders to SCS}

The majority of the previous animal experimental studies on SCS $(50,52,53,58,73,82$, $84,125,128$ ) focused primarily on the mechanisms of SCS in the relief of neuropathic pain. Basically responders to SCS were classified as animals in which SCS induced an increase in withdrawal threshold of $20 \mathrm{~g}$ or more and the reported fraction of responders using this criterium was usually approximately $50 \%(53,73)$. If we analyse our total population of animals that received SCS based on the same criterium as previously used in literature $(53,73)$ we observe that 11 out of $21(52 \%)$ animals showed an increase of $20 \mathrm{~g}$ with an initial withdrawal threshold $<8 \mathrm{~g}$. Also in line with the literature is the fact that in our total SCS rat population the maximal pain relieving effect was noted at $\mathbf{3 0}$ minutes after initiation of the stimulation followed by a relatively slow decrease in the effect (Fig.2B). This complies with previously reported effects of SCS in experimental pain models $(53,73)$. Finally in our experiment only 2 severe allodynic and one moderate allodynic rat (and thus a total of 3 out of 30) animals did not respond to SCS at all and for that reason were excluded from our study. It should be stressed that these exclusions did not affect our results in any form. The exclusion of non-responders to SCS is valid because also in the clinical situation patients who do not respond to a test stimulation will be excluded from SCS treatment.

Although SCS has been shown to be pain relieving in the experimental setting after a Seltzer unilateral sciatic nerve ligation, no discrimination has been made so far between the effects of SCS in relation to the severity of the pain-related behaviour. The most important finding of the present study is that our results demonstrate a differential effect of SCS within a total rat population which is subdivided into three groups: mildly, moderate and severely allodynic rats. This differential effect may have important clinical implications. 


\section{Clinical implications}

Pain relief in SCS as a therapy for neuropathic pain has previously been described experimentally and clinically $(49,53,58,59,123-125,135)$. As in a clinical setting, where a test stimulation is part of the standard procedure, we excluded the animals which did not respond to SCS ( $n=3)$. Also in line with the clinical situation we verified the position of the electrode by use of X-ray (Fig.1). Incorrect positioning of the electrode led to exclusion of three animals. It should be emphasized that routine verification and thus better localization of the electrode can result in a more optimal response to the SCS-treatment. In previous studies X-ray control has been performed only occasionally and only a physiological reaction to test stimulation has been the control $(53,58,73,128)$.

In our experiments we observed significant differences in relief of allodynia after SCS. In the healthy animals before sciatic nerve ligation, baseline levels did not differ significantly between our three defined groups of allodynia. The Seltzer sciatic nerve lesion results in a broad range of withdrawal thresholds, reflecting different levels of "allodynia" as described above. Also in the clinic a large variation in the grades of allodynia in patients with neuropathic pain is observed. In the light of the present experimental results we feel that the effect of SCS on different levels of allodynia should also be investigated clinically. This may help us in identifying patients that would benefit the most from SCS. In a clinical study by Kemler et al (2001) the effects of SCS on sensory characteristics in 24 patients with Complex Regional Pain Syndrome type I (CRPS type I) was investigated. From the results it was concluded that SCS had no long-term effect on detection and pain thresholds for pressure, warmth or cold and only a minimal effect on mechanical hyperalgesia. The patients referred to in this study were all characterized by a visual analogue score (VAS) > 5 and thus displayed at least "severe spontaneous pain" (136). Sensory characteristics like detection and pain threshold for pressure, warmth and cold were recorded at different time points up to 12 months. These clinical results may to some extent (regarding rat life-span) correspond to those observed in our severe allodynic rats-group: a group, which did not respond to the SCS. The observation by Kemler et al (2001) is in contrast to the outcome in a more recent study (123) where a marked SCS effect on the tactile allodynia was discovered. Thus, conflicting clinical observations exist.

In conclusion: our experimental data indicate the need for a study of SCS in patients subdivided into categories according to their level of allodynia. 

Chapter 4

Spinal Cord Stimulation of dorsal columns in a rat model of neuropathic pain: evidence for a segmental spinal mechanism of pain relief 


\begin{abstract}
Although Spinal Cord Stimulation (SCS) of the dorsal columns is an established method for chronic neuropathic pain, patients still suffer from a substantial level of pain. From a clinical perspective it is known that the location of SCS is of pivotal importance thereby suggesting a segmental spinal mode of action. However, experimental studies suggest that SCS acts also through the modulation of supraspinal mechanisms, which might suggest that the location is unimportant. Here we investigated the effect of the rostro-caudal location of SCS-stimulation and the effectiveness of pain relief in a rat model of chronic neuropathic pain. Adult male rats $(n=45)$ were submitted to a partial ligation of the sciatic nerve. The majority of animals developed tactile hypersensitivity in the nerve lesioned paw. All allodynic rats were submitted to SCS $(n=33)$ for $30 \mathrm{~min}(f=50 \mathrm{~Hz}$; pulse width $0.2 \mathrm{~ms})$. In one group $(n=16)$ the electrodes were located at the level where the injured sciatic nerve afferents enter the spinal cord (T13) and in a second group ( $n=17)$ the electrodes were positioned at more rostral levels (T11) as verified by X-ray. A repositioningexperiment of electrodes from T12 to T13 was performed in two animals. Our data demonstrate that SCS of the dorsal columns at the level where the injured fibres enter the spinal cord dorsal horn result in a much better pain relieving effect than SCS at more rostral levels. From this we conclude that SCS in treatment of neuropathic pain acts through a segmental spinal site of action.
\end{abstract}




\section{Introduction}

Spinal Cord Stimulation (SCS) for the treatment of chronic intractable neuropathic pain was introduced in 1967 (137). Today SCS of the dorsal columns is clinically used as a valuable treatment for neuropathic pain, as for instance in Complex Regional Pain Syndrome type-1 (CRPS-1) (49). Although the observed pain relief was significant the patients were not free from pain and still suffered with a substantial level of pain $(49,68,138)$.

From experimental neuropathic pain models it is suggested that SCS of the large $A B$ fibres in the dorsal columns acts both through the modulation of a spinal as well as a supraspinal mechanisms. A segmental spinal mechanism of pain relief was based on biochemical and histological analysis of the rat dorsal horn in SCS treated chronic neuropathic rats $(58,67,71,72,139)$. Evidence for a role of supraspinal mechanisms was presented by El Khoury and collegues (65) who demonstrated that SCS of the dorsal column nuclei after a dorsal column transection below the nuclei still induces relief of neuropathic pain. The latter findings suggest that the exact spinal level of SCS is unimportant as electrical stimulation of the dorsal column fibres would result in depolarization both orthodromically (supraspinal stimulation) and antidromically (spinal or segmental stimulation). However, the combination of significant differences in the pain relieving effect of SCS noted in experimental studies and a number of electrophysiological and anatomical principles makes the vertebral level of implantation of the electrode in relation to the pain relieving effect of SCS an important issue. The use of a computer model based on electrophysiological measurements combined with morphometric analysis of the dorsal columns lead to the conclusion that only a very small and superficially located fraction of the $A \beta$ fibres located in the dorsal column is actually stimulated and depolarized $(96,97)$. Furthermore, it is known that the rat dorsal column is characterized by a very organized somatotopic arrangement: the primary afferents which enter the spinal cord and bundle into the dorsal column are initially located at the surface but rearrange to more ventro-medial areas at rostral spinal levels (89). This implies that in most neuropathic pain models which are based on sciatic nerve lesions $(56,57,90)$, the dorsal column afferents involved are located at the dorso-lateral surface of the dorsal column at vertebral level T13, whereas these afferents are located much deeper or ventro-medially into the dorsal column only a few spinal segments rostral.

The present study was undertaken and based on the hypothesis that SCS acts through a segmental spinal mechanism of pain relief where SCS of the dorsal column at vertebral bony level T13 (or spinal cord segments L2-L5) will result in a more effective pain relief as compared to SCS treatment at T11. 


\section{Methods}

\section{Animals}

In this study a total of 42 male Sprague-Dawley rats (300-400 g, 12 weeks) were used. The animals were individually housed from week 12, under standard conditions with a 12-h light/dark cycle (lights off from 18:00-06:00 h) and free access to food and water. All experimental procedures were performed according to the recommendations of the European Commission and protocols were approved by the Committee on Animal Research of the Maastricht University (DEC \# 2008-144). Every attempt was made to minimize the number of animals and their suffering.

\section{Surgery and anesthesia}

All operations were carried out under sterile conditions and performed under general anesthesia. Induction was performed in a Plexiglas induction tube using $5 \%$ isoflurane. For maintenance of anesthesia we used an Inventor $400^{\circledR}$ injection system vaporizer (Zevenaar, Holland) with an open mask system (2.5\% isoflurane in a 1:1 mixture of air and $100 \%$ oxygen at a flow of $250 \mathrm{ml} / \mathrm{min}$ ). Body temperature was maintained at $37.5{ }^{\circ} \mathrm{C}+0.5{ }^{\circ} \mathrm{C}$ with an automatic heating pad (custom made Maastricht University technical service using an IR Carell ${ }^{\circledR}$ thermostat (Carell, Manheim, PA, USA)). No analgesic drugs were administered to minimize the risk of interference with the development of allodynia (129).

\section{Unilateral sciatic nerve ligation (Seltzer model)}

A partial sciatic nerve ligation was made in the left hind paw (90). Briefly, under optical magnification $(25 \mathrm{x}$ ) the left sciatic nerve was carefully freed from surrounding connective tissue. At a site where the sciatic nerve is still monofascicular, just distal to the posterior biceps semitendinosus, but proximal to the little fat pad that lies a few millimeters distal to this site, the nerve was fixed in place by carefully pinching the dorsal epineurium with microforceps. An 8.0 silk suture with a reverse cutting and $3 / 8$ curve micro needle (Braun, Germany) was put through it and tied in such a way that $1 / 3-1 / 2$ of the nerve diameter was ligated tightly at its dorsal side.

\section{Assessment of neuropathic pain}

The degree of allodynia was assessed by testing the withdrawal response to tactile stimuli with von Frey filaments of varying thickness. Before testing the animal was allowed to adapt for $10 \mathrm{~min}$ in the elevated Plexiglas von Frey testing cage with a wire mesh floor. Subsequently, von Frey filaments were applied through the wire mesh floor to the midplantar surface of the hind paws until the filament bent slightly. We used 14 touch-test von Frey filaments, numbers 5-16 (North Coast 
Medical, Inc., Morgan Hill, CA, USA) with a regularly calibrated stiffness corresponding to $0.16,0.40,0.60,1.0,1.4,2.0,4.0,6.0,8.0,10,15,26,60$, and 100 g. The filaments are presumed to roughly represent a logarithmic scale of applied force and a linear scale of perceived force (information provided by the manufacturer: North Coast Medical Inc.). The filaments were applied to each hind paw in an order of increasing stiffness and the withdrawal threshold of each individual hind paw was defined as the force (in grams) of the filament that induced three of five positive responses (brisk withdrawal). The non-lesioned side served as a control, displaying little if any effect of the nerve injury, subsequently showing no effect of the SCS. The withdrawal thresholds were tested at baseline (pre-nerve lesion) and at 2 days post-operative (2 DPO), 7 DPO and 14 DPO after the unilateral partial sciatic nerve ligation of the left hind paw. After testing at day 14 the SCS system was implanted and the animals were allowed to recover for two days before start of stimulation.

\section{Definition of allodynia}

As in our previous work $(1,71)$ the rats which displayed decreasing withdrawal threshold in the lesioned paw or allodynic animals, were divided into three different groups based on cutoff points in a consecutive order of filament stiffness. Severe allodynia was defined as a positive response to the filaments with a stiffness corresponding to $0.16-1.0$ (g) (four filaments: $0.16,0.40,0.60,1.0 \mathrm{~g}$ ). Moderate allodynia was defined as a positive response to the filaments with a stiffness corresponding to 1.4-6.0 (g) (four filaments 1.4, 2.0, 4.0 and $6.0 \mathrm{~g}$ ) and mild allodynia was defined as a positive response to the filaments with a stiffness corresponding to 8.0-26 (g) (four filaments 8.0, 10, 15 and $26 \mathrm{~g}$ ).Withdrawal threshold before nerve lesion or in the non-lesioned paw was mostly $>60-70 \mathrm{~g}$ with the present von Frey equipment.

\section{Randomization of study groups T11 or T13 and blinding of sensory testing}

At the beginning of the study each animal was randomly assigned for implantation of the SCS electrode either at the T11 or the T13 level. After group assignment the animals received a clear tail number and were randomly mixed to prevent any group differences in housing. All von Frey tests and spinal cord stimulations were carried out by an experienced investigator (HS) who was blinded for the previous sensory state and the actual study group of the animal.

\section{Implantation of the SCS system}

The SCS system we used has earlier been described in detail $(1,71)$ (custom made by Medtronic $\left.{ }^{\circledR}\right)$ with a standardized platinum-iridium rectangular plate microcathode $(3.00 \times 1.00 \times 0.10 \mathrm{~mm})$, which was placed in the dorsal epidural space. The dimensions of the cathode are based on MRI images of the rat spinal cord and were chosen specifically to exactly fit above the dorsal column area in the rat. The thickness 
of the cathode is small enough not to cause compression of the spinal cord and is probably helpful in stabilizing the medial lateral position of the electrode as the CSF under the dura produces an even pressure pushing the electrode to the arched vertebral bone above it. The anode consists of a circular platinum-iridium circular plate (diameter of $6.00 \mathrm{~mm}$, thickness of $0.10 \mathrm{~mm}$ ) was placed in a subcutaneous (s.c.) pocket on the animals back. Both electrodes were connected to microplugs with an insulated multi-strand stainless steel wire. Under general anesthesia and with the help of X-ray visualisation a small laminectomy was made at T13 or T11 depending on the group the animal was assigned to. The cathode was inserted in the epidural space exactly in the midline of the spinal cord at this level with the wire firmly secured with histo-acryl tissue glue into a prepared groove in the adjacent spinous process to prevent any rostral caudal or medial lateral movement or dislocation of the lead. The anode was placed subcutaneously and both wires were tunnelled to the animal's neck and the plugs were secured to the skin. Electrode positions were verified by means of X-ray (Pulsera mobile C-arm, Philips Medical Systems BV, Holland) to control for the accuracy of the implantation procedure. Specifically; the T11 and T13 vertebrae were identified by counting the ribs of the animal using X-ray. The 11-th rib is attached rostral at the corpus of the 11-th thoracic vertebrae and the 13-th rib to T13 in the same way. Two days after the implantation the electrodes were connected to a Grass S 88 stimulator (Astro-med, Grass, Warwick, RI, USA) fitted with a Grass SIU-5 stimulus insulator and a Grass constant current unit. Motor thresholds (MT) were assessed by gradually increasing the current amplitudes at the following stimulator settings: frequency $2 \mathrm{~Hz}$, pulse width $0.2 \mathrm{~ms}$. When the MT, was reached symmetrical lower trunk muscle twitches were observed and the current was measured in $\mathrm{mA}$. The SCS current was then set at 2/3 of the $\mathrm{MT}$, with a frequency of $50 \mathrm{~Hz}$. and pulse width was maintained at $0.2 \mathrm{~ms}$. These stimulation parameters closely correspond to the SCS parameters used clinically. When the rats were subjected to SCS they showed a minor short (freezing) response, but soon resumed normal behaviour. SCS was applied for $30 \mathrm{~min}$ and the withdrawal thresholds to touch of both hind paws were assessed at $t=0,15,30,45$, 60 and $90 \mathrm{~min}$. After SCS and von Frey assessment the animals were euthanized with pentobarbital $150 \mathrm{mg} / \mathrm{kg}$ (1:10 solution) intraperitoneal (i.p.). Then a second Xray was performed to exclude possible dislocation and thus verify the correct position of the electrode during the actual SCS.

For the electrode replacement experiment $(n=3)$ the electrode was initially placed at T12 as verified by X-Ray. One day after the stimulation the rats were reoperated and the electrode re-positioned at T13 (verified by X-ray) followed by stimulation one day later. 


\section{Inclusion criteria}

During the experiment various criteria for inclusion of animals were used. Animals with a pre-nerve lesion baseline of repeated von Frey withdrawal thresholds below $60 \mathrm{~g}$ were not included $(\mathrm{n}=2)$. Animals displaying withdrawal thresholds greater than $26.0 \mathrm{~g}$ at DPO 14 after sciatic nerve injury were not included in the study ( $\mathrm{n}=$ 5). Furthermore, those animals without any increase in von Frey withdrawal threshold above pre-stimulation threshold after SCS were defined as non-responders ( $\mathrm{n}=$ 3 ) to the stimulation protocol and therefore excluded from the analysis. The T11 group contained two non-responders and the T13 group contained one nonresponder. Finally, if the X-ray analysis (see section: implantation) resulted in an incorrect position of the electrode (i.e. not at the intended T13 or T 11 level) the animal was excluded from the study $(n=0)$.

\section{Statistics}

The tactile withdrawal thresholds in grams to the von Frey values were converted to the percentage of Pre-Injury Paw Withdrawal Thresholds (\% of PIPWTH) and are presented as mean \pm standard error of the mean (SEM). Statistical analysis were carried out using SPSS version 13 software. Data were tested for normality and student's paired t-test. Normally, $\mathrm{p}<0.05$ is regarded as the level of significance. However, because multiple comparisons were made (five), the level of significance was adjusted (post-hoc correction) to $p<0.05 / 5=p<0.01$. Statistical analysis of the area under the curve (AUC) was performed using an unpaired t-test or one-way ANOVA and then $p<0.05$ was considered to be statistically significant.

\section{Results}

\section{Total population characteristics}

From the 45 animals a total of 10 animals were excluded; two animals had prenerve lesion von Frey withdrawal thresholds below $60 \mathrm{~g}$, five animals were nonallodynic after the Seltzer procedure and three animals (two animals from the T11 group and one animal from the T13 group) were non-responders to SCS as they did not respond to SCS with any increase in withdrawal thresholds above prestimulation levels. Hence a total of 33 animals (T13: $n=16 ; T 11: n=17$ ) were included into the analysis. In all animals the electrode positions were verified by X-ray (Fig.1) 


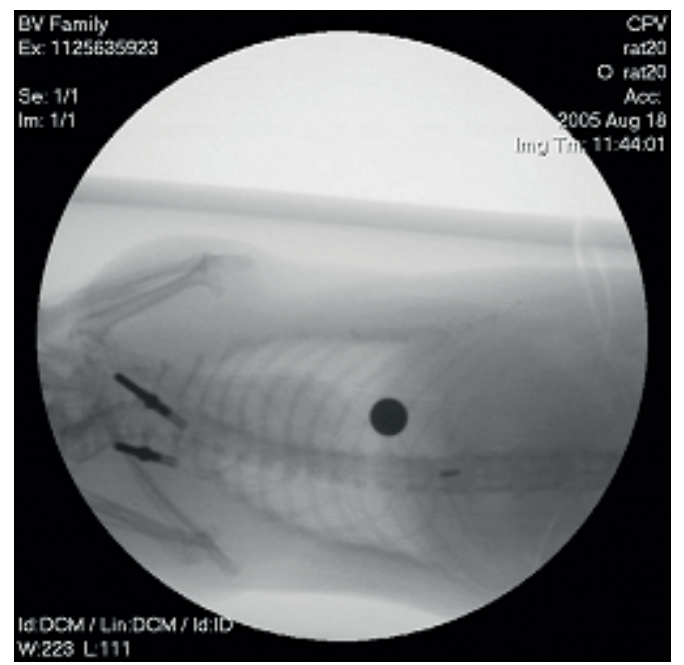

Figure 1: Electrode positions were verified by means of X-ray. X-ray allows identification of vertebrae and ribs. The T11 - T13 vertebrae were identified by counting the ribs of the animal: the $11^{\text {th }}$ rib is rostrally attached to the corpus of the $11^{\text {th }}$ thoracic vertebrae. Here the cathode is positioned at T11 whereas the anode is located adjacent to the vertebral column and placed subcutaneously. Orientation: Cranial to the right and Caudal to the left

\section{Development of allodynia}

For the T $13(n=16)$ and T 11 level $(n=17)$ implanted group pre-nerve lesion baseline withdrawal thresholds were $80.0 \pm 5.2 \mathrm{~g}$ (mean \pm S.E.M.) and $90.6 \pm 5,3 \mathrm{~g}$ (mean \pm S.E.M.) respectively (Fig.2A). In both groups there was a major significant decrease in Paw Withdrawal Threshold (as \% of pre-injury withdrawal threshold or PIPWTH) to $15.3 \pm 5.8 \%$ (mean \pm S.E.M.) $(P<0.0001)$ in the T13 group and to $8.3 \pm 5.2 \%$ of PIPWTH in the T11 group $(\mathrm{P}<0.0001)$ at DPO 2. Over the next days von Frey withdrawal thresholds in both groups remained stable at this level up to SCS at day 16 (DPO 16) (Fig.2A.). No significant difference in von Frey withdrawal thresholds was noted between the T11 and T 13 animals at baseline, DPO2, 7, 14 and 16. In our animals we do not see a very clear and lasting mirror allodynia in the contralateral paw. Also with respect to both the T13 and the T11 group no significant difference in right (contralateral) paw withdrawal thresholds between baseline and start of the SCS was noted : at SCS 0 ; T13, $95 \%$ PIPWTH $(\mathrm{P}<0.62)$ and T11 94\% PIPWTH $(\mathrm{P}<$ 0.49).

\section{Spinal cord stimulation in the total population : T13 versus T11}

In the total population of animals with mechanical allodynia in the T13 group ( $n=16)$ and the T11 group $(n=17)$ SCS resulted in a gradual increase of the withdrawal threshold of the ipsilateral paw (Fig.2B). Pre-SCS withdrawal responses did not differ between both groups and implantation of the lead system did not alter the mechanical sensitivity of the animals. The maximal increase in withdrawal threshold was observed between 15 and 30 min of SCS. Spinal Cord Stimulation at T13 resulted in a major and significant increase in withdrawal thresholds after 15 minutes 
of SCS from pre-stimulation levels of $10.2 \pm 2.8 \%$ (mean \pm S.E.M.) of PIPWTH to 62.6 $\pm 14.5 \%$ (mean \pm S.E.M.) $(P<0.0026)$ (Fig.2B). At the end of SCS ( $t=30$ minutes) at T13 withdrawal threshold were still significantly elevated at $48.5 \pm 11.5 \%$ (mean \pm S.E.M.) of PIPWTH $(P<0.0063)$. After cessation of the SCS at T13 the withdrawal thresholds decreased gradually to $32.9 \pm 11.9 \%$ (mean \pm S.E.M.) of PIPWTH $(\mathrm{P}<0.057)$ at $\mathrm{t}=45$ minutes and $22.7 \pm 10 \%$ (mean \pm S.E.M.) of PIPWTH $(\mathrm{P}<0.24)$ and were no longer significantly different from the pre-stimulation withdrawal thresholds.

The T 11 implanted animals also showed a significant increase after 15 minutes of SCS from $4.8 \pm 0.77 \%$ (mean \pm S.E.M.) to $15.1 \pm 3.2$ (mean \pm S.E.M.) \% of PIPWTH $(\mathrm{P}<0.0061)$ (Fig.2B). At $\mathrm{t}=30$ minutes withdrawal thresholds peaked at $18.4 \pm 4.1 \%$ (mean \pm S.E.M.) of PIPWTH $(\mathrm{P}<0.0051)$. After cessation of SCS at T11 the withdrawal thresholds gradually decreased and were no longer significantly different from prestimulation $(t=0)$ levels.

The AUC of the withdrawal threshold of the SCS-T13 versus the SCS-T11 (between $\mathrm{t}=0$ and $\mathrm{t}=90 \mathrm{~min}$.) shows a significant difference between the groups where the AUC of the SCS-T13 group is significantly greater than the AUC of the SCS-T11 group (SCS-T13: $2321.7 \pm 535.4$ (mean \pm S.E.M.) versus SCS-T11: $866.6 \pm 158.9$ (mean \pm S.E.M.) $(p<0.011)$ (Fig.2C).

\section{Spinal Cord Stimulation: repositioning of electrodes from T12 to T13}

One animal had pre-nerve lesion von Frey withdrawal thresholds below $60 \mathrm{~g}$ (26 g) and was therefore excluded. The other two animals both developed mechanical hypersensitivity as shown by a decrease in withdrawal threshold to von Frey filaments to $3 \%$ of PIPWTH in animal \#1 and 6\% of PIPWTH in animal \#2. Then the electrode was implanted at the T12 vertebral level as verified by X-ray. Thirty minutes of SCS at the T12 level resulted in an increase in the paw withdrawal threshold to $10 \%$ of PIPWTH in animal \#1. After cessation of SCS the paw withdrawal threshold gradually decreased to $3 \%$ of PIPWTH. Repositioning of the electrode to T13 (verified by X-Ray) in the same animal followed by 30 min of SCS resulted in an maximal increase of withdrawal threshold to $25 \%$ PIPWTH. In animal \#2 SCS at the T12 vertebral level did not lead to any increase in paw withdrawal thresholds. However, after re-positioning of the SCS electrode to the T13 level thirty minutes of SCS resulted in an increase in withdrawal treshold to a maximum of $43 \%$ of PIPWTH. Cessation of the SCS after $30 \mathrm{~min}$ lead to a gradual decrease in withdrawal threshold to pre-stimulation levels (6\% of PIPWTH). 


\section{development of allodynia}

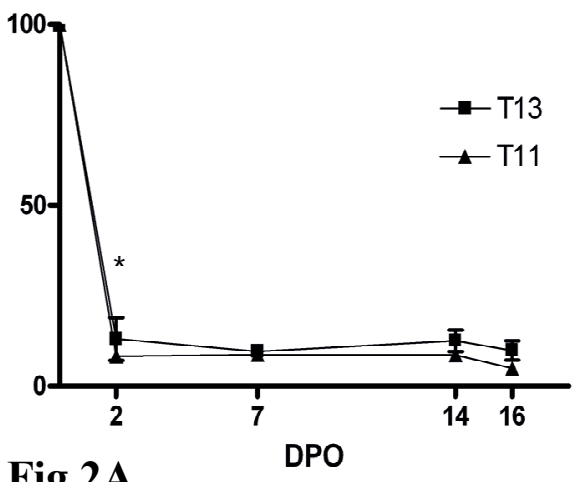

Fig $2 A$

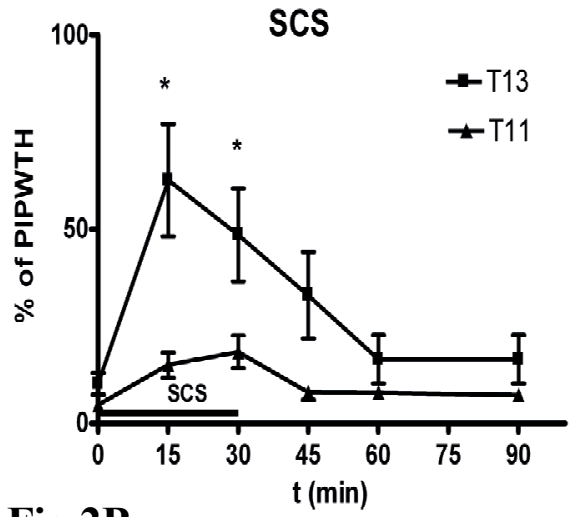

Fig 2B

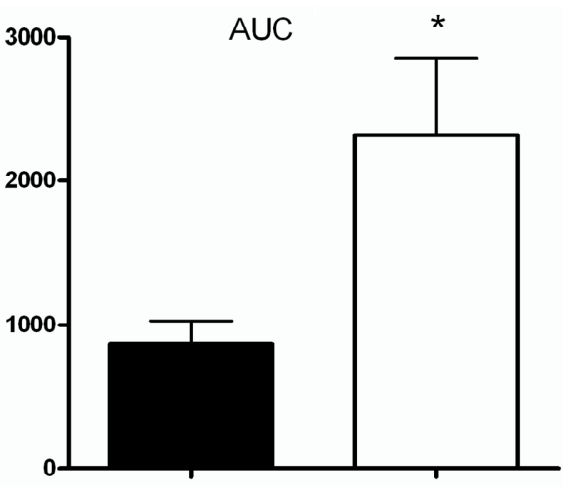

Fig 2C
Figure 2: Total group results. (A) Development of tactile hypersensitivity and implantation of the electrodes at T11 $(n=17)$ or T13 $(n=16)$ after unilateral sciatic nerve ligation. Tactile hypersensitivity is assessed based on changes in paw withdrawal threshold to von Frey hair stimulation. Paw Withdrawal Threshold is presented as $\%$ of Pre-Injury Paw Withdrawal Threshold (PIPWTH) (B) Spinal cord stimulation (SCS) affects the mean foot withdrawal threshold to von Frey hair stimulation in hypersensitive rats both after implantation of electrodes at T13 and T11. The withdrawal threshold increased significantly $\left({ }^{* *} \mathrm{P}<0.005 ; * \mathrm{P}<0.05\right)$ during $\mathrm{SCS}$ being elevated post-SCS for more than one hour after implantation of the electrodes at T13. Implantation of the electrodes at T11 resulted in a significant increase $\left({ }^{*} \mathrm{P}<0.05\right)$ only during the stimulation.

(C) Area under the curve for withdrawal threshold after implantation of electrodes at T13 and T11 shows significant differences ( $\left.{ }^{*}<0.011\right)$. 


\section{Spinal Cord Stimulation and severity of allodynia: T13 versus T11 stimulation}

Based on our previous research (1) SCS-responders were subdivided into three groups related to the severity of allodynia: severe, moderate and mild allodynic rats.

Mild allodynic animals are characterized by a positive response to the filaments with a stiffness corresponding to 8.0-26 (g). After 15 minutes of SCS at T13 $(n=8)$ von Frey withdrawal thresholds showed a major increase from $18.1 \pm 3.8$ (mean \pm S.E.M.) \% of PIPWTH pre-stimulation to $106.0 \pm 16.8 \%$ of PIPWTH (mean \pm S.E.M.) ( $\mathrm{t}$-test compared with $\mathrm{t}=0 ; \mathrm{p}<0.001$ ) and $90.3 \pm 18.8$ (mean \pm S.E.M.) \% of PIPWTH at $\mathrm{t}=30 \mathrm{~min}$. ( $\mathrm{t}$-test compared with $\mathrm{t}=0 ; \mathrm{P}<0.006$ ) (Fig.3A). This implicates a complete return to pre-nerve injury baseline levels. After cessation of the SCS withdrawal thresholds gradually decreased to $59.4 \pm 20.7$ (mean \pm S.E.M.) \% of PIPWTH $(\mathrm{P}<0.087)$ at $45 \mathrm{~min}$ and $38.7 \pm 18.8$ (mean \pm S.E.M.) \% of PIPWTH $(\mathrm{P}<0.31)$ at $t=60$ minutes. The mild allodynic animals stimulated at T11 $(n=4)$ also showed an increase in von Frey withdrawal thresholds from $9.0 \pm 0.5$ (mean \pm S.E.M.) \% of PIPWTH at pre-stimulation to a plateau of $19.2 \pm 3.4$ (mean \pm S.E.M.) \% of PIPWTH after $15 \mathrm{~min}$ and $30 \mathrm{~min}$ SCS. Both increases at $\mathrm{t}=15 \mathrm{~min}$. and $\mathrm{t}=30 \mathrm{~min}$ were statistically significant.

Based on the AUC a significant difference in the von Frey withdrawal response after SCS at T13 and T11 was noted (SCS-T13: $3868.2 \pm 675.8$ (mean \pm S.E.M.) and SCS-T11: $1518.8 \pm 439.4$ (mean \pm S.E.M.) (p<0.039) (Fig.3B).

Moderate allodynic rats were characterized by a response to filaments with a stiffness corresponding to $1.4-6.0(\mathrm{~g})$. In the moderate allodynia group SCS stimulated at the T13 level $(n=5)$ resulted in a maximal increase in withdrawal thresholds after 15 min of stimulation; from $4.3 \pm 0.6$ (mean \pm S.E.M.) $\%$ of PIPWTH at $t=0$ min to $15.9 \pm 4.7$ (mean \pm S.E.M.) at $t=15$ minutes (non significant) (Fig.3A). SCS at the T11 level $(n=10)$ did result in a small but non significant increase in von Frey withdrawal threshold 30 in after stimulation; from $4.3 \pm 0.5$ (mean \pm S.E.M.) \% of PIPWTH to $19.6 \pm 6.5$ (mean \pm S.E.M.) \% of PIPWTH after $t=30 \mathrm{~min}$. No significant difference was noted between the SCS-13 and SCS-T11 moderate allodynic groups based on their AUC (Fig.3B).

Severe allodynic rats (characterized by a positive response to the filaments with a stiffness corresponding to $0.16-1.0$ (g)) did not show a significant increase in von Frey withdrawal threshold during and after the SCS as compared to pre-stimulation thresholds both for the T13 $(n=3)$ and the T11 SCS ( $n=3)$ groups (Fig.3A). No significant difference was noted between the SCS-T13 and SCS-T11 severe allodynic groups based on their AUC (Fig. 3B). 

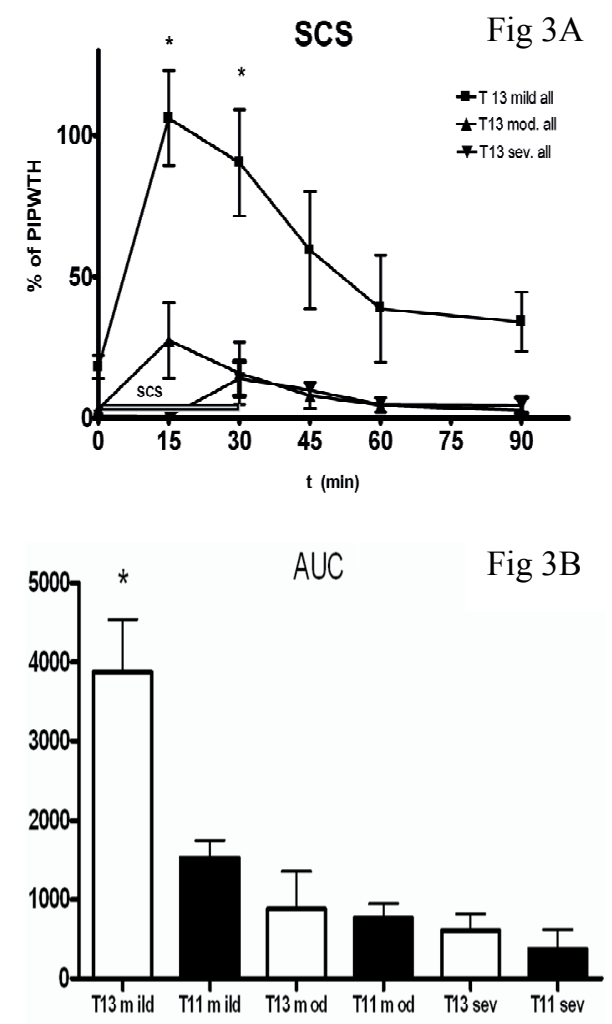

Figure 3: The change in foot withdrawal responses to von Frey stimulation after SCS in relation to severity of allodynia. Paw Withdrawal Threshold is presented as \% of Pre-Injury Paw Withdrawal Threshold (PIPWTH). (A) implantation of electrodes at $\mathrm{T} 13$ resulted in significant changes of withdrawal threshold in mild allodynic rats (* $\mathrm{P}<0.05)$; (B) Areas under the curves where only a significant difference $(*$ $\mathrm{P}<0.039$ ) is noted between the mild allodynic animals stimulated at T13 versus the mild allodynic rats stimulated at $\mathrm{T} 11$.

\section{Discussion}

In the present study it was demonstrated that the spinal level and location of the electrode during SCS stimulation in animals with neuropathic pain significantly affects the pain relieving outcome. This effect which is most obvious in mild allodynic rats. SCS at the T13 level, close to the area where the injured sciatic nerve fibres enter the spinal cord dorsal horn results in a much better pain relieving effect than SCS at more rostral spinal levels, i.e. T11. Furthermore the repositioning of the electrode from $\mathrm{T} 12$ to $\mathrm{T} 13$ results in a better pain relieving outcome which makes additional stimulation of DREZ very unlikely.

Starting from the same experimental neuropathic pain model (56) the question as to what level of implantation and thus level of SCS should be used was often raised. Several vertebral levels of implantation have indeed been used in previous studies as for instance: $T 11(53,54,58,73,84,128)$ and $T 13(1,55,67,71)$. Although the level of implantation is described in many previous studies the actual level was not verified 
by X-ray and thus may be subjected to a certain margin of implantation errors. Incorrect implanted or dislocated electrodes may lead to serious changes with respect to the pain relieving effect of SCS not to mention possible non response to SCS. Inadequate electrode or wire fixation will lead to some degree of technical failure or electrode dislocation in the freely moving animal. To exclude this degree of preventable failure and in line with clinical SCS we use an accurate fixation method to prevent medial-lateral - as well as rostral-caudal movement as the wire of the SCS electrode is secured with histo-acryl tissue glue to the spine processes situated next to the area of burr hole which is exactly in the midline. This fixation combined with X-ray verification (for rats: $(1,67)$; for mice $(103)$. We decided to control for the electrode position both after the implantation procedure as well as immediately after the stimulation procedure. In addition, at macroscopic evaluation of the spinal cord tissue after SCS (71) the localization of the electrodes can easily be verified as a sharply imprinted minimal brown coloured deposition of old blood and fibrin from the implantation operation on the dura mater.

From literature it became clear that both anatomical and electrophysiological principles may explain our findings with respect to the fact that SCS at the T13 level, close of SCS important: in the rat the L3-L5 primary afferents originating from the sciatic nerve are to the area where the injured sciatic nerve fibres enter the spinal cord dorsal horn, is more effective. SCS at T13 results in a much better pain relieving effect than SCS at more rostral spinal levels, i.e. T11. From a conceptual point of view our data strongly point to the fact that SCS of the dorsal columns acts through the modulation of a spinal and not a supraspinal mechanism.

The anatomy of the dorsal column in the rat spinal cord makes the vertebral level localized dorso-lateral within the columns at T13 but rearrange to a more ventro-medial level at T11 (89). Furthermore, the present calculations of the fraction of dorsal column fibres actually stimulated (and depolarized) by SCS is likely not to exceed $1 \%$ of the most superficially (dorsally) located afferents as the ability of the SCS electrode to depolarize DC fibres decreases to the third power of the distance from the electrode $(86,87,96,99,140)$.

Our observations are in line with these anatomical and physiological principles and thus a SCS at T13 results in a more effective pain relief as compared to SCS at more rostral levels (T11). In view of the spinal mechanism of action of SCS in treatment of (experimental) neuropathic pain it is also important to note that in the rat the majority of the ascending dorsal column fibres were found to be lost from the dorsal columns and only $15 \%$ actually reaches cervical levels (89). A segmental mechanism of action is further substantiated by biochemical and pharmacological studies showing significant changes in neurotransmitter contents in the lumbar spinal dorsal horn $(53,58,67,73)$. In view of the segmental mechanism of action of the SCS the severity of allodynia may play an important role. Hypersensitivity and 
allodynia are related to central sensitization. Hypersensitivity is the result of central sensitization induced by neurochemical changes in pain transmission in the dorsal horn of the spinal cord due to an increased release of the excitatory neurotransmitters glutamate and aspartate and at the same time a loss of tonic GABA mediated inhibition (74). SCS causes an increased extracellular GABA-concentration (58) and combined with a decreased extracellular concentration of glutamate $(53,58)$ this results in the suppression of hyperexcitable Wide Dynamic Range Neurons (WDR). The non-response to SCS in severe allodynic rats may be related to a severe form of central neuropathic derangement and may imply a disability to produce appropriate amounts of the inhibitory neurotransmitter GABA, either alone or accompanied by an increased loss of inhibitory interneurons (111). In mild allodynic animals the deranged neurotransmitter balance can significantly be restored by SCS (1), in contrast to the moderate and severe allodynic animals. Then stimulation at T13 which is more optimal location of stimulation of dorsal column fibres that connect to the neuropathologically deranged dorsal horn, results into a significantly improved pain relieving effect as compared to T11 stimulation especially in the mild allodynic animals. The impact of the severity of allodynia and the effect of SCS in pain relief was demonstrated in an experimental model (71) and could be extended into the clinical setting: brush-evoked allodynia was shown to be a significant negative prognostic factor of SCS treatment outcome after 1 year in complex regional pain syndrome type 1 patients (68)

Although the main goal of SCS in treatment of experimental neuropathic pain is to stimulate thick myelinated $A \beta$ fibres in the dorsal columns it cannot be excluded that also incoming dorsal root fibres incuding the $\mathrm{C}$ - and A-delta fibres are directly stimulated through the relatively large-sized experimental electrodes. A recent experimental electrophysiological study further substantiated the possible involvement of the dorsal root as a site of action by which SCS reduces neuropathic pain. SCS at the dorsal column but also at the lumbar dorsal roots attenuated dorsal horn neuronal hyperexcitability in nerve-injured rats (141). Based on these findings it is obvious that in our experimental set-up SCS at the T13, which then directly stimulates the incoming injured dorsal root afferents, results in a more beneficial in pain relieving effect than SCS at T11. On the other hand repositioning of electrodes from T12 to T13 (similar to "paraesthesia steering" in the clinic) resulted in a better pain relief if the electrode is located at T13 as compared to T12. This suggest that additional stimulation of DREZ is very unlikely.

It should, however, be noted that still some pain relief is present when the electrode is located at T11 (or T12 in the repositioning experiment). This finding illustrates that despite the major importance of the localisation of the electrode and the spinal mode of action, still some supraspinal elements may contribute to the pain relieving effect of SCS (65) 
It should be noted that the electrical field of epidural stimulation may spread to nearby tissues via highly conductive cerebrospinal fluid (CSF). For instance the thickness of the CSF may substantially affect the effect of SCS as it is known that if the CSF space increases the threshold current for stimulation of the dorsal columns results in a reduced effectivity of SCS in terms of pain relief (99). In our rat model $(1,66,67,71)$ we use very thin $0.10 \mathrm{~mm}$ platinum- iridium electrodes that were designed to avoid spinal cord compression after placement in the epidural space. It can, however not be excluded that the rat CSF space at T11 is larger compared to T13 and that our electrodes are not thin enough not to compress the CSF space. This then may partly explain the reduced effect of SCS at T11 as compared to T13. In this context it important to note that the use of relatively thick, hand-made electrodes ( $0.25 \mathrm{~mm}$ thick) as previously used in many experimental studies may result in compression of the CSF and thus a increased effectivity of the pain relief despite a non-optimal location of the electrodes (84).

Furthermore it is interesting to note that recent developments in electrode technology allowed the use of multipolar electrodes in the experimental setting. These multipolar leads covered spinal cord segments at vertebral level T10 to T12. The technology of experimental multipolar electrodes is fastly progressing but still facing major limitations. For instance in view of the possible effect of the thickness of these electrodes and compression of the CSF it should be noted that present experimental multipolar leads are relatively thick $(0.35 \mathrm{~mm})(102)$ which is more than three times thicker than plate electrodes used in the present study). This in itself may also considerably affect the outcome.

\section{Conclusion}

SCS of the dorsal columns in treatment of experimental neuropathic pain acts through a segmental spinal site of action. 



\section{Chapter 5 \\ Spinal Cord Stimulation induces c-Fos expression in the Dorsal Horn in Rats with Neuropathic Pain after Partial Sciatic Nerve Injury}

Helwin Smits MD, Maarten V. Kleef MD PhD, Wiel Honig, Job Gerver Msc, Philipp Gobrecht Msc, Elbert. A. J. Joosten PhD. 


\begin{abstract}
Spinal cord stimulation (SCS) is an established treatment for intractable neuropathic pain, especially CRPS-1. The mechanisms of action of SCS have only been partly elucidated and include suppression of the hyperexcitability of the Wide Dynamic Range neurons and a GABA increase in the dorsal horn. In the present study we demonstrate an increase of c-Fos immunoreactive cells in the dorsal horn after SCS, suggesting early cellular activation that may preclude earlier described electrophysiological and biochemical changes in the dorsal horn after SCS. In a rat model of neuropathic pain, allodynia was induced and quantified using the von Frey test. In 11 rats a SCS device was implanted and SCS spinal cord stimulation performed. Withdrawal threshold were measured every 15 minutes up to 90 minutes. A sham group $(n=6)$ also had a SCS device implanted, but did not receive SCS. After SCS the animals were perfused and histology was performed for quantification of c-Fos immunoreactivity in the dorsal horns. We found a significant increase in c-Fos in the SCS group compare to our sham group and control tissue, indicating late cellular activity in the dorsal horn after SCS.
\end{abstract}




\section{Introduction}

Spinal cord stimulation (SCS) is an established clinical treatment for intractable neuropathic pain, especially CRPS-1 (Complex Regional Pain Syndrome type 1), leading to $50 \%$ pain relief in 2/3 of CRPS-1 patients and an increase in Health Related Quality of Life (49). Despite its success, the possible mechanisms of action underlying SCS have only been partially revealed in several experimental studies $(54,58,69,124,126)$. In the present study neuropathic pain was induced through the unilateral ligation of about half of the sciatic nerve at high thigh level as described by Seltzer et al (56). The unilateral ligation is known to result in touch-evoked allodynia and hyperalgesia as assessed using von Frey filaments (56). For experimental studies on SCS the Seltzer injured rats were treated with a miniature system for SCS, described by Meyerson et al (124). In the present study we demonstrate an increase in c-Fos immunoreactive cells in the dorsal horn after SCS, suggesting late cellular activation in the dorsal horn. The gene c-Fos is rapidly and transiently (t1/2 = 15 minutes) (142) expressed in neurons after a wide variety of stimuli (electrical, noxious, chemical and nerve injury) and codes for the production of c- Fos protein (143). The c- Fos protein is involved in a cascade of signal transduction that triggers long-term modulation of spinal nociceptive processes $(144,145)$. c-Fos expression in neuronal cells can be elicited by depolarizing stimuli (144) and was demonstrated to be dependent on the temporal characteristics of the action potential (146). In SCS the electric pulses also generate a pattern of depolarizations moving from the dorsal columns to the dorsal horns and felt by the patient as paresthesias and pain relief. However in experimental SCS the pain relief clearly outlasts the duration of stimulation (126) which suggests a mechanism that lasts longer then the actual electric stimulation. In the present experiment we report an increase in c-Fos in the dorsal horns directly after stimulation SCS as compared to the sham group and control tissue. This suggests that spinal cord stimulation causes early gene regulation that may trigger longer term changes resulting in pain relief during and after SCS

\section{Material and methods}

\section{Animals}

In this study a total of 25 male Sprague-Dawley rats (300-400 g, 12 weeks) were used. The animals were individually housed from week 12 , under standard conditions with a 12-h light/dark cycle (lights off from 18:00-06:00 h) and free access to food and water. All experimental procedures were performed according to the recommendations of the European Commission and protocols were approved by the Committee on Animal Research of the Maastricht University (DEC \# 2005/034). Every attempt was made to minimize the number of animals and their suffering. 


\section{Anesthesia and surgery}

All operations were carried out under sterile conditions and performed under general anesthesia. Induction was performed in a Plexiglas induction chamber using $5 \%$ isoflurane. Anesthesia was maintained with an Inventor $400^{\circ}$ injection system vaporizer (Zevenaar, Holland) with an open mask system using $2.5 \%$ isoflurane in a 1:1 mixture of air and $100 \%$ oxygen at a flow of $250 \mathrm{ml} / \mathrm{min}$. Body temperature was maintained at $37.5{ }^{\circ} \mathrm{C} \pm 0.5^{\circ} \mathrm{C}$ with an automatic heating pad (custom made by the Maastricht University Technical Service using an IR Carell ${ }^{\circledR}$ thermostat (Carell, Manheim, PA, USA)). Analgesic drugs were not administered in order to minimize the risk of interference with the development of allodynia (129)

\section{nerve ligation and assessment of neuropathic pain}

Mechanical allodynia was induced by partial ligation of the sciatic nerve as described earlier by Seltzer et al. (56). Mechanical allodynia was assessed with the "von Frey" test, as described earlier by Smits et al (1). The level of allodynia was determined by testing the withdrawal response to tactile stimuli with von Frey filaments of varying thickness. Before the test the animal was kept in an elevated Plexiglas cage with a wire mesh floor and allowed to adapt for $10 \mathrm{~min}$. Subsequently, von Frey filaments were applied through the wire mesh floor to the midplantar surface of the hind paws until the filament bent slightly. We used 14 touchtest von Frey filaments, numbers 5-16 (North Coast Medical, Inc., Morgan Hill, CA, USA) with a regularly calibrated stiffness corresponding to $0.16,0.40,0.60,1.0,1.4$, $2.0,4.0,6.0,8.0,10,15,26,60$, and $100 \mathrm{~g}$. The filaments are presumed to roughly represent a logarithmic scale of applied force and a linear scale of perceived force (information provided by the manufacturer: North Coast Medical Inc.). The filaments were applied to each hind paw in an order of increasing stiffness and the withdrawal threshold of each individual hind paw was defined as the force (in grams) of the filament that induced three of five positive responses (brisk withdrawal). The withdrawal thresholds were tested after a unilateral partial sciatic nerve ligation of the left hind paw at baseline (pre-nerve lesion) and at 2 days postoperative 2 DPO, 14 DPO and 16 DPO. After testing at day 14 the SCS system was implanted and the animals were allowed to recover for two days before start of stimulation.

\section{Spinal cord stimulation (SCS)}

An electrode was implanted epidurally on the dorsal spinal cord at the location of sciatic nerve entry $(L 4, L 5, L 6)$ at the level of the $13^{\text {th }}$ vertebrae as described earlier by Smits et al. (1). Electrode positions were verified by means of X-ray (Pulsera mobile C-arm, Philips Medical Systems BV, Holland) to control for the accuracy of the implantation procedure. Two days after the implantation the implanted electrodes 
were connected to a Grass S 88 stimulator (Astro-med, Grass, Warwick, RI, USA) fitted with a Grass SIU-5 stimulus insulator and a Grass constant current unit. Motor thresholds (MT) were assessed by gradually increasing the current amplitudes at the following stimulator settings: frequency $2 \mathrm{~Hz}$, pulse width $0.2 \mathrm{~ms}$. When the MT was reached symmetrical lower trunk muscle twitches were observed and the current was measured in $\mathrm{mA}$. The SCS current was then set at $2 / 3$ of the MT, with a frequency of $50 \mathrm{~Hz}$. and pulsewidth was maintained at $0.2 \mathrm{~ms}$. These stimulation parameters correspond closely to the parameters that are used clinically. When the rats were subjected to SCS they showed a minor short (freezing) response, but soon resumed normal behavior. SCS was applied for $30 \mathrm{~min}$ and the withdrawal thresholds to touch of both hind paws were assessed at $t=0,15,30,45,60$ and $90 \mathrm{~min}$. In the sham group $(n=6)$ a SCS device was implanted and the motor threshold was assessed to verify adequate placement of the electrode however, SCS in this group was omitted.

\section{Inclusion criteria}

During the experiment various criteria for inclusion of animals were used. Animals with a pre-nerve lesion baseline of repeated von Frey withdrawal thresholds below $60(\mathrm{~g})$ were not included $(\mathrm{n}=6)$. Furthermore, if the X-ray analysis showed an incorrect position of the electrode (i.e. not at T13) the animal was also not included in the study. Finally, those animals without any elevation in von Frey withdrawal threshold above pre-stimulation threshold after SCS were defined as nonresponders $(n=3)$ to the stimulation protocol and therefore excluded from the analysis.

\section{Immunohistochemistry}

After the SCS session the rats were deeply anaesthetized with intraperitoneal pentobarbital $60 \mathrm{mg} / \mathrm{kg}$ and transcardially perfused with a flush of tyrode solution $(\mathrm{pH}$ $7,40)$ followed by phosphate-buffered $4 \%$ paraformaldehyde. A section of spinal cord from L1-L6 was removed and put in 30\% sucrose solution overnight. The piece of spinal cord was then cut in two halves at the exact place where the centre of the stimulation electrode had been positioned. The material was frozen with liquid isopentane and stored. From each animal at least ten $30 \mu \mathrm{m}$ sections per part were cut using a cryostat. All sections were rinsed in 0.1 M PBS (phosphate buffer solution) for $10 \mathrm{~min}$, using the free-floating method. All incubations have been carried out at room temperature. The sections were incubated with $0.3 \%$ hydrogen peroxide (against endogenous peroxidase) for $30 \mathrm{~min}$ in $0.1 \mathrm{M}$ PBS and subsequently washed in $0.1 \mathrm{M}$ PBS $(3 \times 20 \mathrm{~min})$. A pre-incubation in $0.1 \mathrm{M}$ PBS with $0.1 \%$ bovine serum albumin and $0.2 \%$ Triton-X-100 (PBS-BT) was carried out for 30 min before the overnight incubation with Rabbit-anti-c-Fos (1: 20. 000) (Santa Cruz Biotechnol- 
ogy Inc. SC-052, Santa Cruz, CA) in 0.1 M PBS-BT. After rinsing in 0.1M PBS (3 x 20 $\mathrm{min}$ ), the sections were incubated with Donkey-anti-Rabbit immunoglobulin Gbiotin-conjugated 1:1500 in 0.1M PBS-BT (Jackson ImmunoResearch Laboratories Inc., Westgrove, PA) for $90 \mathrm{~min}$. Then after three washes in $0.1 \mathrm{M}$ PBS, sections were incubated with Vector ABC-Elite (Vector Laboratories, Ltd., Burlingame, CA) (1:800 $\mathrm{M}$ in $0.1 \mathrm{M}$ PBS-BT) for $90 \mathrm{~min}$ followed by a preincubation with DiAminobenzidine-Nickel (DAB-Ni) solution without perhydrol for $10 \mathrm{~min}$. Then sections were incubated with DAB-Ni solution and perhydrol for exactly $10 \mathrm{~min}$. Finally, sections were rinsed three times for $20 \mathrm{~min}$ in $0.1 \mathrm{M}$ PBS and then mounted on gelatin-coated object glasses $(0.5 \%$ gelatin $+0.05 \%$ potassium chrome (III) sulfate), dried overnight at $37^{\circ} \mathrm{C}$. Slices are dehydrated in alcohol series and cleared in Xylol before mounting them in Entellan. The slices received a code allowing blinded counting of c-Fos IR (immunoreactive) cells.

\section{Analysis}

The quantitative analysis of the sections was based on those sections with an optimal anatomic preservation. Quantification of c-Fos IR cells was performed in the dorsal horn area above the position of the central canal, both ipsi- and contralateral. The sections originated from either spinal cord level L1, rostral to the stimulation site (control area) or from the exact position were the centre of the SCS electrode had been positioned at bony level T 13 (stimulation area). Quantification was performed by a blinded and experienced investigator (W.H). This quantification was repeated for intra-observer variability and a second investigator (J.G) also blinded performed a control counting for inter-observer variability. To describe the amount of c-Fos IR cells in the dorsal horn, the total mean number of c-Fos IR cells was calculated in both the contra- and the ipsilateral dorsal horn. It should be emphasised that only those cells with a clear dark intensity were considered as c-Fos immunoreactive and counted. (See fig. 1.)

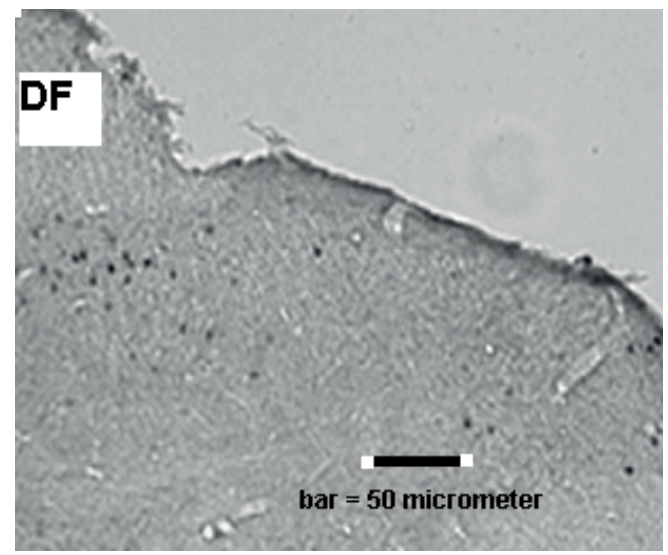

Figure 1: C-Fos IR cells in the dorsal horn of the spinal cord after SCS.

(bar $=50 \mu \mathrm{m}, \mathrm{DF}=$ dorsal funiculus) 


\section{Statistics}

For the stimulation group $(n=11)$ we compared the von Frey withdrawal thresholds during SCS at $\mathrm{t}=30(\mathrm{~min})$ with the pre-stimulus withdrawal threshold at $\mathrm{t}=0(\mathrm{~min})$ using the two sided student's t-test for two groups with equal variance. In all comparisons the level of significance was regarded as $p<0.05$. In the immunohistochemical analysis, the mean number of c-Fos reactive cells in the stimulation group was either compared to the mean number of c-Fos reactive cells in the control tissue or to the mean number of c-Fos reactive cells in the sham operated group $(n=6)$ using a two sided student's t-test for two groups with equal variance and unequal variance respectively. Also in all three groups the mean number of c-Fos IR cells in the ipsi- and contralateral dorsal horns were compared using a two sided student's t-test for equal variance. All results are expressed as the mean \pm SEM.

\section{Results}

\section{Population characteristics}

From our 25 animals a total of 8 rats were excluded. Six animals had pre-nerve lesion withdrawal thresholds below $60(\mathrm{~g})$, one animal was non allodynic after the seltzer procedure and one animal did not respond to SCS. Hence a total of 11 animals in the SCS group and 6 animals in the sham operated group were used for the histological evaluation.

\section{Development of allodynia after partial unilateral sciatic nerve ligation and the effect of SCS}

In the stimulation group $(n=11)$ mean baseline withdrawal threshold was $78.2 \pm 6.3$ (g) (mean \pm SEM). After the Seltzer procedure there was a significant and rapid decrease in withdrawal thresholds and at pre-SCS levels at DPO 16 (two days after implantation of the SCS device) withdrawal thresholds remained at $4.8 \pm 1.5(\mathrm{~g})$ (mean \pm SEM) ( $p<0.001$ compared to baseline), see Fig 1 . SCS resulted in a significantly increased mean withdrawal threshold of $29.0 \pm 10.4$ (g) (mean \pm SEM) at $t=$ thirty minutes ( $p<0.028$, compared to $t=0 \mathrm{~min}$ ). Thirty minutes after the initiation of stimulation SCS was terminated. The mean withdrawal thresholds then decreased slowly to $21.8 \pm 7.5$ (g) (mean \pm SEM) at $\mathrm{t}=45 \mathrm{~min}, 19.7 \pm 6.5(\mathrm{~g})$ (mean \pm SEM) at $\mathrm{t}=60 \mathrm{~min}$ and $10.8 \pm 2.9$ (g) (mean \pm SEM) at $\mathrm{t}=90 \mathrm{~min}$ (Fig 1). In the sham group the average mean pre-injury withdrawal threshold was $86.6 \pm 13.3$ (g) (mean \pm SEM), which decreased significantly after the Seltzer procedure to $7.3 \pm 3.8(\mathrm{~g})$ at DPO 2 and $6.8 \pm 2.7$ (g) (mean \pm SEM) at DPO 16. ( $p<0.0026$ and $p<0.0037$, respectively; t-test compare to pre-injury levels). After determination of the motor threshold in this group, no SCS was performed. 


\section{Number of c-Fos IR cells in SCS, sham and control tissue group (Fig 2)}

In the sham group the total mean number of c-Fos IR cells was $2.5 \pm 1.3$ (mean \pm SEM), there was no significant difference between the total number of c-Fos IR cells in the ipsi- and contralateral dorsal horn. Our results demonstrate that in the control tissue of SCS rats a mean total of $3.3 \pm 0.84$ (mean \pm SEM) c-Fos IR cells is present in the dorsal horn with no difference between the total number of c-Fos IR cells in the ipsi- and contralateral dorsal horn. SCS resulted in a significant increase in the total mean number of $c$-Fos IR cells in the dorsal horn; $8.2 \pm 1.2$ (mean $\pm S E M)(P<$ 0.031 and $p<0.008$, t-test: SCS group compared to control- and sham group respectively) again no difference between the ipsi- and contralateral dorsal horn was found.

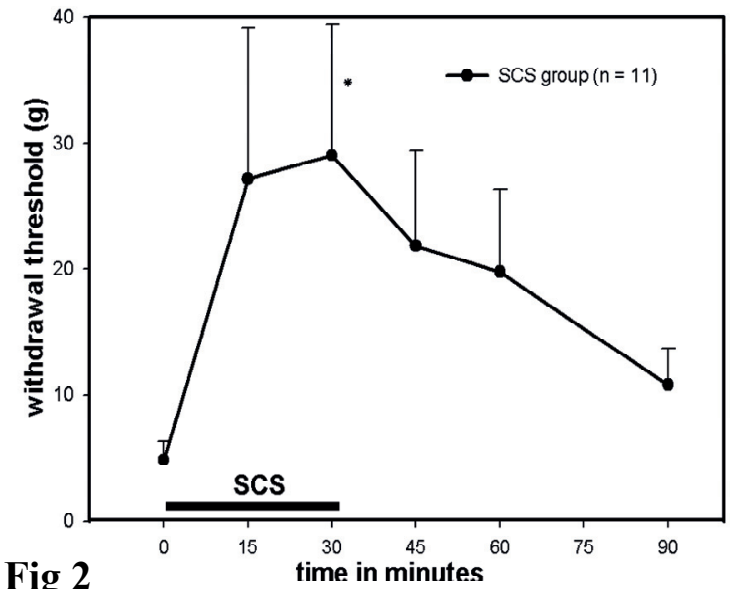

Fig 2

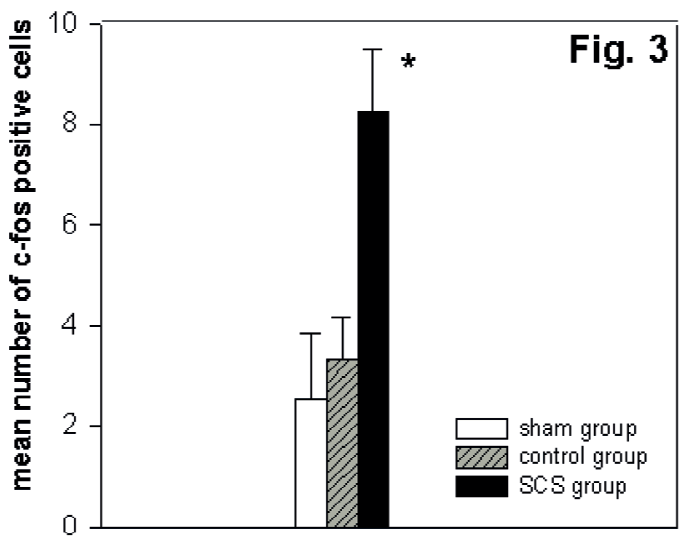

experimental groups
Figure 2: Mean pre-stimulation withdrawal thresholds 16 days after unilateral sciatic nerve ligation and at the start of SCS $(t=0)$ was $4.8 \pm 1.5$ (g) (mean \pm SEM). This threshold increased significantly $\quad(p<0.028)$ during SCS, being elevated post-SCS for more than one hour.

Figure 3: SCS significantly increases the number of c-fos IR cells in the $\mathrm{DH}$ 


\section{Discussion}

In the present experiment we demonstrate for the first time an increased amount of c- Fos IR cells in the dorsal horns of allodynic rats after successful treatment with SCS compared to Sham operated animals and control tissue rostral to the stimulation area. Compared to other studies where c-Fos IR cells were quantified in the dorsal horn, our absolute numbers of c-Fos IR cells were low. We decided to only include the very intensely stained c-Fos IR cells (see Fig 1 ). In many papers the c-Fos immunoreactivity is analyzed without considering the intensity of the staining (147). The latter results in relatively high numbers of c-Fos IR cells, but the question remains if it represents the number of c-Fos IR cells correctly. Many cells stain only slightly above background as also can be noted in our material (Fig. 3). Our method, as also previously described by van Zundert et al (148) is based on the quantification of c-Fos IR cells without any doubt. Moreover our methods are verified for intraand inter- observer variability, which was minimal. Despite the relative low number of c-Fos IR cells significant differences were noted. C-Fos is involved in a cascade of signal transduction that triggers long-term modulation of spinal nociceptive processes $(145,149)$ and known to be increased after peripheral nerve lesions $(144,145,150,151)$. This increase was noted mainly in the first 10 days (147). The early increase of c-Fos immunoreactivity may contain a component of c-Fos expression through surgical injury and inflammation (152). Longer term c-Fos immunoreactivity has been studied extensively in the chronic constriction injury (CCl) model (90) and produced conflicting results. Some authors report a late increase in c-Fos immunoreactivity (>15 days) (145) (153) (154), while others (147) report an increased immunoreactivity only during the first 10 days and an absence of c-Fos IR cells at day 20 . The immediate early gene c-Fos is a marker of cellular activation after a wide variety of stimuli and unfortunately does not inform us about a specific mechanism of action of SCS. However, it indicates cellular activation in the dorsal horns of the spinal cord after continuous high frequency depolarization ( $f=50 \mathrm{~Hz}$ ) with a duration of 30 minutes of the dorsal columns with rectangular electric pulses. The expression of immediate early genes after electrical stimulation of central neurons is a well known fact. From the electric stimulation of cultured mouse dorsal root ganglions it appeared that the temporal aspects of intracellular signalling are critical in controlling neuronal gene expression and the most critical determinant of c-Fos expression was the interval of time between bursts of action potentials (146). It seems that the spatial configuration of action potentials in itself contains information for the cells which are to be depolarized. Historically SCS has been performed as continuous stimulation with frequencies of $50-60 \mathrm{~Hz}$ and a stimulus pulse width of $0.2 \mathrm{~ms}$, with either constant current or constant voltage. A recent experimental SCS study in rats with hyperalgesia after spared nerve injury showed that lower stimulation frequencies of $4 \mathrm{~Hz}$ and $60 \mathrm{~Hz}$ were more effective in increasing with- 
drawal thresholds than higher frequencies of $100-$ and $250 \mathrm{~Hz}$ (102). Also a cummulative reduction in hyperalgesia was noted if multiple SCS sessions were performed (102). It is possible that the effect of SCS can be greatly enhanced by the search to more appropriate stimulation parameters or by adjusting the temporal character of the stimulation; continuous stimulation versus intermittent, or single versus multiple sessions. More extensive experimental and clinical research is needed to explore this.

The question remains if c-Fos plays an important role in the triggering of cells and molecules involved in the proposed mechanisms of action underlying SCS. It has been suggested that inhibition of a hyper-excitable Wide Dynamic Range (WDR) neuron, increased GABA release and the inhibition of the release of excitatory neurotransmitters are involved $(53,55,58,125)$. The earlier experimentally proposed mechanisms of action of SCS like; inhibition of a hyper excitable Wide Dynamic range neuron, increased GABA release and the inhibition of the release of excitatory neurotransmitters $(55,73,125,128)$ may be triggered by the release of the immediate early gene c-Fos. More research should be aimed at the exact location of the cFos expression in the dorsal horn and at the pattern of c-Fos immunoreactivity over the time course of SCS therapy. 


\section{Chapter 6}

\section{Spinal Cord Stimulation for Complex}

Regional Pain Syndrome type-1:

\section{A cohort study with up to twelve years of follow up}

H. Smits, M.A. Kemler, J.W. Geurts,F. Brunner, J.W. Geurts, A.G.H. Kessels, M. van Kleef 


\begin{abstract}
Background

Spinal cord stimulation (SCS) is an effective treatment for intractable CRPS-1 pain that, in nearly $60 \%$ of patients, leads to more than $50 \%$ pain relief for at least 6 months. On the long term the outcome of SCS therapy in terms of pain relief and number of complications data are sparsely available beyond five years of treatment.
\end{abstract}

\title{
Methods
}

Between 1997 and 2008 we included a total of 84 consecutive patients in a prospective study with a follow up of twelve years (median of 5.2 years) to record the effectiveness and complications of SCS. The outcome measures were survival of success as defined by a pain reduction of more than $30 \%$ of the mean VAS score. Furthermore the longevity of the stimulator and the complications requiring reintervention were recorded.

\section{Results}

After 11 years, $40 \%$ of CRPS-1 patients that received SCS experience more than $30 \%$ pain relief. the mean VAS score showed a constant trend. The survival of a functioning SCS system was $65 \%$ after twelve years and the number of re-interventions related to a complication was 72 . Another 44 re-interventions were needed due to battery changes.

\section{Conclusion}

SCS in CRPS-1 patients provides an effective long term pain reduction with an acceptable complication rate. 


\section{Introduction}

Complex Regional Pain syndrome type-1 (CRPS-1) is a painful and debilitating disorder that is highly refractory to conventional pain therapy. In well selected patients with CRPS-1, spinal cord stimulation (SCS) was found to be successful in $56 \%$ of the patients (49). Most cases of spontaneous stabilization or improvement of CRPS-1 appear early on in the disease and later improvement is less common (155). Analysis of the efficacy of therapeutic interventions in long term CRPS-1 cases is therefore of the utmost importance. Information on the effects of SCS in CRPS-1 patients beyond 5 years of treatment is sparsely available (156). Due to the chronic and invasive character of CRPS- 1 data on the outcome, side effects and complications of SCS treatment for CRPS-1 are of vital importance for the future use and development of this therapy. Therefore, we investigated the long term effect of spinal cord stimulation in CRPS-1 patients with respect to the improvement of pain intensity, the complications, and longevity of the spinal cord stimulation system. We will present the up to twelve year follow-up of patients in whom a SCS system was implanted between 1997 and 2007.

\section{Patients and methods}

\section{Selection of patients}

Inclusion in the study was considered in patients if the following CRPS-1 criteria according to the International Association for the Study of Pain (IASP) were met (2), the presence of an initiating noxious event or cause of immobilization, continuous pain, allodynia or hyperalgesia, pain disproportionate to the initiating event; evidence at some time of edema, changes in skin blood flow and/or abnormal sudomotor activity in the region of the pain and absence of conditions that would otherwise account for the degree of pain or dysfunction.

Additional inclusion criteria were: Age between 18-65 years; disease clinically restricted to one extremity; disease duration of at least 6 months; no lasting success with standard therapy over the last six months including physiotherapy, medication, transcutaneous electrical nerve stimulation, and sympathetic blocks and a mean pain intensity of at least $5 \mathrm{~cm}$, as measured on the Visual Analogue Scale (VAS) scale from 0 (no pain) to $10 \mathrm{~cm}$ (very severe pain) according to Jensen and McFarland (157). Exclusion criteria were: the presence of Raynaud disease; the presence or history of neurological abnormalities unrelated to CRPS-1; conditions other than CRPS-1 affecting the function of diseased or contra lateral extremities; blood clotting disturbances or anticoagulation therapy and cardiac pacemaker use. The study protocol complied with the declaration of Helsinki regarding investigations in hu- 
mans and was approved by the medical ethics committee of Maastricht university hospital. All patients gave written informed consent.

\section{Procedure of the Spinal Cord Stimulation}

Patients meeting the inclusion criteria first received a test stimulation trial. The operative procedures concerning the implantation of a test or permanent stimulation system have previously been described in detail (49). The decision to implant a permanent SCS system (Medtronic Itrel $3^{\circledR}$ stimulator and Medtronic Quad ${ }^{\circledR}$ lead) was made if the test stimulation of at least 7 days was successful. In this respect success was defined as a reduction in pain intensity in the last four days of the testing period of at least $50 \%$ in pain intensity as measured by the VAS score or if "much improvement (6 points) was reported on a 7-point global perceived effect scale. Patients who did not meet these criteria did not receive a permanent SCS system and were excluded from this study.

\section{Data collection}

Before trial stimulation gender, age, level of education, baseline VAS scores, pain localization, and the initiating event were recorded.

After trial stimulation VAS pain scores were assessed and after definitive implantation of the SCS system VAS scores, complications and reasons for re-intervention were registered annually.

All SCS related complications that required re-intervention were registered during the study and could be divided in the categories displaced electrode, hardware malfunction, fractured electrode, pulse generator discomfort, pain, superficial infection, deep infection, not specified and inadequate stimulation. Additionally, replacement of empty batteries was recorded.

\section{Statistics}

We defined the SCS therapy as successful when the patient scored a pain reduction of more than $30 \%$, as measured by their VAS scores (158) (159). Failure was defined as less than $30 \%$ pain reduction compared to baseline (pre-SCS) in two consecutive VAS score measurements, Or; one measurement of less than $30 \%$ pain relief compared to baseline if this was the last observation. A once-only peak value of less than $30 \%$ pain relief compared to baseline VAS score was not considered as a failure of SCS as this occurred in several patients that continued to experience $>30 \%$ pain relief after this incidental measurement. Otherwise the second observation of (a VAS score) < $30 \%$ pain relief (compared to baseline) was taken as a failure. The life span of a successful SCS therapy was recorded and plotted in a Kaplan-Meier survival curve. The time curve of the mean VAS scores after one year of follow up was analyzed using a mixed linear regression model. Additionally, the longevity of 
the SCS system was analysed with a Kaplan-Meier plot with failure defined as explantation of the SCS system.

\section{Results}

\section{Study population}

In total 84 patients were included in the study in the period between 1997 and 2007. The duration of follow up ranged from 0.2 to 11.9 years with a median of 5,2 years. The first 24 patients receiving SCS also participated in the RCT of Kemler et al. (49). In figure 1 an overview of the included patients per year is presented. A summary of the population characteristics is presented in table 1.

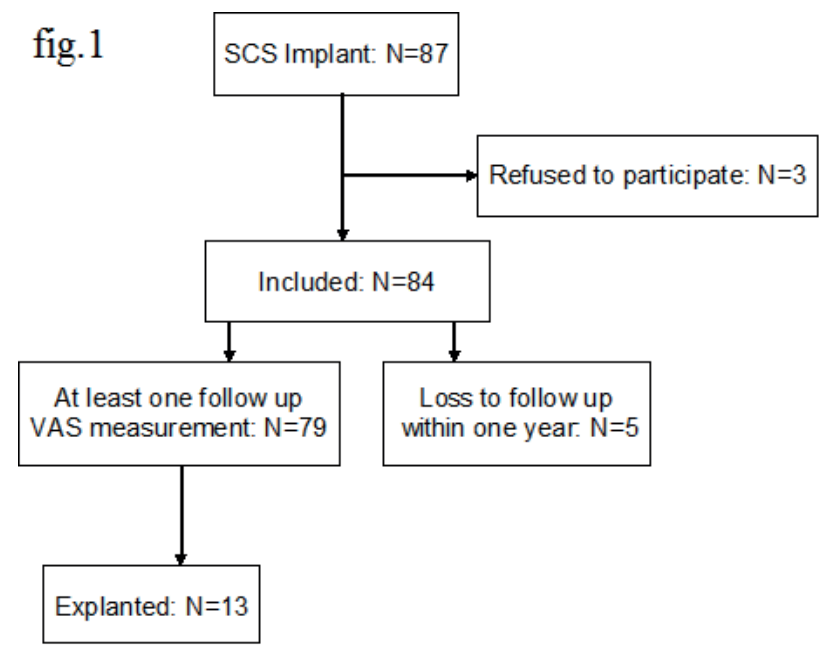

Figure 1: Flowchart of inclusion of patients receiving SCS

\section{Effects on pain}

In figure 2 the mean VAS scores as function of the time is displayed. To examine the trend in time the regression line of the means between the first and twelfth year of follow up is depicted.

The survival curve of more than $30 \%$ decrease in VAS score in figure 3 shows that after 11 years $40 \%$ of the patients experience a pain reduction of more than $30 \%$ 
Table 1: Baseline characteristics of included SCS patients

\begin{tabular}{ll}
\hline $\begin{array}{l}\text { Gender } \\
\text { male }\end{array}$ & $22(26 \%)$ \\
female & $62(74 \%)$ \\
Median age (IQR $\left.{ }^{*}\right)$ & $35(32-46)$ \\
Education & \\
primary & $15(19 \%)$ \\
secondary & $49(55 \%)$ \\
tertiary & $16(29 \%)$ \\
Initiating event & \\
surgery & $29(35 \%)$ \\
trauma & $46(55 \%)$ \\
spontaneous & $9(11 \%)$ \\
Localization & \\
arm affected & $45(54 \%)$ \\
leg affected & $39(46 \%)$ \\
Median VAS score (IRQ & $77(65-87)$ \\
Pain duration until SCS in years (IRQ $)$ & $2.7(1.7-4.9)$ \\
\hline
\end{tabular}

*IQR: InterQuartile Range

\section{3. complications and battery changes}

In the total population a total of 72 complications occurred. The two most frequent complications were; dislocation of the electrode 27 times (23\%) and hardware malfunction 13 times (11\%) (see Table 2). Electrode dislocations occurred in a total of 24 patients and were recurrent in some. Electrode dislocation usually occurred early with a mean failure time of 1.9 years. Hardware malfunction requiring surgical replacement was reported 13 times (11\%) in total.

Battery changes: In total 44 battery changes were performed and in 11 patients batteries had to be changed more than once (see table 2). Other reasons for reinterventions remained under six percent of complications. Infection was suspected clinically in a total of 3 of 75 patients who had their stimulators removed as a result of this. Bacterial cultures remained negative however in all cases. Other complications did not occur in our study population.

The survival time of the system i.e. the time until explantation, is shown in fig 4. At final follow up $65 \%$ of the implants are still implanted. 
Table 2: Number of re-interventions their reasons

\begin{tabular}{lrr}
\hline Replacement of battery $^{*}$ & 44 & \\
Complications & 72 & \\
displaced electrode & 27 \\
hardware malfunction & 13 \\
fractured electrode & 7 \\
pulse generator discomfort & 5 \\
pain & 7 \\
superficial infection & 1 \\
deep infection & 2 \\
inadequate stimulation & 7 \\
not specified & 3 \\
total re-interventions & & \\
\hline
\end{tabular}

${ }^{*} 44$ replacements in 12 patients $1 x$, in 7 patients $2 x$, in 2 patients $3 x$, in 1 patient $4 x$ and in 1 patient $5 x$.

Mean battery life until replacement 4.4 years

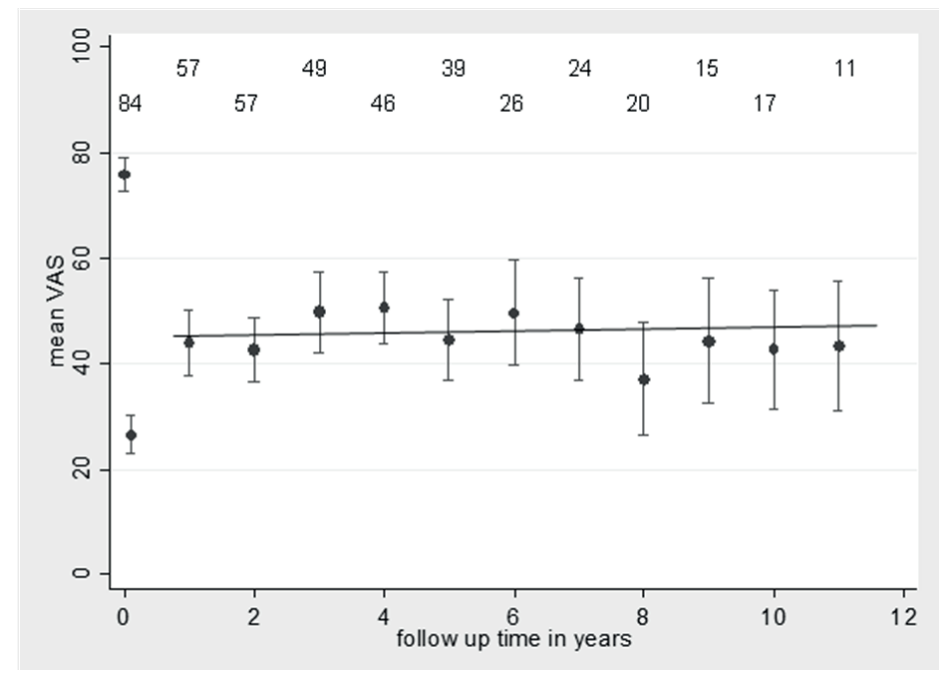

Figure 2: Mean of the VAS score as function of the follow up time with the linear regression line of the last 11 time points (mean_VAS $=44+0.6 *$ follow_up_time in years). The numbers in top of the figure depict the number of observations of varying subsets of patients at the time points 


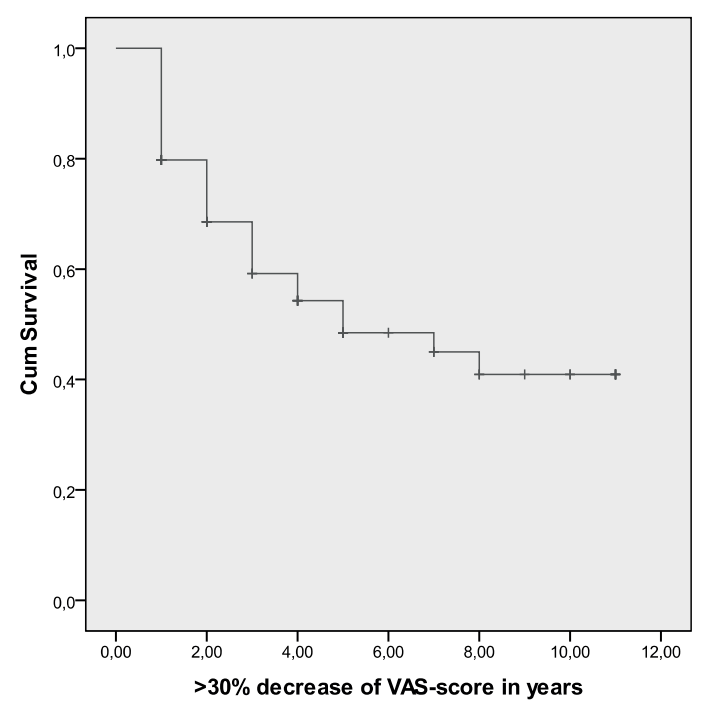

Figure 3: Survival curve of success as defined by $>30 \%$ decrease of the original VAS score

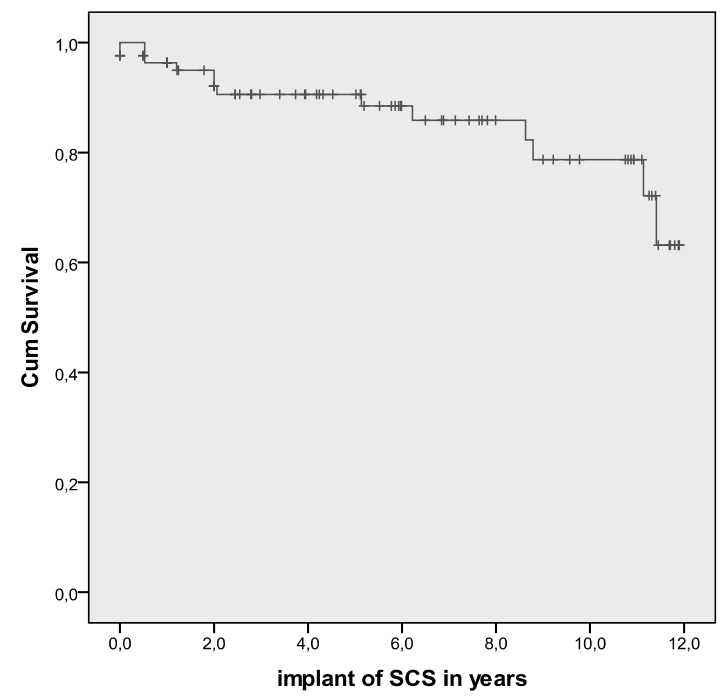

Figure 4: Survival of the implant of the SCS system

\section{Discussion}

This prospective study shows that SCS leads to a long term pain relief as measured by the VAS scores in $40 \%$ of CRPS- 1 patients. The mean VAS score stabilizes (or even decreases slightly) around $60 \%$ of the mean baseline VAS. Major complications like epidural haematomas or infections that were confirmed by bacterial cultures did 
not occur in our study population. The complications reported in this study were relatively minor and could all be solved by small surgical procedures. Although a substantial amount of battery changes was reported, the overall longevity of the SCS system was rather good as after 12 years in $65 \%$ of the patients the SCS system is still functioning.

The prospective nature of data collection increases the level of evidence of this study compared to a previous retrospective long term follow up study on of SCS in CRPS-1 patients. The database used for this study to our knowledge is currently one of the largest ( $n=84$ included patients) and describes the longest follow up period of SCS for CRPS-1 pain reported so far. The results however may still be overestimated as in this study there is no control group to adjust for the natural course.

There is level 3 evidence that SCS is effective for two indications; CRPS- 1 and Failed Back Surgery Syndrome. For CRPS-1 pain SCS results in an initial pain reduction of $>50 \%$ in $56 \%$ of patients and an increase in health-related quality of life (HRQL) (45) For SCS in CRPS-1 patients at two years follow-up, the randomized controlled trial showed a significant $2.1 \mathrm{~cm}$ pain relief in the $S C S$ group versus $0 \mathrm{~cm}$ in the control group (50). However, at five year follow up the pain relieving effect of SCS as compared to the control group had decreased to a level of non-significance after 3 years of follow-up (160). At the end of the five year follow up there is a discrepancy between the reported patient satisfaction and the observation that the pain relieving effect of SCS has decreased to a non-significant level (160) The authors explain that this may have been due to; an increase in the severity of CRPS-1 pain over time, spontaneous recovery of some CRPS-1 patients over time and possibly exaggeration of the initial response to SCS by some patients. At 24 months, the RCT on SCS in failed back surgery syndrome has a similar outcome as the two year follow up of SCS in CRPS-1, $(50,161)$ however no further follow up on FBSS in the form of RCT's have been reported since. Our observation that the mean VAS score stabilizes over time is in line with a recent retrospective 7 year follow up study of SCS in CRPS- 1 patients by Kumar et al ( $n=25$ patients) who also describes long term benefit of this therapy (156). The survival curve of the SCS system indicates that the long term failure rate is relatively low, also indicating an ongoing successful use of the SCS system.

For the long term evaluation of the pain relieving effect of SCS the initial pain relief of at least $50 \%$ which is used initially to decide if a patient will receive a permanent spinal cord stimulator may be too demanding to define relevant pain relief on the long term in patients receiving SCS for CRPS-1. Therefore success was defined as a pain relief of at least $30 \%$ as measured by the VAS score. This is line with the IMMPACT recommendations by Dworkin et al. (158). They define relevant pain relief as $30 \%$ decrease in VAS score as this pain reduction is associated with ratings not requesting any rescue medication. 
SCS for intractable CRPS-1 pain proves to be a valuable therapy with long term efficacy (years) in about $40 \%$ of the patients. Furthermore the test stimulation is successful in $56 \%$ of patients (49). In SCS a substantial number of patients has no benefit from the intervention and clinical predictors of success or failure may improve patient selection and reduce failure rates (68). However, in the present study we did not find any predictors of success or failure of SCS induced pain relief. In future studies effort should be taken to identify clinical predictors and this research requires the formation of large (multi-centre) patient databases of CRPS-1 patients that receive SCS treatment.

As to the complication rate of long term SCS in CRPS-1 we can conclude that we report 72 relatively mild complications that led to surgical revision. Although these procedures are usually minor, one has to realize that they all do increase the risk for infections (162). The overall infection rate in SCS is estimated at $5 \%$ (163). Several large studies did not report any severe complications (e.g. epidural abscesses or haematomas) in SCS for CRPS-1, FBSS as well as in SCS for severe angina pectoris $(160,161,164,165)$. The most frequently reported complication is lead dislocation in around $30 \%$ (24 -37\%) of patients which is in line with our findings. Electrode dislocation is most frequently reported in percutaneously placed leads and several studies indicate that electrode migration is a common and important reasons for failure to maintain long-term pain control with SCS $(166)(167,168)$.

It has to be taken into account that the hardware used in this study is presently outdated which is an inherent problem of long term follow up studies. In order to decrease the frequency of pulse generator replacement, future research should focus on technical advancements of SCS hardware i.e. recharging by electric field induction or long(er) life batteries. Furthermore, research for innovations that prevent lead dislocation is needed especially for percutaneous leads as this may lead to a significant increase in the long term effectiveness of SCS

Spinal cord stimulation in CRPS-1 patients proves to be effective in pain reduction with an acceptable complication rate in the long term. Improvement of the treatment result may be possible by more specific patient selection and advancements in device related technical aspects 
Chapter 7

Summary and general discussion 



\section{General aim of this thesis}

This thesis includes both experimental and clinical work on SCS in chronic neuropathic pain (NNP). In the experimental part of this thesis the first three research questions were explored in an animal model of NPP and SCS. The latter and clinical part of this thesis focuses on the fourth research question.

The following research questions were addressed (see Chapter 1, Introduction page 20) and based on our research we are able to formulate the following answers:

RQ 1. What is the current status and effectiveness of SCS in the treatment of NPP as related to its technical and anatomic aspects?

SCS is a valuable therapy for neuropathic pain in CRPS-1 and FBSS that still has a 40 $\%$ failure rate. In order to reach a possible future enhancement of the effectiveness of SCS therapy a greater understanding of the mechanism(s) and site(s) of action of SCS combined with a science based and effective use of technical aspects of SCS is needed. In Chapter 2 we summarized all data on technical aspects related to SCS as well as the computer modelling related to the physical and mathematical calculations of conductivity and tissue characteristics. From this we concluded that in order to enhance the effectiveness of SCS a combined translational research effort should be taken that is aimed at

1. The search for clinical predictors of a successful outcome of SCS

2. The development of a computer model that integrates new findings on the neurophysiology, biochemistry and circuitry of SCS into a model that can be used to calculate optimal SCS settings.

3. Experimental research in order to further elucidate the mechanisms of SCS.

RQ 2 Are there signs or symptoms of neuropathic pain that may predict the outcome of SCS in terms of pain relief?

In Chapter 3 we report two important observations: Firstly the response to SCS differed with the severity of mechanical allodynia; mildly allodynic rats completely recovered to their baseline pre-injury sensory levels compared to moderate and severe allodynic animals which showed a partial and a non-response respectively. Secondly, mildly allodynic animals reached the optimal response twice as fast as moderately allodynic animals. These findings might be of clinical importance.

\section{RQ 3 Does SCS of the dorsal columns act via a segmental spinal mechanism?}

Our data in Chapters 4 and 5 demonstrate that SCS of the dorsal columns at the level where the injured fibres enter the spinal cord dorsal horn result in a much better pain relieving effect than SCS at more rostral levels. Furthermore, the increased amount of c-Fos IR cells in the dorsal horns of allodynic rats after successful SCS indicates cellular activation at the level of stimulation. From this we conclude 
that SCS in treatment of chronic neuropathic pain acts through a segmental spinal site of action.

RQ 4 What is the long term (twelve year follow-up) effectiveness of SCS in CRPS-1 in terms of pain relief and what are the frequency and nature of long-term complications of this therapy.

SCS for intractable CRPS-1 pain proves to be a valuable therapy with long term efficacy (years) in about $40 \%$ of the patients (Chapter 6 ). Serious complications like infections and epidural haematomas did not occur in our study population. Surgical re-interventions are quite common and mostly comprise of battery changes and lead repositioning after dislocation.

In experimental pain research the selection of an animal model is crucial. The use of animal models in studies on chronic pain has been under debate as translation of the results to the clinical situation if often of limited success (169). The limited progress in translating preclinical findings may have been caused by failure to recognize the limitations of different assessment methods, over interpretation of behavioural responses and failure to acknowledge which neural circuits are involved in the expression of specific behaviours (170). Nevertheless, an experimental animal model can be an important tool to study underlying mechanisms of disease and create or improve therapies, provided the animal model has enough clinical relevancy. It should be taken into account that studies on experimental SCS in (chronic) neuropathic pain require a complex animal model which is basically consisting of two components; firstly, the induction of neuropathic pain and secondly the treatment with SCS. Whereas in our studies we focussed, clinically at the CRPS-1 patients (see Chapter 6) our animal model should meet the requirements needed. CRPS-1 is characterized by sensory abnormalities, vascular abnormalities, oedema and sweating abnormalities and motor and trophic changes (171) (see also Chapter 1). The sensory abnormalities consist of spontaneous (burning) pain that is disproportionate to any inciting event, touch evoked allodynia/hyperalgesia and cold allodynia (172). The development of CRPS-1 is likely to be caused by an aberrant inflammatory response that causes central sensitization which then might contribute to pain. Increased concentrations of the inflammatory mediators interleukine (IL) -6 and IL-1 $\beta$ were reported in spinal fluid of chronic CRPS-1 patients $(172,173)$. In contrast to CRPS-2, CRPS-1 is thought not to be preceded by a nerve lesion. Hence, from a clinical point of view the animal models of sciatic nerve mononeuropathy as they are commonly used in research on SCS are more likely to mimic CRPS-2. Nevertheless experimental neuropathic pain models characterized by the absence of an axotomy of primary sensory neurons in the dorsal root have been described in literature, for instance; the Ventral Root Transection or VRT-model (174). In the VRT-model the ventral root fibres are lesioned which results in an inflammatory response and this 
subsequently affects the uninjured dorsal root sensory afferents. A major limitation of this VRT-model is the bilateral presence of sensory abnormalities in contrast to the clinically observed unilateral symptoms in the CRPS-1 patients (172). Bilateral sensory abnormalities are noted only in very severe CRPS-1 patients (172). Other animal models that have been used to mimic CRPS-1 are the Chronic Post Ischemia Pain model (175), the tibia fracture (176) and the intra-arterial Substance-P injection model (177). Although these models do mimic CRPS- 1 to some extend none of them have yet been tried in combination with SCS. Although some of these models may display symptoms that are similar to the symptoms seen in CRPS- 1 it is important to realize that CRPS-1, as mentioned before, is a multifactorial disease that is probably caused by environmental as well as genetic factors. Mailis and Wade provided evidence for a genetic basis of CRPS-1 (178). In order to provide a possible future animal model of CRPS-1 based on its genetic profile, the use of mice models may be more useful as the mouse genome displays an easy mutagenesis and $95 \%$ of the mouse genome is similar to that in humans $(179,180)$. A functional SCS neuropathic mouse model has already been introduced by Truin et al (103).

Important factors in the choice of an animal model of neuropathic pain (CRPS-1) are reproducibility, time of onset of the pain symptoms and stability and duration of the symptoms over time. With this in mind, the partial ligation of the sciatic nerve or Seltzer model of neuropathic pain has a very good profile as the onset of mechanical allodynia/hyperalgesia is almost immediate (hours), the duration of symptoms is reported to be months and this model is characterized by a response: non-response rate of about 70:30 (56).

In view of the response to experimental nerve injury and development of neuropathic symptoms genetic $(181)$ as well as dietary factors $(133,134)$ have been suggested to play an important role.

Genetic differences in sensitivity to mechanical allodynia in rats is reported (182). However, little is known about the identity of predisposing genes. Recently the gene CACNG2 is shown to significantly affect the susceptibility to chronic pain following nerve injury. CACNG2 encodes for stargazin, a protein intimately involved in the trafficking of glutamatergic AMPA receptors (183). which forms a critical step in the central sensitization process $(23,184)$. With respect to dietary factors mediating the response to nerve injury a study by Shir and co-workers reported that the presence of dietary phyto-estrogens in soy containing food reduced NPP symptoms in rats $(133,134)$. However in our own experiments (Chapters 3,4 and 5) the Seltzer (56) rat model of neuropathic pain resulted in high response rates of mechanical allodynia even in the presence of phyto-estrogens in the food. As explained and discussed in Chapter 3 nerve ligation performed exactly at the site that is described in the paper by Seltzer (56) resulted in a high reproducibility and response rate. 
Then with respect to the second component of our animal model: the SCS, it is important to stress that the Seltzer-model used is known to allow the study of a pain relieving effect of the SCS treatment (see Chapter 1). For example neuropathic pain induced by the Gazelius (185) photochemical sciatic nerve lesion model animals do not respond to SCS therapy without additional administration of intrathecal drugs. A recent experimental neuropathic pain model that also seems to respond poorly to SCS is the spared nerve injury model (SNI) (132). Commonly used successful animal models of mononeuropathy and experimental SCS are the partial ligation of the sciatic nerve (56) but also the Chronic Constriction Injury (CCI) of the sciatic nerve by Bennett and Xie (90). Despite all possible limitations the Seltzer model of chronic neuropathic pain has been shown to be very translational with respect to study and effects of SCS as related to CRPS type 1 patients:

\section{Analogy between experimental and clinical findings in pain relieving effect}

Both in clinical use of SCS in CRPS-1 patients as well as the experimental use of SCS in Seltzer injured animals resulted in similar proportions of responders (2/3) and non-responders (1/3). This is striking because we should take into account that different outcome measurements are used in experimental and clinical evaluation of the pain relieving effects. In the clinical setting pain reduction is measured by the VAS scores which relates to a reduction in spontaneous pain (49). The experimental results refer to a decrease in mechanical allodynia, as measured by an increase in withdrawal threshold to tactile stimuli with the von Frey test.

2. Analogy between experimental and clinical findings in the mechanism underlying non-response to SCS

In various experimental neuropathic pain models, including the partial ligation of the sciatic nerve or Seltzer model, a loss of GABA and its synthesizing enzyme glutamate decarboxylase from the dorsal horn has been reported following nerve injury in rats $(111,184,186,187)$. An important proposed mechanism underlying the effect of SCS in neuropathic pain is the increase of extracellular GABA in the dorsal horn through the facilitation of the GABA-release $(38,58)$. Whereas the responders to SCS were characterized by enhanced GABA-release in the spinal dorsal horn the non-responders did not $(38,73)$. However, when extracellular GABA concentrations in non-responders to SCS were pharmacologically increased through the intrathecal application of sub effective doses of GABA analogues like gabapentin or pregabalin or through the application of the $\mathrm{GABA}_{B}$ receptor agonist baclofen, these animals showed a marked decrease in withdrawal thresholds. Furthermore, intrathecal administration of a sub effective dose of the $\mathrm{GABA}_{\mathrm{A}}$ receptor agonist muscimol did not result in efficient reversal of SCS non-responders into responders $(58,82,128)$. These findings were translated to the clinic where also neuropathic pain patients initially non- 
responding to SCS, did respond to SCS if co-treated with sub effective doses of intrathecally applied baclofen $(83,188,189)$.

3. Analogy between experimental and clinical findings and the prediction of the pain relieving effect SCS related to the severity of allodynia.

Our experimental results as presented in Chapter 3 on the severity of allodynia and effect of SCS on the withdrawal response to tactile stimuli with the von Frey test were translated to the clinic. In Chapter 3 we report an inverse relationship between the severity of mechanical allodynia and the pain relieving effect of SCS: severe allodynic rats do not respond to SCS (Chapter 3; Figure 3). In analogy to this a follow-up study by van Eijs et al. (68) reported that brush evoked allodynia may be a significant negative prognostic factor of SCS treatment outcome after 1 year in chronic CRPS-1 patients.

In this thesis indirect evidence is provided that SCS mainly acts via a segmental spinal mechanism (see Smits et al., 2009; 2011; Chapters 4 and 5). Based on the segmental localization of the electrode (Smits et al., 2011, Chapter 4) as well as the local activation of cells in the dorsal horn of the spinal cord (71) (Chapter 5) it is now clear that in order to obtain a maximal effective pain relief the localization of the electrodes as related to the segmental spinal level is of the utmost importance. However, it should be noted that our data do not completely rule out a possible contribution of supraspinal elements. The latter is based on the fact that also a more rostral localization of the electrodes (i.e T11) still results in some pain relief. There is literature demonstrating the contribution of supraspinal centres based on lesioning of the dorsal columns rostral to the electrode $(65,190)$. It is known that selective lesioning experiments focused at the dorsal columns are very complicated, highly variable in both size and impact and very difficult to qualify in particular at the behavioural level. The additional evidence we provide with respect to a SCS segmental spinal mechanism is a so-called repositioning experiment, which is similar to the paresthesia-steering in the clinic: In the same animal the repositioning of the electrode from T13 to only one segment rostral (T12) already resulted in a significant reduction of the pain relieving effect in our experimental chronic neuropathic pain model.

In view of the positioning of the electrode not only the segmental localization is of the utmost importance but also the medio-lateral positioning. Whereas lateralization of the electrodes is indeed important to SCS-induced analgesia we always examined the medial-lateral position of the electrodes based on X-Ray. It is known that the clinical percutaneous circular leads allow for a lot of medial lateral movement. In our experimental setting we used $1 \mathrm{~mm}$ wide platina-iridium plate electrodes custom made by Medtronics (Bakken Research Centre, Maastricht, The Netherlands). Whereas the dimensions of our leads (width $(1 \mathrm{~mm})$ and thickness 0.10 $\mathrm{mm}$ ) were specifically based on rat spinal cord anatomy and related to the dimen- 
sions of MRI images of the rat spinal cord, the electrodes exactly fit to the space above the dorsal columns without compression of the spinal cord. Macroscopic evaluation of the spinal cord tissue after SCS (as previously mentioned in a histological study (71) (see Chapter 5) allow the localization of the electrodes based on the presence of a sharply imprinted minimal brown coloured deposition of old blood and fibrin from the implantation operation on the dura mater. From this sharply edged print on the dura we concluded that the electrodes were fixed in one place at the midline where they initially are implanted. We suggest that the main reason for this fixation is related to the fact that the electrode is pushed to the arched bone at the dorsal side by the dura and that results in an evenly balanced pressure from the ventral side of the electrode by the underlying CSF underneath which will not allow easy lateral movement. Moreover, in our laboratory the wire of the SCS electrode is secured with histo-acryl tissue glue to the spine processes situated next to the area of burr hole which is exactly in the midline. This also minimizes the medial-lateral as well as the rostral-caudal movement. Although it might be suggested that a CT-scan is needed and results in a conclusive answer on the exact medio-lateral localization of the electrodes we like to indicate that the metal plates of the electrodes will probably produce a scatter in the image due to reflection of the metal, resulting in a deformed image of the electrode and it's direct surroundings. The anterior posterior X-ray we used does mimic the clinical situation in a best possible way. Clinically the medial lateral position of the electrode is adjusted by "paraesthesia steering" which is not possible in animals, although our repositioning experiment (Chapter 4) comes close.

The size of the experimental electrodes related to the dimensions of the spinal cord and spinal roots does not rule out a possible stimulation effect via the dorsal afferents. A electrophysiological study by Guan and colleagues (141) further substantiated this: they studied the effect of a bipolar electrical conditioning stimulation of the dorsal column and lumbar dorsal roots on the response properties of the spinal wide dynamic range (WDR) neurons in rats after $L 5$ spinal nerve injury. Whereas the conditioning stimulation intensity was set at the lowest current that evoked a peak antidromic sciatic $A \beta$-compound action potential without inducing an $A \delta$ - or C-compound action potential they noted that both the dorsal column and the dorsal root conditioning stimulation significantly attenuated WDR neuronal responses to mechanical stimuli in nerve injured rats. Moreover they noted that stimulation of both dorsal columns and dorsal roots inhibited short-term neuronal sensitization (141). These findings indicate that dorsal root stimulation, due to the relatively large size of the electrodes in our rat model, still might form a serious complication in the experimental setting. On the other hand these electrophysiological studies point to the fact that probably also in the clinical setting dorsal root stimulation in treatment of neuropathic pain might be effective as well. In view of the fact that, at least in the experimental setting it cannot be excluded that other 
structures then the dorsal columns are involved and may account for the therapeutic effect of SCS the term dorsal column stimulation may be replaced by the more neutral phrase "spinal axis stimulation", as already suggested earlier (191).

Whereas the WDR cells, located in the deeper dorsal horn layers are, based on the electrophysiological data $(55,141)$ clearly involved in the mechanism underlying SCS induced pain relief, the question still remains how the activation of the large myelinated primary afferents in the dorsal columns finally results in modulating the incoming pain signal. The spinal nociceptive network and the relation with the myelinated primary afferents in the dorsal columns is presented in Chapter 2, Figure 1. In general SCS of the myelinated primary afferents is thought to induce changes at spinal levels as the balance of inhibitory and excitatory neurotransmitters in the dorsal horn is changed (58) (see Chapter 2). In addition several biochemical and pharmacological experimental studies on the mechanisms of action of SCS showed an alteration of the chemical transmission in the spinal dorsal horn $(52,64,72,73)$. There is evidence that the neuropathic pain syndrome, described as peripheral hypersensitivity with allodynia and hyperalgesia, is a result of central sensitization. Central sensitization is a result of neurochemical changes in the pain transmission in the dorsal horn mainly due to an increased release of the excitatory neurotransmitters glutamate and aspartate (74) and at the same time a loss of tonic GABA mediated inhibition. Basically a decreased extracellular concentration of glutamate and at the same time an increased extracellular GABA-concentration have been noted after SCS $(58,72)$ and this results in the suppression of hyperexcitable WDR neurons (55).

The importance of central sensitization and the role of (phosphorylation of) the NMDA receptor during the pain relieving effect of SCS in chronic neuropathic rats was shown in a pharmacological study performed by Michiel Truin (66). It was already known that interference with the process of central sensitization through the antagonism of the NMDA receptor attenuates chronic neuropathic pain. The use of the non-competitive NMDA blocker ketamine has been shown to have analgesic effect on neuropathic pain in both experimental and clinical studies $(110,192,193)$. A combined treatment of SCS and sub-effective dose of i.t. ketamine in nonresponders resulted in a significant reduction of the withdrawal threshold in all previous non-responders to SCS (66).

As indicated in the Figure 1 (Chapter 2) the spinal dorsal horn has been reported to contain a 'silent' circuit between low-threshold afferent fibres and Nociceptive Specific (NS) projection neurons located in lamina I. Although the composition of this circuit has been only partly described excitatory interneurons in the innermost part of lamina II, which express the $\gamma$-isoform of protein kinase C (PKC- $\gamma$ ) are suggested to be important (77) especially because PKC- $\gamma$ interneurons are known to be activated via $A_{\beta}$ fibres signalling (79). In order to develop further insights into the cellular components involved in the SCS mediated modulation of the 
spinal nociceptive network neuro-anatomical studies might add. In a first attempt we studied the number of c-Fos-immunoreactive cells in the dorsal horn immediately after SCS (Chapter 3). Immediate early genes, like c-Fos, have been used as indirect markers of neuronal activity (144) and increases in the number of c-Fos immunoreactive cells have been reported a few hours to a few days after peripheral nerve injury (147). In our study we noted an increase in the number of c-Fos immunoreactive cells in the dorsal horn after SCS at the level of stimulation (Chapter 5). Further characterization of these c-Fos immunoreactive cells is needed. Whereas GABA-ergic inhibitory interneurons are predominantly located in spinal laminae 1-3 (34) and their interference with nociceptive activation of pain signalling neurons has already been pointed out decennia ago (24) a double immunostaining of c-Fos with antibodies directed against the neurotransmitter GABA -is a logical first next step in analyzing the cellular components involved the mechanism underlying SCS mode of action (194).

Another approach might be the in vivo imaging of metabolic activity of cells in neuropathic animals after SCS. Metabolic activity of cells can be visualized using flavoproteins: Mitochondrial oxidized flavoproteins have the ability to absorb blue spectrum photons. This causes an immediate emission of green spectrum photons, whose intensity (detected by a CCD camera) is a direct measure of neuronal metabolic activity.

The autofluorescent flavoprotein imaging (AFI) technique is an optical technique suitable for monitoring metabolic activity in the superficial areas of nervous tissue thereby studying cellular processes in vivo in the cerebellum (195) and cerebral cortex (196). Moreover using the AFI-technique it was possible to demonstrate simultaneously the temporal and spatial propagation of spinal nociceptive activity in vivo in the spinal cord dorsal horn (197). In a pilot study we investigated the effect of spinal cord stimulation on spinal dorsal horn metabolic activity in neuropathic rats in vivo (198). Our preliminary data showed a short-lasting, but strong reduction in AFI intensity relative to baseline in SCS animals, which was not observed in animals that underwent sham stimulation. These observations directly visualize the temporal and spatial extension of spinal hyperexcitability following nerve injury. At present we are analyzing the same animals used for AFI intensity measurements but now, after perfusion fixation, for double immunohistochemical analysis of the immediate early gene c-Fos and the GABA synthesizing enzyme glutamatedecarboxylase (GAD) in the dorsal horn(198).

The latter and clinical part of this thesis focuses on the long term effectiveness of SCS in CRPS-1 patients based on a prospective (cohort) 12 year follow up study of SCS in CRPS-1 patients. Data on the long term outcome beyond five years of treatment of SCS in CRPS-1 patients are sparsely available. As stabilization or improvement of CRPS-1 only occurs early on in the disease, analysis of the efficacy of thera- 
peutic interventions in chronic CRPS-1 patients is therefore of the utmost importance.

\section{Future research}

Both in clinic and in the experimental setting chronic neuropathic pain is characterized by a wide variety of different grades of allodynia. The significant differences in pain relief in groups with different grades of allodynia after SCS in the Seltzer animal model (Chapter 3 ) did initiate a translational clinical study performed by van Eijs et al. (68). This clinical study showed that the presence of brush-evoked allodynia was associated with a lower chance (31\%) of achieving long-term (up to 1 year) pain reduction with SCS treatment, versus an $81 \%$ chance of pain reduction in patients without brush evoked allodynia. Hence the mean and maximum value of brushevoked allodynia proved to be statistically significant predictors of outcome (68). Despite this important translational finding future research should be aimed at the understanding of SCS and the process of central sensitization, as this is believed to be among the most important cellular changes present in chronic neuropathic pain. This then may lead to new clinical predictors on the effect of SCS in pain relief in neuropathic patients and finally result in an increased effectiveness of SCS.

The wide variety of allodynia noted in the experimental model (Chapter 3 ) also shares a lot of analogy to the clinic of CRPS-1 patients and raises the issue of the relatively vague 1994 International Association for the Study of Pain (IASP) definition of CRPS-1 (2). There is a lack of agreement between the different diagnostic sets for CRPS I and this results in different clinical profiles leading to different therapeutic and study populations. This will slow down scientific development and adequate treatment of CRPS-1(199). With respect to our findings on severity of allodynia and the effect of SCS it may be useful to define a gradation of spontaneous pain and positive sensory signs (gain) CRPS-1 and mild, moderate and severe allodynia. It may well be that the presence or absence of allodynia in CRPS-1 patients is based on differences in CRPS-1 pathophysiology, which finally result in a different pain-relief response to SCS. A study by Eberle et al (200) noted differences in clinical symptoms in patients with so-called warm and cold CRPS-1. This might indicate a difference in pathophysiology. Based on this study and the possible different pathophysiological mechanisms in sub-populations of CRPS-1 patients the presence of allodynia and pinprick hyperalgesia, as signs of secondary hyperalgesia, indicate the involvement of central sensitization. A recent study in 692 CRPS-1 patients by de Boer et al (17) demonstrated that the pain and sensory signs in CRPS- 1 tend to increase in time thereby also pointing towards involvement of cellular modulation of the pain signal at different brain levels (central sensitization). From this the authors favour the development of a more mechanism based classification of CRPS- 1 which ultimately should lead to a mechanism based (SCS)-treatment. We have shown that 
the experimental Seltzer model is of use in the understanding of mechanisms underlying SCS and thus can be of great use for future translational research on effectiveness of SCS in neuropathic pain. 


\section{References}



1. Smits H, Ultenius C, Deumens R, Koopmans GC, Honig WM, van Kleef M, Linderoth B, Joosten EA. Effect of spinal cord stimulation in an animal model of neuropathic pain relates to degree of tactile "allodynia". Neuroscience 2006;143:541-6.

2. Merskey HaB, N. Classification of Chronic pain: Descriptions of Chronic Pain Syndromes and definitions of Pain Terms, ed 2.: Seatlle: IASP Press, 1994, pp 40-42

3. Haanpaa M, Attal N, Backonja M, Baron R, Bennett M, Bouhassira D, Cruccu G, Hansson P, Haythornthwaite JA, lannetti GD, Jensen TS, Kauppila T, Nurmikko TJ, Rice AS, Rowbotham M, Serra J, Sommer C, Smith BH, Treede RD. NeuPSIG guidelines on neuropathic pain assessment. Pain;152:1427.

4. Baron R. Mechanisms of disease: neuropathic pain--a clinical perspective. Nat Clin Pract Neurol 2006;2:95-106.

5. Backonja MM, Galer BS. Pain assessment and evaluation of patients who have neuropathic pain. Neurol Clin 1998;16:775-90.

6. Jensen MP, Chodroff MJ, Dworkin RH. The impact of neuropathic pain on health-related quality of life: review and implications. Neurology 2007;68:1178-82.

7. McDermott AM, Toelle TR, Rowbotham DJ, Schaefer CP, Dukes EM. The burden of neuropathic pain: results from a cross-sectional survey. Eur J Pain 2006;10:127-35.

8. Dieleman JP, Kerklaan J, Huygen FJ, Bouma PA, Sturkenboom MC. Incidence rates and treatment of neuropathic pain conditions in the general population. Pain 2008;137:681-8.

9. Harden RN, Bruehl S, Galer BS, Saltz S, Bertram M, Backonja M, Gayles R, Rudin N, Bhugra MK, Stanton-Hicks M. Complex regional pain syndrome: are the IASP diagnostic criteria valid and sufficiently comprehensive? Pain 1999;83:211-9.

10. Bruehl S. An update on the pathophysiology of complex regional pain syndrome. Anesthesiology;113:713-25.

11. Oaklander AL, Rissmiller JG, Gelman LB, Zheng L, Chang Y, Gott R. Evidence of focal small-fiber axonal degeneration in complex regional pain syndrome-I (reflex sympathetic dystrophy). Pain 2006;120:235-43.

12. Wasner G, Schattschneider J, Heckmann K, Maier C, Baron R. Vascular abnormalities in reflex sympathetic dystrophy (CRPS I): mechanisms and diagnostic value. Brain 2001;124:587-99.

13. Wesseldijk F, Huygen FJ, Heijmans-Antonissen C, Niehof SP, Zijlstra FJ. Six years follow-up of the levels of TNF-alpha and IL-6 in patients with complex regional pain syndrome type 1 . Mediators Inflamm 2008;2008:469439.

14. Pleger B, Ragert P, Schwenkreis P, Forster AF, Wilimzig C, Dinse H, Nicolas V, Maier C, Tegenthoff $M$. Patterns of cortical reorganization parallel impaired tactile discrimination and pain intensity in complex regional pain syndrome. Neuroimage 2006;32:503-10.

15. de Rooij AM, Florencia Gosso M, Haasnoot GW, Marinus J, Verduijn W, Claas FH, van den Maagdenberg AM, van Hilten JJ. HLA-B62 and HLA-DQ8 are associated with Complex Regional Pain Syndrome with fixed dystonia. Pain 2009;145:82-5.

16. Bruehl S, Chung OY, Burns JW, Biridepalli S. The association between anger expression and chronic pain intensity: evidence for partial mediation by endogenous opioid dysfunction. Pain 2003;106:317-24.

17. de Boer RD, Marinus J, van Hilten JJ, Huygen FJ, van Eijs F, van Kleef M, Bauer MC, van Gestel M, Zuurmond WW, Perez RS. Distribution of signs and symptoms of Complex Regional Pain Syndrome type I in patients meeting the diagnostic criteria of the International Association for the Study of Pain. Eur J Pain.

18. Perez RS, Kwakkel G, Zuurmond WW, de Lange JJ. Treatment of reflex sympathetic dystrophy (CRPS type 1): a research synthesis of 21 randomized clinical trials. J Pain Symptom Manage 2001;21:51126.

19. Grabow TS, Tella PK, Raja SN. Spinal cord stimulation for complex regional pain syndrome: an evidence-based medicine review of the literature. Clin J Pain 2003;19:371-83.

20. Julius D, Basbaum Al. Molecular mechanisms of nociception. Nature 2001;413:203-10. 
21. Chuang HH, Prescott ED, Kong H, Shields S, Jordt SE, Basbaum Al, Chao MV, Julius D. Bradykinin and nerve growth factor release the capsaicin receptor from PtdIns(4,5)P2-mediated inhibition. Nature 2001;411:957-62.

22. Gold MS, Levine JD, Correa AM. Modulation of TTX-R INa by PKC and PKA and their role in PGE2induced sensitization of rat sensory neurons in vitro. J Neurosci 1998;18:10345-55.

23. Ji RR, Samad TA, Jin SX, Schmoll R, Woolf CJ. p38 MAPK activation by NGF in primary sensory neurons after inflammation increases TRPV1 levels and maintains heat hyperalgesia. Neuron 2002;36:57-68.

24. Melzack R, Wall PD. Pain mechanisms: a new theory. Science 1965;150:971-9.

25. Brodin E, Linderoth B, Gazelius B, Ungerstedt U. In vivo release of substance $P$ in cat dorsal horn studied with microdialysis. Neurosci Lett 1987;76:357-62.

26. Brown DG, Krupp JJ. N-methyl-D-aspartate receptor (NMDA) antagonists as potential pain therapeutics. Curr Top Med Chem 2006;6:749-70.

27. Bleakman D, Alt A, Nisenbaum ES. Glutamate receptors and pain. Semin Cell Dev Biol 2006;17:592604.

28. Qu XX, Cai J, Li MJ, Chi YN, Liao FF, Liu FY, Wan Y, Han JS, Xing GG. Role of the spinal cord NR2Bcontaining NMDA receptors in the development of neuropathic pain. Exp Neurol 2009;215:298307.

29. Ji RR, Kohno T, Moore KA, Woolf CJ. Central sensitization and LTP: do pain and memory share similar mechanisms? Trends Neurosci 2003;26:696-705.

30. Liu XJ, Gingrich JR, Vargas-Caballero M, Dong YN, Sengar A, Beggs S, Wang SH, Ding HK, Frankland PW, Salter MW. Treatment of inflammatory and neuropathic pain by uncoupling Src from the NMDA receptor complex. Nat Med 2008;14:1325-32.

31. Woolf CJ, Salter MW. Neuronal plasticity: increasing the gain in pain. Science 2000;288:1765-9.

32. Hummel M, Strassle B, Miller S, Kaftan E, Whiteside G. Anatomical localization and expression pattern for the NMDA-2D receptor subunit in a rat model of neuropathic pain. Neuroscience 2008;155:492-502.

33. Gardell LR, Vanderah TW, Gardell SE, Wang R, Ossipov MH, Lai J, Porreca F. Enhanced evoked excitatory transmitter release in experimental neuropathy requires descending facilitation. $J$ Neurosci 2003;23:8370-9.

34. Magoul R, Onteniente B, Geffard M, Calas A. Anatomical distribution and ultrastructural organization of the GABAergic system in the rat spinal cord. An immunocytochemical study using anti-GABA antibodies. Neuroscience 1987;20:1001-9.

35. Malan TP, Mata HP, Porreca F. Spinal GABA(A) and GABA(B) receptor pharmacology in a rat model of neuropathic pain. Anesthesiology 2002;96:1161-7.

36. Yaksh TL. Behavioral and autonomic correlates of the tactile evoked allodynia produced by spinal glycine inhibition: effects of modulatory receptor systems and excitatory amino acid antagonists. Pain 1989;37:111-23.

37. Malcangio M, Bowery NG. GABA and its receptors in the spinal cord. Trends Pharmacol Sci 1996;17:457-62.

38. Janssen SP. Differential GABAergic disinhibition during the development of painful peripheral neuropathy. neuroscience 2011;in press.

39. Ren K, Dubner R. Neuron-glia crosstalk gets serious: role in pain hypersensitivity. Curr Opin Anaesthesiol 2008;21:570-9.

40. Milligan ED, Watkins LR. Pathological and protective roles of glia in chronic pain. Nat Rev Neurosci 2009;10:23-36.

41. Moseley GL. Graded motor imagery is effective for long-standing complex regional pain syndrome: a randomised controlled trial. Pain 2004;108:192-8.

42. Sherry DD, Wallace CA, Kelley C, Kidder M, Sapp L. Short- and long-term outcomes of children with complex regional pain syndrome type I treated with exercise therapy. Clin J Pain 1999;15:218-23. 
43. Dworkin RH, O'Connor AB, Audette J, Baron R, Gourlay GK, Haanpaa ML, Kent JL, Krane EJ, Lebel AA, Levy RM, Mackey SC, Mayer J, Miaskowski C, Raja SN, Rice AS, Schmader KE, Stacey B, Stanos S, Treede RD, Turk DC, Walco GA, Wells CD. Recommendations for the pharmacological management of neuropathic pain: an overview and literature update. Mayo Clin Proc;85:S3-14.

44. O'Connor AB, Dworkin RH. Treatment of neuropathic pain: an overview of recent guidelines. Am J Med 2009;122:S22-32.

45. Mailis-Gagnon A, Furlan AD, Sandoval JA, Taylor R. Spinal cord stimulation for chronic pain. Cochrane Database Syst Rev 2004:CD003783.

46. Shealy CN, Taslitz N, Mortimer JT, Becker DP. Electrical inhibition of pain: experimental evaluation. Anesth Analg 1967;46:299-305.

47. Kemler MA, De Vet HC. An objective and standardized test of foot function: normative values and validation in patients with reflex sympathetic dystrophy. Arch Phys Med Rehabil 2000;81:1401-7.

48. North RB, Kidd DH, Lee MS, Piantodosi S. A prospective, randomized study of spinal cord stimulation versus reoperation for failed back surgery syndrome: initial results. Stereotact Funct Neurosurg 1994;62:267-72.

49. Kemler MA, Barendse GA, van Kleef $M$, de Vet HC, Rijks CP, Furnee CA, van den Wildenberg FA. Spinal cord stimulation in patients with chronic reflex sympathetic dystrophy. N Engl J Med 2000;343:618-24.

50. Kemler MA, De Vet HC, Barendse GA, Van Den Wildenberg FA, Van Kleef M. The effect of spinal cord stimulation in patients with chronic reflex sympathetic dystrophy: two years' follow-up of the randomized controlled trial. Ann Neurol 2004;55:13-8.

51. Linderoth B, Stiller CO, O'Connor WT, Hammarstrom G, Ungerstedt U, Brodin E. An animal model for the study of brain transmittor release in response to spinal cord stimulation in the awake, freely moving rat: preliminary results from the periaqueductal grey matter. Acta Neurochir Suppl (Wien) 1993;58:156-60.

52. Linderoth B, Gazelius B, Franck J, Brodin E. Dorsal column stimulation induces release of serotonin and substance $P$ in the cat dorsal horn. Neurosurgery 1992;31:289-96; discussion 96-7.

53. Cui JG, O'Connor WT, Ungerstedt U, Linderoth B, Meyerson BA. Spinal cord stimulation attenuates augmented dorsal horn release of excitatory amino acids in mononeuropathy via a GABAergic mechanism. Pain 1997;73:87-95.

54. Wallin J, Fiska A, Tjolsen A, Linderoth B, Hole K. Spinal cord stimulation inhibits long-term potentiation of spinal wide dynamic range neurons. Brain Res 2003;973:39-43.

55. Yakhnitsa V, Linderoth B, Meyerson BA. Spinal cord stimulation attenuates dorsal horn neuronal hyperexcitability in a rat model of mononeuropathy. Pain 1999;79:223-33.

56. Seltzer Z, Dubner R, Shir Y. A novel behavioral model of neuropathic pain disorders produced in rats by partial sciatic nerve injury. Pain 1990;43:205-18.

57. Dowdall T, Robinson I, Meert TF. Comparison of five different rat models of peripheral nerve injury. Pharmacol Biochem Behav 2005;80:93-108.

58. Cui JG, Linderoth B, Meyerson BA. Effects of spinal cord stimulation on touch-evoked allodynia involve GABAergic mechanisms. An experimental study in the mononeuropathic rat. Pain 1996;66:287-95.

59. Lind G, Meyerson BA, Winter J, Linderoth B. Intrathecal baclofen as adjuvant therapy to enhance the effect of spinal cord stimulation in neuropathic pain: a pilot study. Eur J Pain 2004;8:377-83.

60. Song Z, Ultenius C, Meyerson BA, Linderoth B. Pain relief by spinal cord stimulation involves serotonergic mechanisms: an experimental study in a rat model of mononeuropathy. Pain 2009;147:241-8.

61. Yaksh TL, Wilson PR. Spinal serotonin terminal system mediates antinociception. J Pharmacol Exp Ther 1979;208:446-53.

62. Bardin L, Schmidt J, Alloui A, Eschalier A. Effect of intrathecal administration of serotonin in chronic pain models in rats. Eur J Pharmacol 2000;409:37-43. 
63. Obata H, Saito S, Sasaki M, Goto F. Interactions of 5-HT2 receptor agonists with acetylcholine in spinal analgesic mechanisms in rats with neuropathic pain. Brain Res 2003;965:114-20.

64. Schechtmann G, Song Z, Ultenius C, Meyerson BA, Linderoth B. Cholinergic mechanisms involved in the pain relieving effect of spinal cord stimulation in a model of neuropathy. Pain 2008;139:136-45.

65. El-Khoury C, Hawwa N, Baliki M, Atweh SF, Jabbur SJ, Saade NE. Attenuation of neuropathic pain by segmental and supraspinal activation of the dorsal column system in awake rats. Neuroscience 2002;112:541-53.

66. Truin $M$, Janssen SP, van Kleef $M$, Joosten EA. Successful pain relief in non-responders to spinal cord stimulation: The combined use of ketamine and spinal cord stimulation. Eur J Pain 2011.

67. Truin $M$, van Kleef $M$, Linderoth $B$, Smits $H$, Janssen SP, Joosten EA. Increased efficacy of early spinal cord stimulation in an animal model of neuropathic pain. Eur J Pain;15:111-7.

68. van Eijs F, Smits H, Geurts JW, Kessels AG, Kemler MA, van Kleef M, Joosten EA, Faber CG. Brushevoked allodynia predicts outcome of spinal cord stimulation in complex regional pain syndrome type 1. Eur J Pain 2009;14:164-9.

69. Meyerson BA, Linderoth B. Mechanisms of spinal cord stimulation in neuropathic pain. Neurol Res 2000;22:285-92.

70. Smits $\mathrm{H}$, van Kleef $\mathrm{M}$, Joosten EA. Spinal Cord Stimulation of dorsal columns in a rat model of neuropathic pain: evidence for a segmental spinal mechanism of pain relief.. submitted 2011.

71. Smits H, Kleef MV, Honig W, Gerver J, Gobrecht P, Joosten EA. Spinal cord stimulation induces c-Fos expression in the dorsal horn in rats with neuropathic pain after partial sciatic nerve injury. Neurosci Lett 2008.

72. Cui JG, Linderoth B, Meyerson BA. Incidence of mononeuropathy in rats is influenced by preemptive alteration of spinal excitability. Eur J Pain 1997;1:53-9.

73. Stiller CO, Cui JG, O'Connor WT, Brodin E, Meyerson BA, Linderoth B. Release of gammaaminobutyric acid in the dorsal horn and suppression of tactile allodynia by spinal cord stimulation in mononeuropathic rats. Neurosurgery 1996;39:367-74; discussion 74-5.

74. Woolf CJ, Doubell TP. The pathophysiology of chronic pain--increased sensitivity to low threshold A beta-fibre inputs. Curr Opin Neurobiol 1994;4:525-34.

75. Guan Y, Wacnik PW, Yang F, Carteret AF, Chung CY, Meyer RA, Raja SN. Spinal cord stimulationinduced analgesia: electrical stimulation of dorsal column and dorsal roots attenuates dorsal horn neuronal excitability in neuropathic rats. Anesthesiology;113:1392-405.

76. Cui JG, Sollevi A, Linderoth B, Meyerson BA. Adenosine receptor activation suppresses tactile hypersensitivity and potentiates spinal cord stimulation in mononeuropathic rats. Neurosci Lett 1997;223:173-6.

77. Berger JV. Cellular and molecular insights into neuropathy induced pain hypersensitivity for mechanism based treatment approaches. Submitted 2011.

78. Jaken RJ, Joosten EA, Knuwer M, Miller R, van der Meulen I, Marcus MA, Deumens R. Synaptic plasticity in the substantia gelatinosa in a model of chronic neuropathic pain. Neurosci Lett;469:303.

79. Neumann S, Braz JM, Skinner K, Llewellyn-Smith IJ, Basbaum Al. Innocuous, not noxious, input activates PKCgamma interneurons of the spinal dorsal horn via myelinated afferent fibers. J Neurosci 2008;28:7936-44.

80. Miraucourt LS, Moisset X, Dallel R, Voisin DL. Glycine inhibitory dysfunction induces a selectively dynamic, morphine-resistant, and neurokinin 1 receptor- independent mechanical allodynia. J Neurosci 2009;29:2519-27.

81. Eaton MJ, Martinez MA, Karmally S. A single intrathecal injection of GABA permanently reverses neuropathic pain after nerve injury. Brain Res 1999;835:334-9.

82. Cui JG, Meyerson BA, Sollevi A, Linderoth B. Effect of spinal cord stimulation on tactile hypersensitivity in mononeuropathic rats is potentiated by simultaneous GABA(B) and adenosine receptor activation. Neurosci Lett 1998;247:183-6. 
83. Lind G, Schechtmann G, Winter J, Meyerson BA, Linderoth B. Baclofen-enhanced spinal cord stimulation and intrathecal baclofen alone for neuropathic pain: Long-term outcome of a pilot study. Eur J Pain 2008;12:132-6.

84. Schechtmann G, Wallin J, Meyerson BA, Linderoth B. Intrathecal clonidine potentiates suppression of tactile hypersensitivity by spinal cord stimulation in a model of neuropathy. Anesth Analg 2004;99:135-9.

85. Ossipov MH, Bian D, Malan TP, Jr., Lai J, Porreca F. Lack of involvement of capsaicin-sensitive primary afferents in nerve-ligation injury induced tactile allodynia in rats. Pain 1999;79:127-33.

86. Holsheimer J, Barolat G, Struijk JJ, He J. Significance of the spinal cord position in spinal cord stimulation. Acta Neurochir Suppl 1995;64:119-24.

87. Holsheimer J. Computer modelling of spinal cord stimulation and its contribution to therapeutic efficacy. Spinal Cord 1998;36:531-40.

88. Schunke m. kopf und neuroanatomie Prometheus: Thieme Verlag, 2006.

89. Smith KJ, Bennett BJ. Topographic and quantitative description of rat dorsal column fibres arising from the lumbar dorsal roots. J Anat 1987;153:203-15.

90. Bennett GJ, Xie YK. A peripheral mononeuropathy in rat that produces disorders of pain sensation like those seen in man. Pain 1988;33:87-107.

91. Willis WD. Sensory Mechanisms of the Spinal Cord New YorkWilliam D. Willis Springer, 1991:590.

92. Holsheimer J, den Boer JA, Struijk JJ, Rozeboom AR. MR assessment of the normal position of the spinal cord in the spinal canal. AJNR Am J Neuroradiol 1994;15:951-9.

93. McNeal DR. Analysis of a model for excitation of myelinated nerve. IEEE Trans Biomed Eng 1976;23:329-37.

94. Rattay F. Analysis of models for external stimulation of axons. IEEE Trans Biomed Eng 1986;33:9747.

95. Ranck JB, Jr. Which elements are excited in electrical stimulation of mammalian central nervous system: a review. Brain Res 1975;98:417-40.

96. Holsheimer J. Which Neuronal Elements are Activated Directly by Spinal Cord Stimulation neuromodulation 2002; Volume 51 - 66.

97. Feirabend HK, Choufoer H, Ploeger S, Holsheimer J, van Gool JD. Morphometry of human superficial dorsal and dorsolateral column fibres: significance to spinal cord stimulation. Brain 2002;125:113749.

98. Simpson BA. Pain research and clinical management; electrical stimulation and the release of pain Spinal cord and peripheral nerve stimulation: technical aspects Elsevier, 2003:267.

99. Holsheimer J. Effects of Electrode Positioning on Perception Threshold and Paresthesia Coverage in Spinal Cord Stimulation. Neuromodulation 2007;10:34-41.

100. Holsheimer J, Wesselink WA. Effect of anode-cathode configuration on paresthesia coverage in spinal cord stimulation. Neurosurgery 1997;41:654-9; discussion 9-60.

101. Villavicencio AT, Leveque JC, Rubin L, Bulsara K, Gorecki JP. Laminectomy versus percutaneous electrode placement for spinal cord stimulation. Neurosurgery 2000;46:399-405; discussion -6.

102. Maeda Y, Wacnik PW, Sluka KA. Low frequencies, but not high frequencies of bi-polar spinal cord stimulation reduce cutaneous and muscle hyperalgesia induced by nerve injury. Pain 2008;138:14352.

103. Truin $M$, van Kleef $M$, Verboeket $Y$, Deumens $R$, Honig W, Joosten EA. The effect of Spinal Cord Stimulation in mice with chronic neuropathic pain after partial ligation of the sciatic nerve. Pain 2009;145:312-8.

104. Holsheimer J, Dijkstra EA, Demeulemeester H, Nuttin B. Chronaxie calculated from current-duration and voltage-duration data. J Neurosci Methods 2000;97:45-50.

105. North RB. Spinal cord Stimulation With Interleaved Pulses: A Randomized, Controlled Trial. Neuromodulation 2007;10:349-57.

106. Alo KM. computer assisted and patientinteractive programming of dual octrode spinal cord stimulation in the treatment of chronic pain. Neuromodulation 1998;1:30-45. 
107. Lisman JE. Bursts as a unit of neural information: making unreliable synapses reliable. Trends Neurosci 1997;20:38-43.

108. De Ridder D, Vanneste S, Plazier M, van der Loo E, Menovsky T. Burst spinal cord stimulation: toward paresthesia-free pain suppression. Neurosurgery 2010;66:986-90.

109. Williams KA, Korto K, Cohen SP. Spinal cord stimulation: "neural switch" in complex regional pain syndrome type I. Pain Med 2009;10:762-6.

110. Schwartzman RJ, Erwin KL, Alexander GM. The natural history of complex regional pain syndrome. Clin J Pain 2009;25:273-80.

111. Eaton MJ, Plunkett JA, Karmally S, Martinez MA, Montanez K. Changes in GAD- and GABA-immunoreactivity in the spinal dorsal horn after peripheral nerve injury and promotion of recovery by lumbar transplant of immortalized serotonergic precursors. J Chem Neuroanat 1998;16:57-72.

112. McCreery DB, Agnew WF, Yuen TG, Bullara L. Charge density and charge per phase as cofactors in neural injury induced by electrical stimulation. IEEE Trans Biomed Eng 1990;37:996-1001.

113. Barolat G, Zeme S, Ketcik B. Multifactorial analysis of epidural spinal cord stimulation. Stereotact Funct Neurosurg 1991;56:77-103.

114. North RB, Ewend MG, Lawton MT, Piantadosi S. Spinal cord stimulation for chronic, intractable pain: superiority of "multi-channel" devices. Pain 1991;44:119-30.

115. Koole P, Holsheimer J, Struijk JJ, Verloop AJ. Recruitment characteristics of nerve fascicles stimulated by a multigroove electrode. IEEE Trans Rehabil Eng 1997;5:40-50.

116. Alo KM, Holsheimer J. New trends in neuromodulation for the management of neuropathic pain. Neurosurgery 2002;50:690-703; discussion -4.

117. Barolat G. Spinal cord stimulation for chronic pain management. Arch Med Res 2000;31:258-62.

118. North RB. Spinal cord stimulation for intractable pain: long-term follow-up. J Spinal Disord 1990;3:356-61.

119. Kumar DV, Nighorn A, St John PA. Role of Nova-1 in regulating alpha2N, a novel glycine receptor splice variant, in developing spinal cord neurons. J Neurobiol 2002;52:156-65.

120. North R, Linderoth B. spinal cord stimulation for chronic pain. In: Schmideck H, Roberts D eds. operative surgical techniques. Philadelphia: Saunders Elsevier, 2006.

121. Bruehl S, Harden RN, Galer BS, Saltz S, Bertram M, Backonja M, Gayles R, Rudin N, Bhugra MK, Stanton-Hicks M. External validation of IASP diagnostic criteria for Complex Regional Pain Syndrome and proposed research diagnostic criteria. International Association for the Study of Pain. Pain 1999;81:147-54.

122. Harden RN. Complex regional pain syndrome. Br J Anaesth 2001;87:99-106.

123. Harke H, Gretenkort P, Ladleif HU, Rahman S. Spinal cord stimulation in sympathetically maintained complex regional pain syndrome type I with severe disability. A prospective clinical study. Eur J Pain 2005;9:363-73.

124. Meyerson BA, Herregodts $P$, Linderoth $B$, Ren B. An experimental animal model of spinal cord stimulation for pain. Stereotact Funct Neurosurg 1994;62:256-62.

125. Linderoth B, Stiller CO, Gunasekera L, O'Connor WT, Ungerstedt U, Brodin E. Gamma-aminobutyric acid is released in the dorsal horn by electrical spinal cord stimulation: an in vivo microdialysis study in the rat. Neurosurgery 1994;34:484-8; discussion 8-9.

126. Meyerson BA, Ren B, Herregodts P, Linderoth B. Spinal cord stimulation in animal models of mononeuropathy: effects on the withdrawal response and the flexor reflex. Pain 1995;61:229-43.

127. Meyerson BA, Cui JG, Yakhnitsa V, Sollevi A, Segerdahl M, Stiller CO, O'Connor WT, Linderoth B. Modulation of spinal pain mechanisms by spinal cord stimulation and the potential role of adjuvant pharmacotherapy. Stereotact Funct Neurosurg 1997;68:129-40.

128. Wallin J, Cui JG, Yakhnitsa V, Schechtmann G, Meyerson BA, Linderoth B. Gabapentin and pregabalin suppress tactile allodynia and potentiate spinal cord stimulation in a model of neuropathy. Eur J Pain 2002;6:261-72.

129. Benrath J, Brechtel C, Martin E, Sandkuhler J. Low doses of fentanyl block central sensitization in the rat spinal cord in vivo. Anesthesiology 2004;100:1545-51. 
130. Schmalbruch H. Fiber composition of the rat sciatic nerve. Anat Rec 1986;215:71-81.

131. Decosterd I, Woolf CJ. Spared nerve injury: an animal model of persistent peripheral neuropathic pain. Pain 2000;87:149-58.

132. Li D, Yang H, Meyerson BA, Linderoth B. Response to spinal cord stimulation in variants of the spared nerve injury pain model. Neurosci Lett 2006;400:115-20.

133. Shir Y, Sheth R, Campbell JN, Raja SN, Seltzer Z. Soy-containing diet suppresses chronic neuropathic sensory disorders in rats. Anesth Analg 2001;92:1029-34.

134. Shir Y, Campbell JN, Raja SN, Seltzer Z. The correlation between dietary soy phytoestrogens and neuropathic pain behavior in rats after partial denervation. Anesth Analg 2002;94:421-6, table of contents.

135. Kumar K, Malik S, Demeria D. Treatment of chronic pain with spinal cord stimulation versus alternative therapies: cost-effectiveness analysis. Neurosurgery 2002;51:106-15; discussion 15-6.

136. Kemler MA, Reulen JP, Barendse GA, van Kleef M, de Vet HC, van den Wildenberg FA. Impact of spinal cord stimulation on sensory characteristics in complex regional pain syndrome type I: a randomized trial. Anesthesiology 2001;95:72-80.

137. Shealy CN, Mortimer JT, Reswick JB. Electrical inhibition of pain by stimulation of the dorsal columns: preliminary clinical report. Anesth Analg 1967;46:489-91.

138. Kemler MA, de Vet HC, Barendse GA, van den Wildenberg FA, van Kleef M. Spinal cord stimulation for chronic reflex sympathetic dystrophy--five-year follow-up. N Engl J Med 2006;354:2394-6.

139. Linderoth B, Stiller CO, Gunasekera L, O'Connor WT, Franck J, Gazelius B, Brodin E. Release of neurotransmitters in the CNS by spinal cord stimulation: survey of present state of knowledge and recent experimental studies. Stereotact Funct Neurosurg 1993;61:157-70.

140. Holsheimer J, Nuttin B, King GW, Wesselink WA, Gybels JM, de Sutter P. Clinical evaluation of paresthesia steering with a new system for spinal cord stimulation. Neurosurgery 1998;42:541-7; discussion 7-9.

141. Guan Y, Wacnik PW, Yang F, Carteret AF, Chung CY, Meyer RA, Raja SN. Spinal cord stimulationinduced analgesia: electrical stimulation of dorsal column and dorsal roots attenuates dorsal horn neuronal excitability in neuropathic rats. Anesthesiology 2010;113:1392-405.

142. Sheng HZ, Fields RD, Nelson PG. Specific regulation of immediate early genes by patterned neuronal activity. J Neurosci Res 1993;35:459-67.

143. Hughes $P$, Dragunow $M$. Induction of immediate-early genes and the control of neurotransmitterregulated gene expression within the nervous system. Pharmacol Rev 1995;47:133-78.

144. Hunt SP, Pini A, Evan G. Induction of c-fos-like protein in spinal cord neurons following sensory stimulation. Nature 1987;328:632-4.

145. Kajander KC, Madsen AM, ladarola MJ, Draisci G, Wakisaka S. Fos-like immunoreactivity increases in the lumbar spinal cord following a chronic constriction injury to the sciatic nerve of rat. Neurosci Lett 1996;206:9-12.

146. Fields RD, Eshete F, Stevens B, Itoh K. Action potential-dependent regulation of gene expression: temporal specificity in ca2+, cAMP-responsive element binding proteins, and mitogen-activated protein kinase signaling. J Neurosci 1997;17:7252-66.

147. Catheline G, Le Guen S, Honore P, Besson JM. Are there long-term changes in the basal or evoked Fos expression in the dorsal horn of the spinal cord of the mononeuropathic rat? Pain 1999;80:34757.

148. Van Zundert J, de Louw AJ, Joosten EA, Kessels AG, Honig W, Dederen PJ, Veening JG, Vles JS, van Kleef $\mathrm{M}$. Pulsed and continuous radiofrequency current adjacent to the cervical dorsal root ganglion of the rat induces late cellular activity in the dorsal horn. Anesthesiology 2005;102:125-31.

149. Hughes $P$, Lawlor $P$, Dragunow $M$. Basal expression of Fos, Fos-related, Jun, and Krox 24 proteins in rat hippocampus. Brain Res Mol Brain Res 1992;13:355-7.

150. Chi SI, Levine JD, Basbaum Al. Effects of injury discharge on the persistent expression of spinal cord fos-like immunoreactivity produced by sciatic nerve transection in the rat. Brain Res 1993;617:2204. 
151. Park KA, Sohn, M.K., Chung K.S. and lee, W.T. the changes of neurotransmitter and c-Fos protein in experimental neuropathic rat model Soc Neurosci Abstr 1997;599:1532.

152. Yamazaki Y, Maeda T, Someya G, Wakisaka S. Temporal and spatial distribution of Fos protein in the lumbar spinal dorsal horn neurons in the rat with chronic constriction injury to the sciatic nerve. Brain Res 2001;914:106-14.

153. Molander C, Hongpaisan J, Grant G. Changing pattern of c-FOS expression in spinal cord neurons after electrical stimulation of the chronically injured sciatic nerve in the rat. Neuroscience 1992;50:223-36.

154. Molander C, Hongpaisan J, Persson JK. Distribution of c-fos expressing dorsal horn neurons after electrical stimulation of low threshold sensory fibers in the chronically injured sciatic nerve. Brain Res 1994;644:74-82.

155. de Mos M, Huygen FJ, van der Hoeven-Borgman M, Dieleman JP, Ch Stricker BH, Sturkenboom MC. Outcome of the complex regional pain syndrome. Clin J Pain 2009;25:590-7.

156. Kumar K, Rizvi S, Bnurs SB. Spinal cord stimulation is effective in management of complex regional pain syndrome I: fact or fiction. Neurosurgery;69:566-80.

157. Jensen MP, McFarland CA. Increasing the reliability and validity of pain intensity measurement in chronic pain patients. Pain 1993;55:195-203.

158. Dworkin RH, Turk DC, Wyrwich KW, Beaton D, Cleeland CS, Farrar JT, Haythornthwaite JA, Jensen MP, Kerns RD, Ader DN, Brandenburg N, Burke LB, Cella D, Chandler J, Cowan P, Dimitrova R, Dionne R, Hertz S, Jadad AR, Katz NP, Kehlet H, Kramer LD, Manning DC, McCormick C, McDermott MP, McQuay HJ, Patel S, Porter L, Quessy S, Rappaport BA, Rauschkolb C, Revicki DA, Rothman M, Schmader KE, Stacey BR, Stauffer JW, von Stein T, White RE, Witter J, Zavisic S. Interpreting the clinical importance of treatment outcomes in chronic pain clinical trials: IMMPACT recommendations. J Pain 2008;9:105-21.

159. Forouzanfar $T$, Weber WE, Kemler $M$, van Kleef $M$. What is a meaningful pain reduction in patients with complex regional pain syndrome type 1? Clin J Pain 2003;19:281-5.

160. Kemler MA, de Vet HC, Barendse GA, van den Wildenberg FA, van Kleef M. Effect of spinal cord stimulation for chronic complex regional pain syndrome Type I: five-year final follow-up of patients in a randomized controlled trial. J Neurosurg 2008;108:292-8.

161. Kumar K, Taylor RS, Jacques L, Eldabe S, Meglio M, Molet J, Thomson S, O'Callaghan J, Eisenberg E, Milbouw G, Buchser E, Fortini G, Richardson J, North RB. The effects of spinal cord stimulation in neuropathic pain are sustained: a 24-month follow-up of the prospective randomized controlled multicenter trial of the effectiveness of spinal cord stimulation. Neurosurgery 2008;63:762-70; discussion 70.

162. Rosenow JM, Stanton-Hicks M, Rezai AR, Henderson JM. Failure modes of spinal cord stimulation hardware. J Neurosurg Spine 2006;5:183-90.

163. Woods DM, Hayek SM, Bedder M. Complications of neurostimulation. Techniques in Regional Anesthesia and Pain Management 2007;11.

164. Andrell P, Ekre O, Eliasson T, Blomstrand C, Borjesson M, Nilsson M, Mannheimer C. Costeffectiveness of spinal cord stimulation versus coronary artery bypass grafting in patients with severe angina pectoris--long-term results from the ESBY study. Cardiology 2003;99:20-4.

165. Borjesson M, Andrell $P$, Lundberg D, Mannheimer C. Spinal cord stimulation in severe angina pectoris--a systematic review based on the Swedish Council on Technology assessment in health care report on long-standing pain. Pain 2008;140:501-8.

166. Cameron T. Safety and efficacy of spinal cord stimulation for the treatment of chronic pain: a 20 year literature review. J Neurosurg 2004;100:254-67.

167. Turner JA, Loeser JD, Deyo RA, Sanders SB. Spinal cord stimulation for patients with failed back surgery syndrome or complex regional pain syndrome: a systematic review of effectiveness and complications. Pain 2004;108:137-47. 
168. Kumar AJ, Buchser E, Linderoth B, Meglio M, Van Buyten J. Avoiding Complications From Spinal Cord Stimulation: Practical Recommendations From an International Panel of Experts. Neuromodulation 2007;10:26-33.

169. Mogil JS. Animal models of pain: progress and challenges. Nat Rev Neurosci 2009;10:283-94.

170. Yezierski RP, Vierck CJ. Reflex and pain behaviors are not equivalent: lessons from spinal cord injury. Pain;151:569-70.

171. de Boer RD, Marinus J, van Hilten JJ, Huygen FJ, van Eijs F, van Kleef M, Bauer MC, van Gestel M, Zuurmond WW, Perez RS. Distribution of signs and symptoms of Complex Regional Pain Syndrome type I in patients meeting the diagnostic criteria of the International Association for the Study of Pain. Eur J Pain;15:830 e1-8.

172. Marinus J, Moseley GL, Birklein F, Baron R, Maihofner C, Kingery WS, van Hilten JJ. Clinical features and pathophysiology of complex regional pain syndrome. Lancet Neurol;10:637-48.

173. Alexander GM, van Rijn MA, van Hilten JJ, Perreault MJ, Schwartzman RJ. Changes in cerebrospinal fluid levels of pro-inflammatory cytokines in CRPS. Pain 2005;116:213-9.

174. Li L, Xian CJ, Zhong JH, Zhou XF. Effect of lumbar 5 ventral root transection on pain behaviors: a novel rat model for neuropathic pain without axotomy of primary sensory neurons. Exp Neurol 2002;175:23-34.

175. Chaplan SR, Bach FW, Pogrel JW, Chung JM, Yaksh TL. Quantitative assessment of tactile allodynia in the rat paw. J Neurosci Methods 1994;53:55-63.

176. Guo TZ, Wei T, Kingery WS. Glucocorticoid inhibition of vascular abnormalities in a tibia fracture rat model of complex regional pain syndrome type I. Pain 2006;121:158-67.

177. Gradl G, Finke B, Schattner S, Gierer P, Mittlmeier T, Vollmar B. Continuous intra-arterial application of substance $P$ induces signs and symptoms of experimental complex regional pain syndrome (CRPS) such as edema, inflammation and mechanical pain but no thermal pain. Neuroscience 2007;148:757-65.

178. Mailis A, Wade J. Profile of Caucasian women with possible genetic predisposition to reflex sympathetic dystrophy: a pilot study. Clin J Pain 1994;10:210-7.

179. Cutler G, Marshall LA, Chin N, Baribault H, Kassner PD. Significant gene content variation characterizes the genomes of inbred mouse strains. Genome Res 2007;17:1743-54.

180. Oliver PL, Bitoun E, Davies KE. Comparative genetic analysis: the utility of mouse genetic systems for studying human monogenic disease. Mamm Genome 2007;18:412-24.

181. Mogil JS, Wilson SG, Bon K, Lee SE, Chung K, Raber P, Pieper JO, Hain HS, Belknap JK, Hubert L, Elmer GI, Chung JM, Devor M. Heritability of nociception I: responses of 11 inbred mouse strains on 12 measures of nociception. Pain 1999;80:67-82.

182. DeLeo JA, Rutkowski MD. Gender differences in rat neuropathic pain sensitivity is dependent on strain. Neurosci Lett 2000;282:197-9.

183. Nissenbaum J, Devor M, Seltzer Z, Gebauer M, Michaelis M, Tal M, Dorfman R, Abitbul-Yarkoni M, Lu Y, Elahipanah T, delCanho S, Minert A, Fried K, Persson AK, Shpigler H, Shabo E, Yakir B, Pisante A, Darvasi A. Susceptibility to chronic pain following nerve injury is genetically affected by CACNG2. Genome Res;20:1180-90.

184. Moore KA, Kohno T, Karchewski LA, Scholz J, Baba H, Woolf CJ. Partial peripheral nerve injury promotes a selective loss of GABAergic inhibition in the superficial dorsal horn of the spinal cord. J Neurosci 2002;22:6724-31.

185. Gazelius B, Cui JG, Svensson M, Meyerson B, Linderoth B. Photochemically induced ischaemic lesion of the rat sciatic nerve. A novel method providing high incidence of mononeuropathy. Neuroreport 1996;7:2619-23.

186. Castro-Lopes JM, Coimbra A, Grant G, Arvidsson J. Ultrastructural changes of the central scalloped (C1) primary afferent endings of synaptic glomeruli in the substantia gelatinosa Rolandi of the rat after peripheral neurotomy. J Neurocytol 1990;19:329-37. 
187. Ibuki T, Hama AT, Wang XT, Pappas GD, Sagen J. Loss of GABA-immunoreactivity in the spinal dorsal horn of rats with peripheral nerve injury and promotion of recovery by adrenal medullary grafts. Neuroscience 1997;76:845-58.

188. Schechtmann G, Lind G, Winter J, Meyerson BA, Linderoth B. Intrathecal clonidine and baclofen enhance the pain-relieving effect of spinal cord stimulation: a comparative placebo-controlled, randomized trial. Neurosurgery;67:173-81.

189. Lind G, Schechtmann G, Winter J, Linderoth B. Drug-enhanced spinal stimulation for pain: a new strategy. Acta Neurochir Suppl 2007;97:57-63.

190. Saade NE, Tabet MS, Soueidan SA, Bitar M, Atweh SF, Jabbur SJ. Supraspinal modulation of nociception in awake rats by stimulation of the dorsal column nuclei. Brain Res 1986;369:307-10.

191. Campbell JN. Examination of possible mechanisms by which stimulation of the spinal cord in man relieves pain. Appl Neurophysiol 1981;44:181-6.

192. Kiefer RT, Rohr P, Ploppa A, Dieterich HJ, Grothusen J, Koffler S, Altemeyer KH, Unertl K, Schwartzman RJ. Efficacy of ketamine in anesthetic dosage for the treatment of refractory complex regional pain syndrome: an open-label phase II study. Pain Med 2008;9:1173-201.

193. Mei X, Wang W, Li Y, Zhang H, Wu S, Xu L. Inhibiting astrocytic activation: a novel analgesic mechanism of ketamine at the spinal level? J Neurochem 2009;109:1691-700.

194. Janssen SPM, Gerard S, Truin M, Van Kleef M, Joosten EAJ,. Spinal cord stimulation as a painrelieving therapy in experimental chronic neuropathy attenuates intracellular GABA levels article under review 2011.

195. Gao W, Chen G, Reinert KC, Ebner TJ. Cerebellar cortical molecular layer inhibition is organized in parasagittal zones. J Neurosci 2006;26:8377-87.

196. Shibuki K, Hishida R, Murakami H, Kudoh M, Kawaguchi T, Watanabe M, Watanabe S, Kouuchi T, Tanaka R. Dynamic imaging of somatosensory cortical activity in the rat visualized by flavoprotein autofluorescence. J Physiol 2003;549:919-27.

197. Jongen JL, Pederzani T, Koekkoek SK, Shapiro J, van der Burg J, De Zeeuw Cl, Huygen FJ, Holstege JC. Autofluorescent flavoprotein imaging of spinal nociceptive activity. J Neurosci;30:4081-7.

198. Jongen JL. abstract S-3942, 2011.

199. Perez RS, Collins S, Marinus J, Zuurmond WW, de Lange JJ. Diagnostic criteria for CRPS I: differences between patient profiles using three different diagnostic sets. Eur J Pain 2007;11:895-902.

200. Eberle T, Doganci B, Kramer HH, Geber C, Fechir M, Magerl W, Birklein F. Warm and cold complex regional pain syndromes: differences beyond skin temperature? Neurology 2009;72:505-12. 
Slotoverweging en dankwoord 

Eindelijk, na zeven jaar is het dan zover; opleiding klaar, proefschrift klaar, de eerste anesthesie AGIKO (survivor) van Maastricht is afgeleverd. Een project van deze omvang vereist veel werk, vrije tijd en niet te vergeten offers van het thuisfront (als ik weer eens dagen achter de laptop zat of eigenlijk alleen even thuis kwam om te slapen). Hard werken is echter nooit erg zolang de sfeer goed is en er ook goed gelachen kan worden. Een zeer belangrijk aspect van promoveren is dat het zeker geen 'one man show' is. Je bent permanent afhankelijk van de expertise en hulp van anderen. Dit varieert van het maken en op tijd leveren van een failure proof SCS electrode door technici van Medtronic tot de juiste levering van proefdieren, correctie van teksten of het binnenhalen van onderzoeksgelden. Vandaar dat dit zeer belangrijke hoofdstuk zeker niet in het proefschrift mag ontbreken; een woord van dank voor allen die op enige wijze aan dit proefschrift hebben bij gedragen. De promotie en opleiding zijn altijd zeer sterk met elkaar verweven geweest. Gezien de simultane afronding van beiden is dit dankwoord dan tevens een gepast afscheidswoord aan allen met wie ik zeven jaar lang in het AZM heb samen gewerkt.

Prof. Dr. Van Kleef mijn promotor. Het probleem 'Smits' (een van die mappen in de kast rechts naast uw bureau) is nu dan toch opgelost. Ik herinner me nog dat u op mijn sollicitatie gesprek het ijs brak toen er door de vertegenwoordigster van de assistenten werd gesteld dat ik nogal solitaire hobby's beoefende zoals gitaarspelen. Waarop u reactie was: 'Moet die jongen dan aan line-dancing gaan doen om hier te mogen komen werken,?' Ik heb gelukkig later ook nog aangetoond dat je gitaarspelen helemaal niet solitair hoeft te doen, de eerste CD die uitkomt is overigens voor u. Bedankt in ieder geval voor de geboden hulp, steun en vertrouwen. Gedurende de afgelopen jaren er was altijd; toegankelijkheid, interesse, (even binnenlopen bij een nieuw experiment) een luisterend oor, en op zijn tijd de nodige goede raad. (en soms ook een gratis notitieblok om dingen op te schrijven). Bedankt !

Dr. Bert Joosten, co-promotor, ik weet nog goed de begintijd op het lab, Ronnie, Guido en Helwin, alles overzichtelijk, 3 brave jongetjes. Maar het lab groeide en groeide, stapels te corrigeren tekst op je bureau, nieuwe AGIKO's, AIO's en studenten van een divers pluimage. Toch bleef je dezelfde rustige en prettige persoonlijkheid om mee te werken. Jij kent de AGIKO's door en door en weet hoe met ons om te gaan. Je hebt bij mij en de andere AGIKO's in ieder geval groot respect geoogst als een zeer slimme wetenschapper en als mens. Ik heb heel veel dingen van je geleerd. Niet onbelangrijk is dat je ook een aanjager bent van een goede sfeer op het lab. Even met z'n allen lunchen in de MPR, een barbecue in 'Heitze' of 'de resepsie van prins Ber den ierste' (waarna Ronnie nog even een overstekende ree schepte met zijn BMW) 
Dr. Marius Kemler co-promoter, bedankt voor je expertise en kritische inbreng bij het klinische stuk. Ik realiseerde me pas dat ik alleen artikelen van jouw hand heb gelezen maar je eigenlijk nog nooit in het echt heb gezien.

De voorzitter en andere leden van de beoordelings commissie van mijn proefschrift (zie blz 4 van dit proefschrift) Voor het op zo'n korte termijn doornemen van mijn proefschrift. Zonder jullie hulp had een promotie in december er niet ingezeten.

Prof M. Marcus. Hij kent Helwin na zeven jaar echt wel door en door. Als opleider had je een heel goed begrip van de AGIKO constructie en je wist vaak ook goed wat er privé bij de mensen afspeelde (nee, ik ben niet zwanger). Regelmatig hadden we levendige discussies over allerlei zaken en vaak had je dan achteraf (bijna) altijd gelijk. Je geweldige (zwarte) humor kon bij mij de meest grijze dag tot een succes maken als we weer eens dienst hadden. Ik heb veel van je geleerd en ken je als een opleider die pal achter zijn assistenten staat. Bedankt voor alles.

Dr. Jan Van Zundert, je bent al vanaf het begin van mijn promotie project aanwezig waar nodig altijd bereid geweest tot meedenken, meehelpen of het geven van advies. Ik zie er naar uit om met jullie te mogen werken en mij verder te mogen ontwikkelen in Genk.

Dr. R. Heylen, bedankt voor de mogelijkheid om in Genk te mogen komen werken en de pijnopleiding te volgen.

Dr. J. Patijn, bedankt voor meedenken en advies in dit project.

Prof H. Steinbusch, bedankt dat ik te gast mocht zijn op uw afdeling voor het doen van experimenteel werk. Steeds weer toonde u ook persoonlijke interesse in het SCS project en gaf waar nodig tips.

Keith Mullet from Medtronic. Thanks for your support to this project. Your intelligent remarks on scientific matters were always quite useful. The neuroscience fairs in Switzerland were legendary and did always create scientific momentum in the field of experimental neurostimulation. Your help was crucial to this project and it was a pleasure to work with you. Lots of luck in France.

Paul and victor from Medtronic. (Re)- designing and producing a reliable standardized rat and mouse SCS electrode system for was crucial to many experimental SCS projects. The word impossible does not exist in your vocabulary and the electrodes and other devices were always delivered in time for our experiments. This, combined with a good sense of humor is a success in any business. I suspect that Victor 
was actually locked up in his worshop for days by Paul to produce and weld the enormous amounts of anodes and cathodes that had to be delivered. Paul's technical drawings are amazing (see the cover of this thesis) and he just shakes them out of his wrist in seconds.

De leiding en alle andere medewerkers van het TREND consortium (teveel mensen om op te noemen). Voor hulp en steun voor mijn promotie project. Ik ben zeer onder de indruk en trots op de enorme wetenschappelijke output van TREND en ben blij hiervan deel te mogen uitmaken.

Prof. Dr. Frank Huygen en Dr. Joost jongen, bedankt voor jullie fijne samenwerking met het AFI project. De ivoren toren in Rotterdam voelt voor mij nog altijd een beetje als thuis.

Fons Kessels, bedankt voor je hulp met het de database en het schrijven van het klinische artikel, ik vond het een zeer goede en ook leuke samenwerking.

Jose Geurts, bedankt voor je SPSS kunsten, het managen van de patiënten database en het meedenken en schrijven van het klinische artikel.

Wiel Honig. Als er weer eens iets met je computer aan de hand is hoop je altijd maar weer dat ie er is en ook in het werken met proefdieren is er geen betere te vinden. Als je denkt dat je weet wat honger is moet je eens met Wiel een dagje door de sneeuw gaan wandelen in Stockholm en daarna naar de Bronco's Bar gaan om daar een Bronco Burger 2 in luttele seconden te zien verdwijnen. Je creativiteit met de diertondeuse is ongekend, maar toegegeven je wenkbrauwen zijn altijd perfect. Je bent me d'r een, maar wel een goeie, bedankt voor al je hulp.

Bengt Linderoth and Bjorn Meyerson. Thanks for your hospitality and for giving us an opportunity to learn the techniques of experimental SCS in your lab at the Karolinska Institute in Stockholm back in 2005. It was the spark that started this project.

Camilla Ultenius. Thanks for the wonderful cooperation in 2005 and 2006 that led to the publications of the first article of my thesis.

Ronald Deumens, vaak heb je me met allerlei zaken geholpen, met je ruime ervaring in het lab was in je onmisbaar voor de dokters uit de kliniek die dier experimenteel werk kwamen doen. Je stond altijd voor ons klaar. We hebben samen veel gelachen altijd ging 't weer over...eh... Een ding begrijp ik nog steeds niet: waarom heb je in hemelsnaam destijds die ree niet in de kofferbak geladen. Een vriezer vol vlees was 
toch een redelijke compensatie voor je kapotte auto en voor een stukje rug had ik geholpen om hem schoon te maken.

Alle stafleden anesthesiologie van het MUMC+. Voor alles wat ik van jullie heb geleerd op het gebied van anesthesie en alle gezellige en ontspannen momenten die er ook vaak waren. In het bijzonder wil ik mijn mentoren Henriette willigers en Frank Kwinten bedanken voor hun immer beschikbare goede raad en daad. Ook Bas Timmerman die in feite een officieuze mentor en vriend is wil ik bedanken voor je adviezen in zake anesthesiologische problemen maar ook vaak gewoon de gezellige gesprekken en sfeer op de OK (en bedankt nog voor het prachtige boek).

De dames van het secretariaat anesthesie en in het bijzonder Patrice die altijd weer een wakend oog hield mbt opleidingszaken en het invullen van noodzakelijke formulieren, inplannen van vakantiedagen, etc..etc.. Bedankt.

De mede assistenten van de afdeling anesthesiologie, het was fijn en gezellig om met jullie samen te werken.

Het personeel van de recovery. Als jullie je afvroegen wat ik vaak nog zo laat in het computerhok aan het doen was. Zie hier mijn proefschrift, het gaat jullie goed, (ook diegene met de bezem) bedankt voor de fijne samenwerking.

Ingrid Mus, pijnzuster, je bent altijd wel een beetje mijn grote vriendin geweest in die zeven jaar, bedankt voor je gezellige samenwerking.

Alle anesthesiemedewerkers in het AZM. Bij rustige OK's zorgden jullie ervoor dat ik ongestoord door kon schrijven zodat alles op tijd is afgekomen bedankt voor jullie samenwerking, steun en gezelligheid.

Michiel Truin, je hebt SCS in de muis klaargespeeld dat was zeker geen gemakkelijke opgave. Inderdaad hebben we ook veel gelachen als er weer eens een hilarische 'photo shop job' van jouw hand circuleerde. In onze kamer hadden we zo onze eigen dingetjes die niemand verder echt begreep. Ik hoop overigens dat je inmiddels geen financiële steun meer verleent aan de firma Philip Morris en wens je veel succes in je vervolg opleiding.

Wouter Pluijms. Als SCS mannen hebben we elkaar vaak wel wat te vertellen. Jouw affiniteit met statistiek is voor de omgeving soms erg handig. Je hebt nog een aardige opleidingsweg te gaan maar de uitkomst zal zeker goed zijn. Leer nog wel een beetje meer $\mathrm{BHBH}$. Veel succes met alles. 
Sophie Janssen, We hadden vaak discussie over het Seltzer model en de uitkomsten hiervan. Gelukkig hebben we ook nog goed gelachen om onzin. Bedankt voor je samenwerking.

Robbie en Liesbeth, jullie werk en aanwezigheid aan de UM is zeer waardevol en kenmerkt in mijn optiek de beginfase van een daadwerkelijke Europese samenwerking waarbij de huidige grenzen zullen vervagen. Bedankt voor jullie hulp en gezelligheid.

Job Gerver, bedankt voor jouw hulp bij het c-Fos project. Jou succesverhaal van chronisch uitgeloot (had ik ook) worden voor geneeskunde en dan via een selectie traject toch binnenkomen en dan via de versnelde opleiding nu al klaar is een topprestatie die de waanzinnigheid van het lotingsysteem benadrukt.

Alle andere mensen van het NP lab die zorgden voor hulp, advies en goede sfeer, , een goede mop, of een geweldig youtube filmpje, bedankt hiervoor.

De Medewerkers van assurantiën kantoor en regiobank Helwegen. Ik heb het kantoor vaak gebruikt als werkplek, bedankt voor jullie gezelligheid en hulp.

Dr. Djo Hassan (neuroloog-intensivist). Een goede vriend sinds 1995 bij wie ik als student al met wetenschap in aanraking kwam. We hebben veel gelachen (soms ook om een dode mus) en Ik heb veel van je geleerd op afd 6 zuid IC in het Erasmus MC. Ook in de laatste fase van het proefschrift was je weer bereid mee te denken, bedankt.

Geert en Lynyrd, bedankt voor de samenwerking in de muziek. Ik heb nog altijd het sterke gevoel dat we een hit gaan scoren (en afschuwelijk rijk worden) als we de boel nu eindelijk eens een keer gaan opnemen.

De firma Douwe Egberts voor hun erg speciale koffie uit de automaat op het lab die waarschijnlijk deel uitmaakt van het grootste LD50 experiment dat ooit op een $\mathrm{Ne}$ derlandse universiteit is uitgevoerd.

$\mathrm{Pa}$ en $\mathrm{Ma}$ en Alinde, jullie hebben altijd geholpen in voor en tegenspoed. $\mathrm{Pa}$, we hebben zelfs nog eventjes een sauna gebouwd in de tussentijd. Ik hoop dat jullie nog lang mogen genieten in gezondheid.

Pierre en Gerry Helwegen 2007 , altijd jullie hulp met raad en daad. 
Pierre, altijd vlaai in de koelkast, een gezellige buurman, schoonvader en opa die er altijd is, bedankt voor al jouw hulp en steun, ondanks de moeilijke tijd met het overlijden van je vrouw. We hopen nog lang naast je te wonen.

Mia, mijn (mooie, lieve en charmante) vrouw die steeds weer voor eten, kinderen, huishouden en god mag weten wat allemaal nog meer zorgde als ik weer eens laat of niet thuis was. Zonder jouw hulp had ik dit proefschrift niet klaar gekregen.

Pierre en Neele, mijn lieve en slimme kindertjes pappa zet nu een streep onder dit boekje en is blij om meer tijd met jullie te mogen doorbrengen. 


\section{Curriculum Vitae}

Helwin Smits werd op 29 juni 1972 geboren te Strijen, in het prachtige polderlandschap van de Hoeksche Waard. Na een onbezorgde jeugd volgde de middelbare schooltijd op de Prinses Juliana Scholengemeenschap en het Christelijk Lyceum te Dordrecht. Na het VWO examen in 1992 werd hij uitgeloot voor de studie geneeskunde en maakte hij gedurende 12 maanden deel uit van een Leopard 2 tankbemanning als dienstplichtig militair bij het 101 tankbataljon te Soesterberg. In 1994 starte hij met de studie geneeskunde aan de Erasmus Universiteit te Rotterdam en als student werkte hij mee aan een wetenschappelijk project op de afdeling Experimentele Anesthesiologie bij professor B. Lachmann. In 2001 werd het arts examen behaald. Tijdens zijn werk als arts-assistent Intensive -Care (2002-2004) in het Viecurie Medisch Centrum te Venlo werd Helwin's interesse voor de anesthesiologie verder aangewakkerd en eind 2004 startte hij met de opleiding tot anesthesioloog in het AZM in combinatie met een promotie onderzoek (AGIKO) die in oktober 2011 werd afgerond. 